\title{
Melhor Coaproximação em Espaços Normados
}

\author{
Jorge Ariel Brisset Páez
}

\author{
DISSERTAÇÃO APRESENTADA \\ $\mathrm{AO}$ \\ INSTITUTO DE MATEMÁTICA E ESTATÍSTICA \\ DA \\ UNIVERSIDADE DE SÃO PAULO \\ PARA \\ OBTENÇÃO DO GRAU DE MESTRE \\ EM \\ MATEMÁTICA
}

\begin{abstract}
Área de Concentração: Análise Funcional Orientadora: Profa. Dra. Carmen Silvia Cardassi
\end{abstract}

Durante a elaboração deste trabalho o autor recebeu apoio financeiro do CAPES

-São Paulo, abril de 1999- 


\title{
Melhor Coaproximação em Espaços Normados
}

\author{
Este exemplar corresponde à redação \\ final da dissertação devidamente \\ corrigida e defendida por Jorge Ariel Brisset \\ e aprovada pela comissão julgadora.
}

São Paulo, abril de 1999.

Banca examinadora:

- Profa. Dra. Carmen Silvia Cardassi (Orientadora) - IME-USP

- Prof. Dr. Chaim Samuel Hönig - IME-USP

- Prof. Dr. Raymundo Luiz de Alencar - IMECC-UNICAMP 


\section{RESUMO}

Em 1972, Franchetti e Furi [F-F72] introduziram um tipo de aproximação em espaços normados chamado de melhor coaproximação. Este trabalho estuda a existência e a unicidade de melhor coaproximação em subconjuntos convexos, algumas caracterizações para espaços de Hilbert e uma caracterização para espaços estritamente convexos. Também veremos a melhor coaproximação polinômica cle uma função contínua e propriedades do operador melhor coaproximação.

\footnotetext{
ABSTRACT

In 1972, Franchetti and Furi [F-F72] introduced a kind of approximation in normed spaces, called best coapproximation. This work is concerned with the existence and uniqueness of best coapproximation by elements of convex subsets, characterizations of elements of best coapproximation, some characterizations of Hilbert spaces and a characterization of strictly convex spaces. Best polynomial coapproximation of continuous functions and properties of the best coapproximation operator are also presented.
} 
a mi Martinacho. 


\section{Agradecimentos I:}

- Antes que nada, quiero agradecer a los que tienen la paciencia de leer tantos agradecimientos.

- A mi hijo, Martín: por seguir tan unido a mí como el primer día.

- A mi querida familia: por haberme apoyado siempre y aguantarme cada vez que fui.

- A mis padres, Celso y Elena (no hace falta decir porqué).

- A mis hermanos, Miguel, Susy y Tito: por el apoyo.

- A mis sobrinos, (in alphabetical order) Andrea, Claudia, Danielo, Dieguito, Fede, Fernando, Naty, Nico y Rodri: por hacerme pensar en ellos y extrañarlos tanto.

- A mis tías Mirza y Soñita: por ser pésimas jugadoras de "tuti cabrero" y de "rummy canasta" .

- A mi amor, Lety Pery: por su incondicional amor y porque "... no es perfecta mas, se acerca a lo que yo simplemente soñé..."

- A la familia de Leticia, sin excepción, por algo muy simple y enorme: dejarme ser uno de ellos.

- A mi mejor amigo, Danielo: simplemente por eso.

- Al "drink-team" del Liceo 13: por asegurarme el puesto de golero y por haber realizado juntos tantos "brindis por pierrot" en el "Renta".

- A mis compañeros de los liceos 13 "Ytuzaingó" y "El Clemente": por el estímulo y por recibirme siempre con los brazos abiertos.

- A todos aquellos que debí mencionar aquí y me olvidé: por no enojarse ante tal omisión.

- A la justicia española: por haberme hecho creer que la justicia tarda pero llega.

- Al campeón del siglo, el CLUB ATLÉTICO PEÑAROL: por darme tantas alegrías. 


\section{Agradecimentos II:}

- A Carmen: por su excelente orientación y por ser tan buena profesora y persona.

- A los profesores Chaim, Elza, Enrique, Lúcia, Débora, Roseli, Carmen y Albo: por sus enseñanzas tanto matemáticas como didácticas.

- A los profesores Futorny y Menshikov: por su invalorable ayuda en las traducciones de ruso y sus "tours" por la biblioteca.

- A los profesores uruguayos Borghi, Barreiro y Mara: porque gracias a ellos aprendí a querer la matemática.

- Al profesor Alfredo Guido: por haber sido quien más influyó en mí cuando decidí hacer la maestría.

- A Marina: por haber conseguido el material de Rusia.

- A mis amigos, Cecilia, Irene, José, Jocirei, Ramiro, Ronaldo, Marcelo, Fernando, Daniela y Marcos: por haber hecho de nuestras casas un hogar (además de haber "arreglado el mundo" varias veces con un mate en la mano).

- A mis vecinos, Maria, José. Marina, Emivan, Adriana, Luciana, Marcelo, Maria do Carmo, Lenir, Edna, Andrea, Jeferson y Adriana: por haberme aguantado a mí y a mis amigos y no protestar por estar tan alta la música.

- A Héctor y Marcela Merklen: por todas las "gauchadas" que me hicieron.

- A mis hermanos brasileños, Bárbara, Regina. Daniel, Liane, José, Sidney, Marco Aurélio, Samuel, Raul, Claus, Fernando, Jocirei, Ronaldo, Mário, Edson, Edson, Rolo. Caputi, Paulo. Ayumi, Cintia, Márcio, Juaci, Jair, Gláucio, Lúcia. Marcelo, Calixto, Walter, Daniela, Clézio, Leandro, Alice, Saidi, Kao. Kennedy, Branderberger, Douglas, Maria, Walquíria, Marcelo, Loreley, Vanderlei, Lucia : por permitir que me integrara a ellos como uno más. 
- A mis hermanos hispanoamericanos y también de otras latitudes, Germán, Santos, Nelson, Xyoby, Nestor. Alberto, Antonio, Gaspar, Carlos, Cristian, Mariano, Carlos, Laura. Laura, Gonzalo, Lucho. Raúl, Juan, Ivo, Ricardo, Gladys, Sandra. Paola, Jairo, José, Hernán, Irene, Carolina, Marcel, Claudia, Milton. Pablo. Juan Carlos : por los tan lindos momentos que pasamos juntos y por el riquísimo intercambio cultural, el cual no fue justo, pues recibí mucho más de lo que di. 


\section{Sumário}

Introdução 1

1 Melhor Coaproximação: Definições e Exemplos 3

1.1 Definiçōes e Proposiçōes Básicas . . . . . . . . . . . . . . . 3

1.2 Exemplos ........................... 5

2 Existência e Unicidade da Melhor Coaproximação $\quad 15$

2.1 Ortogonalidade. . . . . . . . . . . . . . . . . . 15

2.2 Existência da Melhor Coaproximação. . . . . . . . . . . . . . . 27

2.3 Funções Convexas e Diferenciabilidade. . . . . . . . . . . . . . . . 37

2.4 Unicidade e Linearidade da Melhor Coaproximação. . . . . . . . . . . 43

3 Hipercírculos de Chebyshev e Coaproximação $\quad 47$

3.1 Hipercírculos e Centros de Chebyshev . . . . . . . . . . . . . . . 47

3.2 Uma Caracterização para Espaços de Hilbert . . . . . . . . . . . . . 58

4 Caracterizações de Melhor Coaproximação e Aplicações $\quad 64$

4.1 Caracterizações de Melhor Coaproximação . . . . . . . . . . . . . . 65

4.2 Melhor Coaproximação Polinômica . . . . . . . . . . . . . . 73

4.3 Uma Caracterização para Espaços Estritamente Convexos . . . . . . 80

5 Propriedades do Operador $R_{C} \quad 86$

5.1 Propriedades de $R_{G} \ldots \ldots \ldots \ldots \ldots$. . . . . . . . . . . . . . . . . . . .

5.2 Caracterizações de espaços de Hilbert . . . . . . . . . . . . . . . . . . . . . 94

5.3 Propriedades de $R_{C} \ldots \ldots \ldots \ldots$. . . . . . . . . . . . . . . . 101

$5.4 R_{C}$ e a retração ortogonal . . . . . . . . . . . . . . . 104

5.5 Aproximação, coaproximação e retraçào ortogonal fortes . . . . . . 113

$\begin{array}{lr}\text { Referências Bibliográficas } & 122\end{array}$ 


\section{Introdução}

Em 1972 C. Franchetti e M. Furi [F-Fr2] introduziram uma nova noção de aproximação em espaços normados, hoje conhecida como melhor coaproximação.

A partir daí, diversos matemáticos estudaram o assunto, entre os quais P. L. Papini e I. Singer.

Em 1991, T. D. Narang escreveu um survey [Nar91] da matéria entitulado:

On best coapproximation in normed linear spaces, Rocky Moun. J. Math. 22 (1991), $265-287$.

O conceito de melhor coaproximação está fortemente vinculado com a ortogonalidade no sentido da definição de G.Birkhoff [Bir35]. Esta ortogonalidade não é a única estudada em espaços normados quaisquer. Por exemplo, em [Jam45] podemos encontrar, além dessa, a isósceles e a pitagórica. A ortogonalidade de Birkhoff está também vinculada com a melhor aproximação e será utilizada em respectivas caracterizações.

Este trabalho pretende introduzir o conceito de melhor coaproximação e compará-lo com outros tipos de aproximação, como a melhor aproximação e a retração ortogonal ([Bru73]), estudar relações com outros tópicos e achar propriedades da melhor coaproximação, no caso em que possa ser visto como operador.

Apresentamos diversos exemplos. Nos primeiros utilizaremos basicamente a definição, visando a melhor compreensão do novo conceito; depois usamos, em algums casos particulares, os resultados teóricos obtidos no decorrer do trabalho. 
Quase todas as provas estào incluidas no texto. A exclusão de algumas provas é devida, fundamentalmente, à extensão do trabalho. Nestes casos serão dadas as referências bibliograficas onde o leitor pode achá-las.

No capítulo 1, apresentamos o conceito de melhor coaproximação estudando varios exemplos [Dav63]. Também veremos alguns resultados básicos do assunto mas de uma grande utilidade em todo o trabalho.

No capítulo 2, estudaremos condições para a existência e para a unicidade da melhor coaproximação. Para isso. veremos previamente alguns resultados sobre ortogonalidade, funções convexas e diferenciabilidade de Gâteaux ([Jam45], [Jam47a], [Phe89], [Hol72], [Jam47b], [Kak39]).

No capítulo 3, veremos uma caracterização para espaços de Hilbert a partir dos espaços reflexivos, estudando previamente algumas propriedades de hipercírculos e centros de Chebyshev [Fra75].

No capítulo 4, obteremos caracterizações da melhor coaproximação a partir de outras já conhecidas de melhor aproximação. Como aplicação, estudaremos em $X=\left(C[0,1],\|\|_{\infty}\right)$ a melhor coaproximação tanto polinômica como em subespaços de polinômios e uma caracterização para espaços estritamente convexos, muito parecida a uma conhecida que utiliza a melhor aproximação [Sin70]).

No capítulo 5, estudaremos propriedades do operador melhor coaproximação sobre subespaços, que vão nos permitir obter outras caracterizações para espaços de Hilbert ([Papi4], [Papij]), e sobre subconjuntos convexos ([Papi6]). Neste último aspecto, estudaremos também o conceito de melhor coaproximação forte, comparando os resultados com os da melhor aproximação e da retração ortogonal. A finalidade é que o trabalho tenha um capítulo. no qual o leitor possa achar todas as propriedades do operador coaproximação, embora algumas delas tenham sido introduzidas ao estudar outros aspectos da melhor coaproximação.

Em toda a dissertação, trabalharemos com espaços normados reais. 


\section{Capítulo 1}

\section{Melhor Coaproximação: Definições e Exemplos}

O objetivo principal deste capítulo é introduzir o conceito de melhor coaproximação. Além disso, às vezes faremos comparações com a conhecida melhor aproximação. Trabalharemos alguns exemplos que servem para esclarecer o conceito de melhor coaproximação. Também provaremos neste capítulo proposições básicas do assunto, mas de uma grande utilidade para todo o trabalho.

\subsection{Definições e Proposições Básicas}

Definição 1.1.1. Dados $X$ um espaço normado, $x \in X$ e $C$ um subconjunto de $X$, dizemos que $g_{0} \in C$ é ponto de melhor aproximação de $x$ em $C$ se para todo $g$ em $C$ vale a seguinte desigualdade

$$
\left\|x-g_{0}\right\| \leq\|x-g\|
$$

O conjunto dos pontos $g_{0}$ que satisfazem (1.1) será representado por $P_{C}(x)$

Definição 1.1.2. Dados $X$ um espaço normado, $x \in X$ e $C$ um subconjunto de $X$, dizemos que $g_{0} \in C$ é ponto de melhor coaproximação de $x$ em $C$ se para todo $g$ em $C$ vale a seguinte desigualdade 


$$
\left\|g_{0}-g\right\| \leq\|x-g\| .
$$

O conjunto dos pontos $g_{0}$ que satisfazem (1.2) será representado por $R_{C}(x)$

Proposição 1.1.3. Sejam X um espaço normado. C um subconjunto de $X$ e $x$ um ponto de $X$.

Então $P_{C}(x)=C \cap A_{C}(x)$, onde

$$
A_{C}(x)=\bigcap_{g \in C} \bar{B}_{\|x-g\|}(x) .
$$

\section{Prova :}

Para $g_{0} \in P_{C}(x)$, pela definição 1.1.1 temos que $g_{0} \in C$. Além disso, $g_{0}$ satisfaz (1.1) e então temos que $g_{0} \in \bar{B}_{\|x-g\|}(x)$ para todo $g \in G$. Portanto, $g_{0} \in A_{C}(x)$.

Reciprocamente, se $g_{0} \in C \cap A_{C}(x)$, temos que $g_{0} \in C$ e

$$
\left\|x-g_{0}\right\| \leq\|x-g\| \text { para todo } g \in C .
$$

Proposição 1.1.4. Sejam X um espaço normado. $C$ um subconjunto de $X$ e $x$ um ponto de $X$.

Entĩo $R_{C}(x)=C \cap B_{C}(x)$, onde

$$
B_{C}(x)=\bigcap_{g \in C} \bar{B}_{\|x-g\|}(g)
$$

\section{Prova :}

Análoga à proposição anterior.

Observação 1.1.5. Da proposição 1.1 .4 deduz-se que o conjunto $R_{C}(x)$ é limitado por $\|x\|$ se $0 \in C$ (por exemplo se for subespaço) e apenas limitado se for um subconjunto $C$ qualquer. Além disso. será fechado e convexo se $C$ também o for.

Na próxima seção veremos alguns exemplos utilizando as proposições 1.1.3 e 1.1.4. 


\subsection{Exemplos}

Exemplo 1.2.1. Consideremos o plano $\mathbb{R}^{2}$ com a norma \|\|$_{2}$. Sejam $x=(0,1)$ e $C$ o eixo $x_{1}$. É fácil ver que neste caso os conjuntos de melhor aproximação e de melhor coaproximação são iguais:

$$
P_{C}(x)=R_{C}(x)=\{(0,0)\}
$$
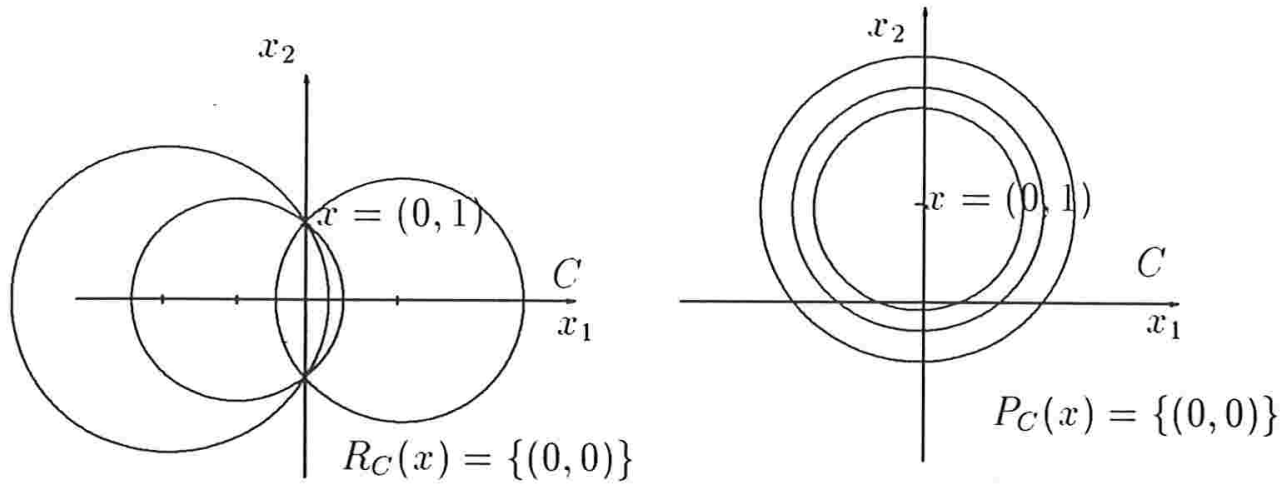

Figura 1.1.

Exemplo 1.2.2. Consideremos novamente o plano $\mathbb{R}^{2}$ com a norma \|\|$_{2}$.

Sejam $x=(3,0)$ e $C$ a bola fechada $\bar{B}_{1}(0)$. Neste caso os conjuntos de melhor aproximação e de melhor coaproximação não são iguais, pois

$$
P_{C}(x)=\{(1,0)\} \text { e } R_{C}(x)=\bar{B}_{1}(0)=C .
$$
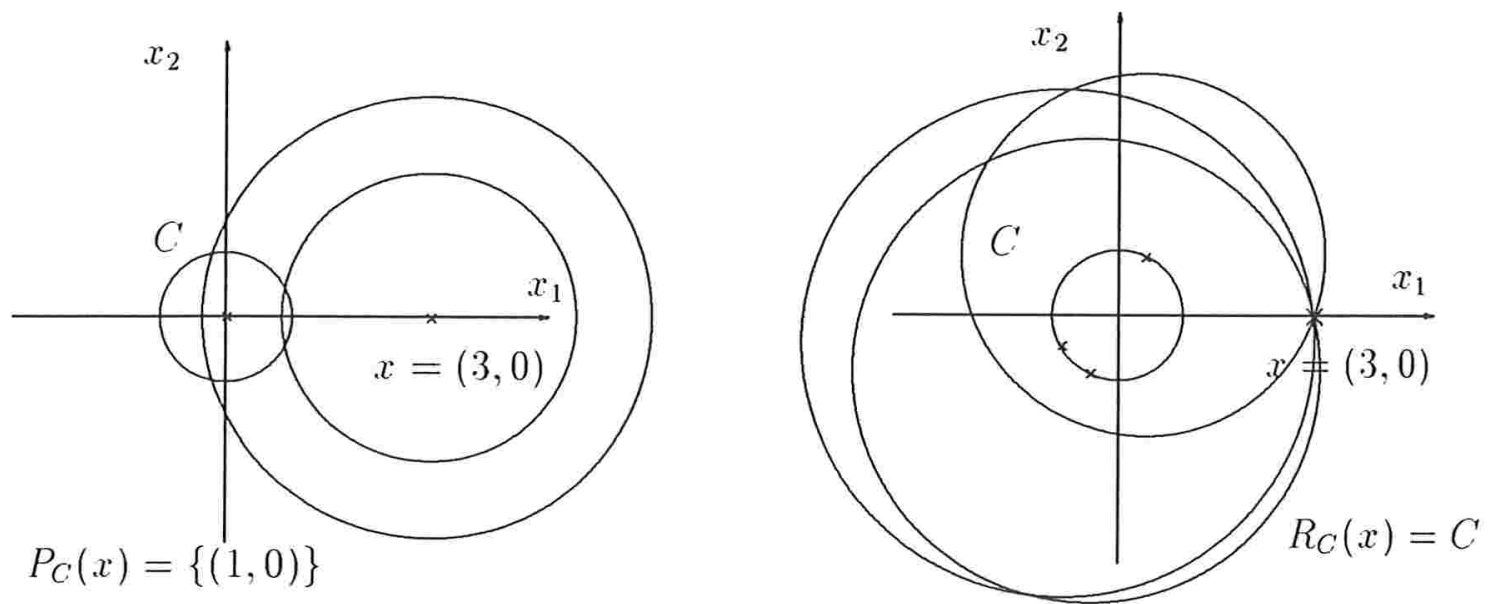

Figura 1.2. 
Exemplo 1.2.3. Tomemos agora ainda $\mathbb{R}^{2}$, mas com a norma \|\|$_{\infty}$. Consideremos $x$ e $C$ como no primeiro exemplo. Neste caso, os conjuntos também não são iguais, pois

$$
P_{C}(x)=[-1,1] \times\{0\} \text { e } R_{C}(x)=\{(0,0)\}
$$
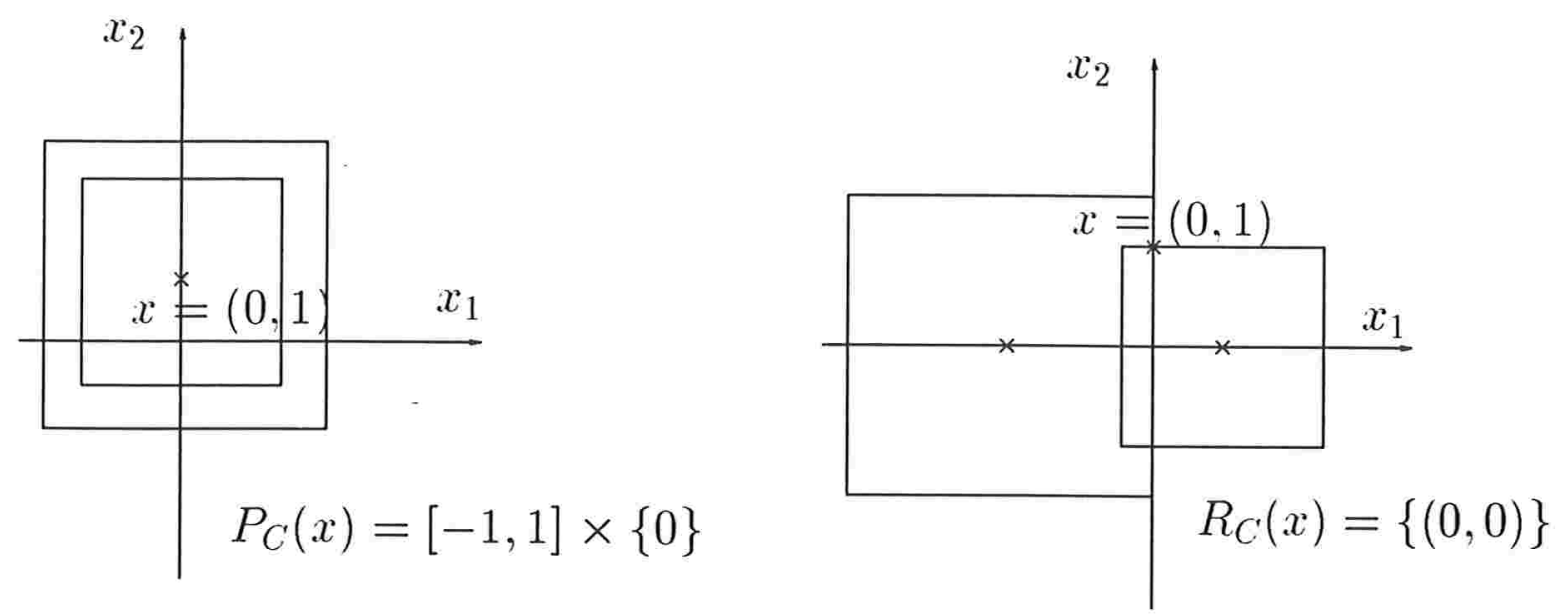

Figura 1.3.

Exemplo 1.2.4. Novamente com $\mathbb{R}^{2}, x=(0,1)$ e $C$ o eixo $x_{1}$, só que neste caso vamos considerar a norma \|\|$_{1}$. O resultado é o oposto ao obtido no caso anterior:

$$
P_{C}(x)=\{(0,0)\} \text { e } R_{C}(x)=[-1,1] \times\{0\} .
$$
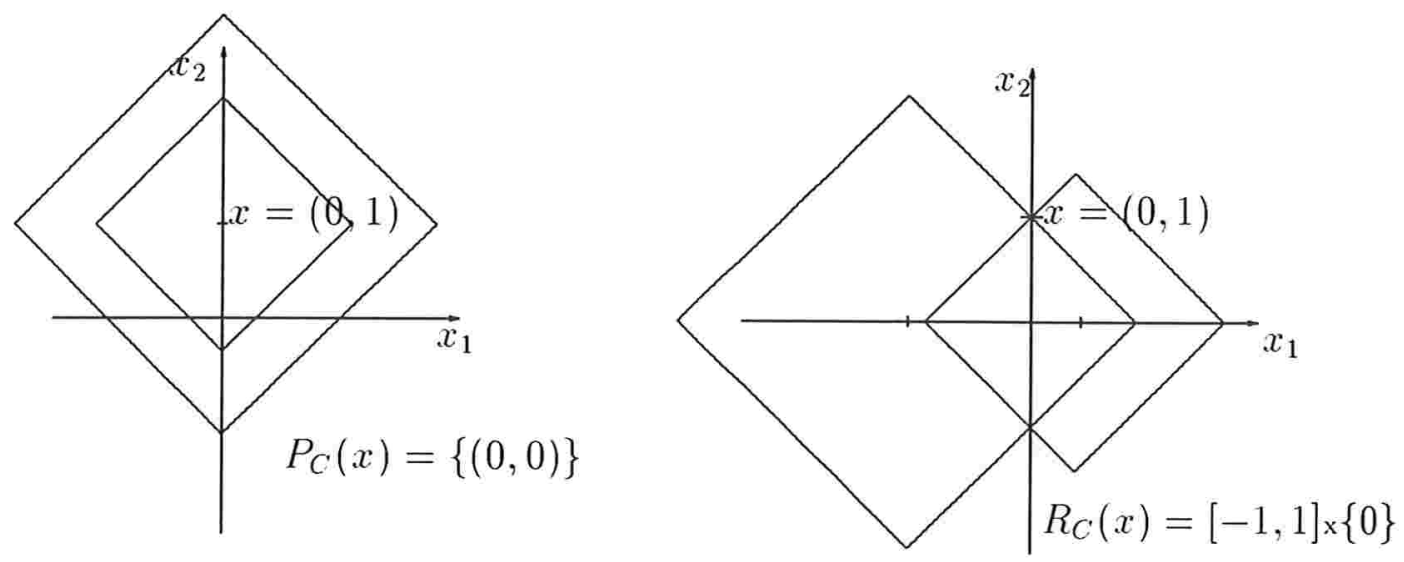

Figura 1.4 . 
Exemplo 1.2.5. Sejam $X=\left(C[0.1],\|\|_{2}\right), C=\mathcal{P}_{1}$ (conjunto dos polinômios de grau no máximo 1) e $y=x^{4}$. Vamos achar $P_{C}(y)$ e $R_{C}(y)$.

Procuraremos $P_{C}(y)$, ou seja, as funções $l \in \mathcal{P}_{1}$ tais que

$$
\|y-l\|_{2} \leq\|y-g\|_{2} \text { para toda } g \in C .
$$

Em outras palavras, devemos achar as funções da forma $a x+b$, tais que

$$
\int_{0}^{1}\left(x^{4}-(a x+b)\right)^{2} d x \text { seja mínima. }
$$

Fazendo operações

$$
\int_{0}^{1}\left(x^{4}-(a x+b)\right)^{2} d x=\frac{1}{9}-\frac{a}{3}-\frac{2 b}{5}+\frac{a^{2}}{3}+a b+b^{2} .
$$

Chamando

$$
M(a, b)=\frac{1}{9}-\frac{a}{3}-\frac{2 b}{5}+\frac{a^{2}}{3}+a b+b^{2},
$$

obtemos uma função de $\mathbb{R}^{2}$ em $\mathbb{R}$, da qual devemos achar o mínimo (se existir) em todo $\mathbb{R}^{2}$.

As derivadas parciais da $M$ são

$$
M_{a}(a, b)=-\frac{1}{3}+\frac{2 a}{3}+b
$$

$\mathrm{e}$

$$
M_{b}(a, b)=-\frac{2}{5}+a+2 b,
$$

donde deduz-se que existe uma única $l_{0}(x) \in P_{C}(y)$, que é

$$
l_{0}(x)=\frac{4}{5} x-\frac{1}{5} .
$$

Agora vamos achar $R_{C}(y)$, ou seja, as funções da forma $a x+b$ tais que

$$
\|a x+b-g\|_{2} \leq\left\|x^{4}-g\right\|_{2} \text { para toda } g \in \mathcal{P}_{1},
$$

o que equivale a resolver

$$
\|a x+b-g\|_{2}^{2} \leq\left\|x^{4}-g\right\|_{2}^{2} \text { para toda } g \in \mathcal{P}_{1} .
$$


Queremos então que para quaisquer $m, n \in \mathbb{R}$

$$
\int_{0}^{1}[(a x+b)-(m x+n)]^{2} d x \leq \int_{0}^{1}\left(x^{4}-m x-n\right)^{2} d x
$$

ou ainda,

$$
\int_{0}^{1}(a x+b)^{2} d x-2 \int_{0}^{1}\left[a m x^{2}+(a n+b m) x\right] d x \leq \int_{0}^{1} x^{8} d x-2 \int_{0}^{1}\left[x^{4}(m x+n)\right] d x
$$

donde,

$$
m\left(-\frac{2 a}{3}-b+\frac{1}{3}\right)+n\left(-a-2 b+\frac{2}{5}\right)+a^{2}+a b+b^{2}-\frac{1}{9} \leq 0 .
$$

Em resumo temos

$$
\left\{\begin{array}{l}
-\frac{2 a}{3}-b+\frac{1}{3}=0 \\
-a-2 b+\frac{2}{5}=0
\end{array}\right.
$$

e além disso $a^{2}+a b+b^{2}-\frac{1}{9} \leq 0$.

Resolvendo o sistema linear acima obtemos que $a=\frac{4}{5}$ e $b=-\frac{1}{5}$ e tais valores satisfazem $\left({ }^{1}\right)$. Logo a melhor coaproximação de $y=x^{4}$ em $\mathcal{P}_{1}$ é

$$
f_{0}(x)=\frac{4}{5} x-\frac{1}{5},
$$

a qual é igual à melhor aproximação (ver equação 1.3 e figura 1.5).

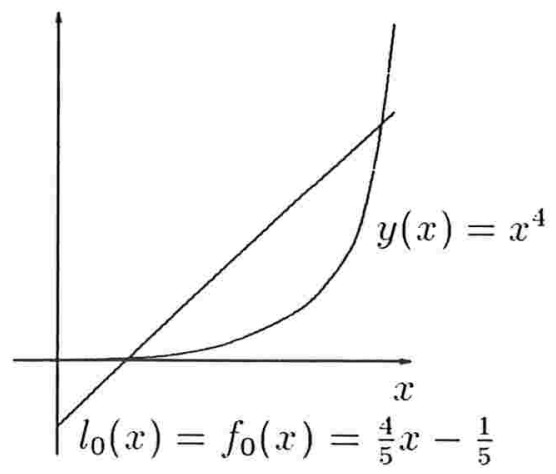

Figura 1.5. 
Exemplo 1.2.6. Consideremos agora $X=\left(C[0,1],\|\|_{\infty}\right)$ e $C$ e $y$ como no exemplo anterior.

Procuraremos $P_{C}(y)$, ou seja, as funções $l$ da forma $l(x)=a x+b$ tais que

$$
\max _{0 \leq x \leq 1}\left|x^{4}-a x-b\right| \text { seja mínimo. }
$$

Os extremos de $h(x)=x^{4}-a x-b$ em $[0,1]$ podem ser

$$
\left\{\begin{array}{cl}
|b| & \text { em } x=0 \\
|a+b-1| & \text { em } x=1 \\
\left|3\left(\frac{a}{4}\right)^{\frac{4}{3}}+b\right| & \text { em } x=\left(\frac{a}{4}\right)^{\frac{1}{3}}, \text { se }\left(\frac{a}{4}\right)^{\frac{1}{3}} \text { pertence a }[0,1] .
\end{array}\right.
$$

Daí, minimizando-os, obtemos como únicos resultados $a=1$ e $b=-\frac{3}{8 \sqrt[3]{4}}$.

Portanto

$$
l_{0}(x)=x-\frac{3}{8 \sqrt[3]{4}}
$$

Agora acharemos $R_{C}(y)$.

Precisamos achar $g_{0} \in \mathcal{P}_{1}$ tal que

$$
\left\|g-g_{0}\right\|_{\infty} \leq\left\|x^{4}-g\right\|_{\infty} \quad \text { para todo } g \in \mathcal{P}_{1},
$$

ou seja, achar $a, b \in \mathbb{R}$ tais que

$$
\|m x+n-(a x+b)\|_{\infty} \leq\left\|x^{4}-m x-n\right\|_{\infty} \quad \text { para quaisquer } \quad m, n \in \mathbb{R},
$$

o que é equivalente a

$$
\max _{x \in[0,1]}|(m-a) x+n-b| \leq \max _{x \in[0,1]}\left|x^{4}-m x-n\right| \text { para quaisquer } m, n \in \mathbb{R} .
$$

Esquematicamente, expressaremos a desigualdade acima da seguinte maneira:

$$
\max (I) \leq \max (I I)
$$

As possibilidades de máximos são:

$$
\left\{\begin{array}{l}
\text { em }(I), \quad \text { para } x=0, \quad \text { ou } x=1 \\
\text { em }(I I), \quad \text { para } x=0, \quad x=\left(\frac{m}{4}\right)^{\frac{1}{3}} \text { ou } x=1 .
\end{array}\right.
$$


Em outras palavras, a desigualdade (1.4) é equivalente a

$$
\max \{|n-b|,|m-a+n-b|\} \leq \max \left\{|n|,\left|-\frac{3 m^{4 / 3}}{4^{4 / 3}}-n\right|,|1-m-n|\right\} .
$$

Vamos resolver o problema separando nos seguintes casos:

(i) $m \leq 0, \quad$ (ii) $m \geq 4 \quad$ e $\quad$ (iii) $0<m<4$.

(i) Se $n \leq \frac{1-m}{2}$, então $|n| \leq|n-(1-m)|$, e portanto

$$
\max (I I)=|1-m-n| .
$$

Analogamente se $n \geq \frac{1-m}{2}$ temos $\max (I I)=|n|$.

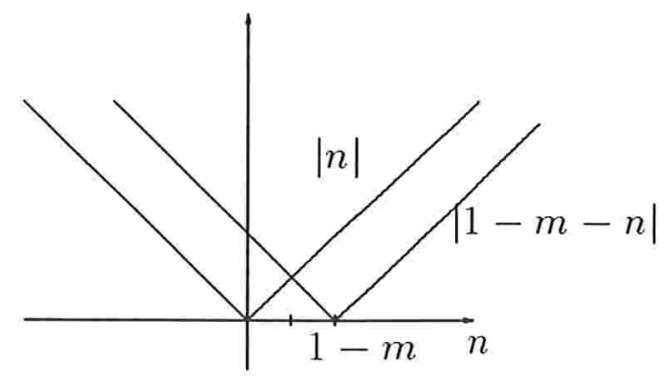

Figura 1.6.

(ii) Neste caso se $n \geq \frac{1-m}{2}$ temos que $|n| \leq|1-m-n|$, e portanto

$$
\max (I I)=|n+m-1| \text {. }
$$

Analogamente se $n<\frac{1-m}{2}$ temos $\max (I I)=|n|$.

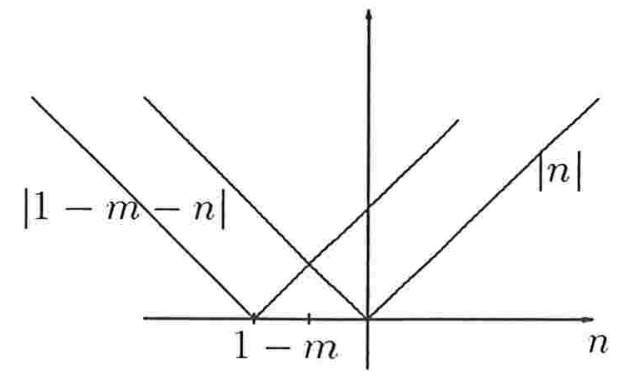

Figura 1.7. 
(iii) Vamos comparar as funçòes $f(m)=1-m$ e $g(m)=-3\left(\frac{m}{4}\right)^{\frac{4}{3}}$. Fazendo o estudo delas é possível concluir que $f(m)>g(m)$ para todo $0<m<4$ (ver figura $1.8)$.

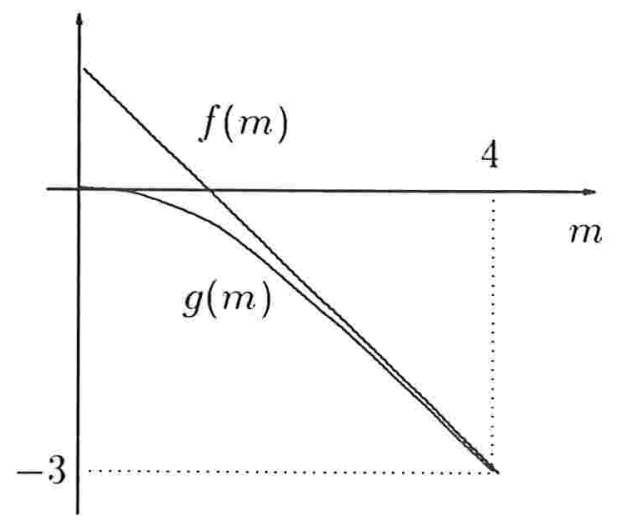

\section{Figura 1.8.}

Como $m-1$ pode ser tanto positivo como negativo, precisamos estudar mais dois novos casos:

(iv) $0<m<1 \quad$ e $\quad$ (v) $1 \leq m<4$.

(iv) Se $n \leq \frac{3\left(\frac{m}{4}\right)^{\frac{4}{3}}+m-1}{2}$, temos

$$
\max (I I)=\left|3\left(\frac{m}{4}\right)^{\frac{4}{3}}-n\right| .
$$

Analogamente se $n>\frac{3\left(\frac{m}{4}\right)^{\frac{4}{3}}+m-1}{2}$, temos

$$
\max (I I)=|1-m-n| .
$$

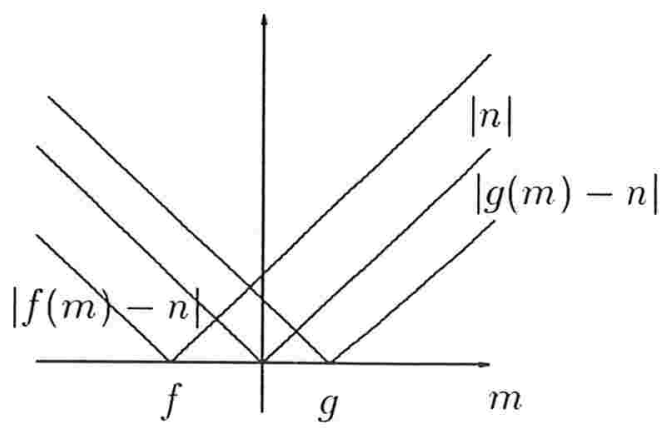

Figura 1.9. 
(v) Se $n \leq \frac{3\left(\frac{m}{4}\right)^{\frac{4}{3}}}{2}$. temos

$$
\max (I I)=|1-m-n|,
$$

e se $n>\frac{3\left(\frac{m}{4}\right)^{\frac{4}{3}}}{2}$, temos

$$
\max (I I)=|n|
$$

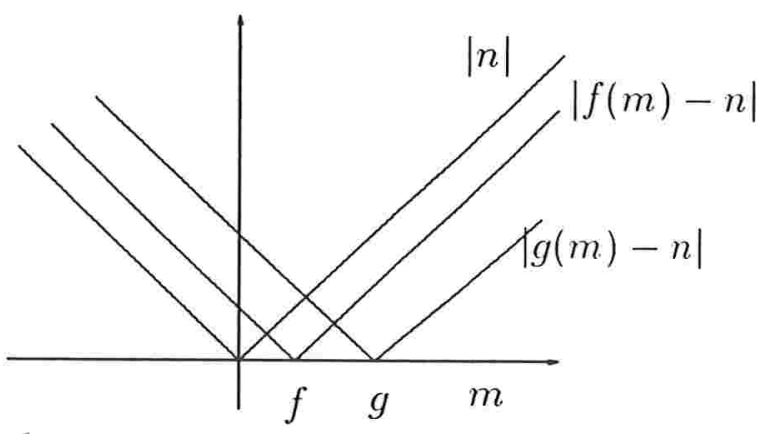

Figura 1.10.

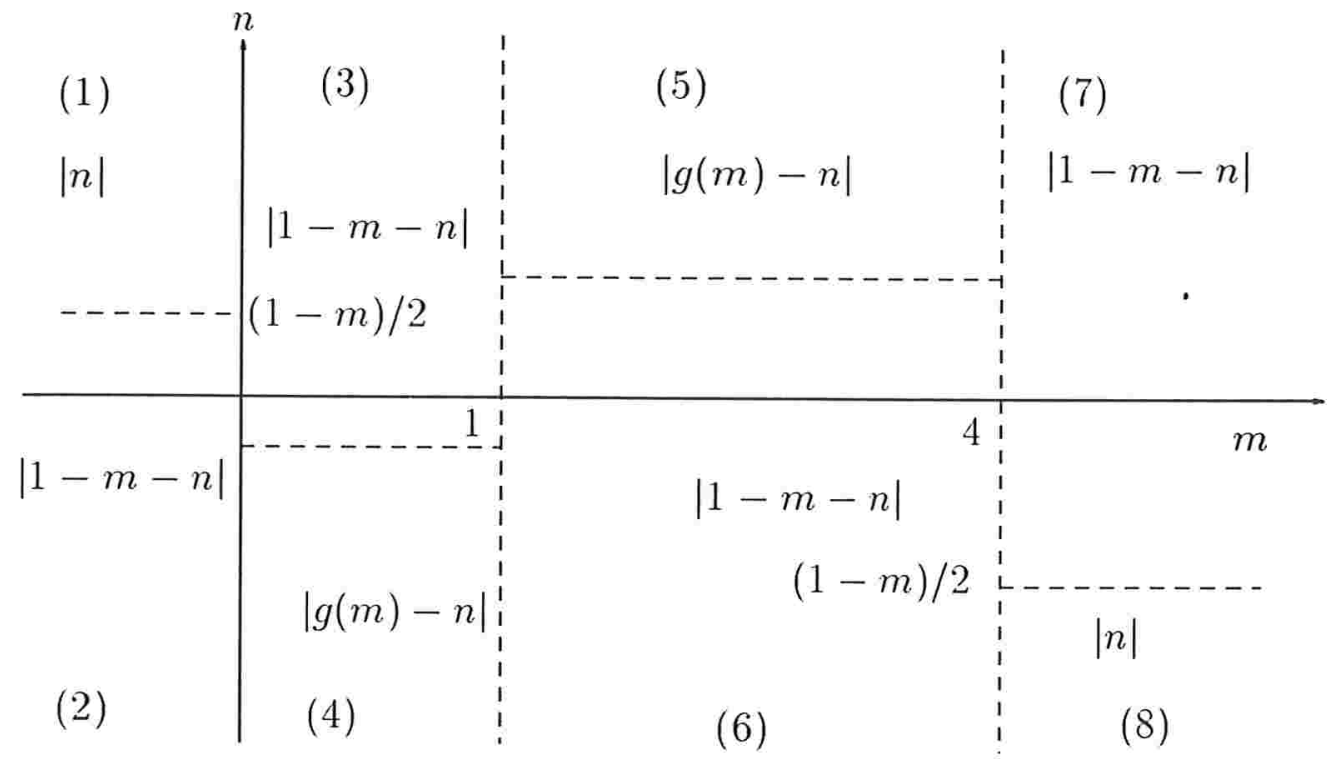

Figura 1.11. Resumo dos resultados do exemplo 1.2.6

Agora estamos aptos a resolver a inequação (1.4), procurando $a$ e $b \in \mathbb{R}$ que a satisfaçam. Usando os resultados de (1),(2), (7) e (\$), concluímos que a única função que satisfaz essas condições é $g_{0}(x)=x$. Vejamos se satisfaz a definição (outro caminho é verificar que satisfaz todas as outras condições). 
A função $g_{0}$ pertence a $R_{C}(y)$ se e somente se

$$
\|(m-1) x+n\|_{\infty} \leq\left\|x^{4}-m x-n\right\|_{\infty} \quad \text { para quaisquer } \quad m, n \in \mathbb{R},
$$

ou seja,

$$
\max \{|n|,|1-m-n|\} \leq \max \{|n|,|1-m-n|,|g(m)-n|\},
$$

o que é óbvio. Portanto $g_{0}(x)=x$ é a melhor coaproximação de $y(x)=x^{4}$ em $\mathcal{P}_{1}$ (ver figura 1.12).

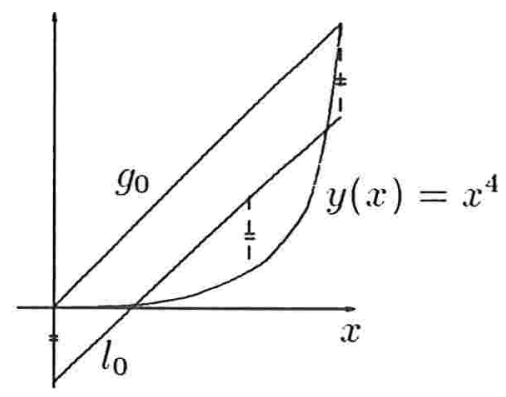

\section{Figura 1.12.}

No capítulo 4 determinaremos uma forma fácil de achar as melhores coaproximações polinômicas de uma função contínua em $[0,1]$ que confirma este resultado.

Exemplo 1.2.7. Seja $X=\left(C[0,1],\|\|_{\infty}\right)$. Queremos achar a melhor aproximação e a melhor coaproximação de $f(x)=e^{x}$ no subespaço $C$ das funçoes constantes (ver uma generalização deste exemplo na proposição 4.2.9).

Procuraremos $P_{C}(f)$.

Devemos achar $a \in \mathbb{R}$ tal que

$$
\max _{0 \leq x \leq 1}\left|e^{x}-a\right| \quad \text { seja mínimo. }
$$

A resposta é muito simples: $a=\frac{\epsilon+1}{2}$ é o único real que satisfaz tal condição. Portanto $f_{0}(x)=\frac{\epsilon+1}{2}$ é a melhor aproximação da função $f(x)=e^{x}$ no subespaço $C$ das funções constantes.

Agora procuraremos $R_{C}(f)$.

Devemos achar $a \in \mathbb{R}$ tal que

$$
\|a-b\|_{\infty} \leq\left\|e^{x}-b\right\|_{\infty} \quad \text { para todo } b \in \mathbb{R},
$$


ou seja,

$$
|a-b| \leq \max _{0 \leq x \leq 1}\left|e^{x}-b\right| \quad \text { para todo } b \in \mathbb{R} .
$$

Estudemos dois casos:

(i) $b \leq \frac{e+1}{2} \quad$ e $\quad$ (ii) $b>\frac{e+1}{2}$.

(i) Nesta situação a desigualdade fica $|a-b| \leq e-b$,

o que equivale a

$$
1 \leq a \leq e
$$

(ii) Agora a desigualdade fica $|a-b| \leq b-1$,

o que equivale a

$$
1 \leq a \leq e
$$

Daí concluimos que $g_{0}(x)=a$ é melhor coaproximação da função $f(x)=e^{x}$ no subespaço das funções constantes, para qualquer $a \in[1, e]=f([0,1])$.

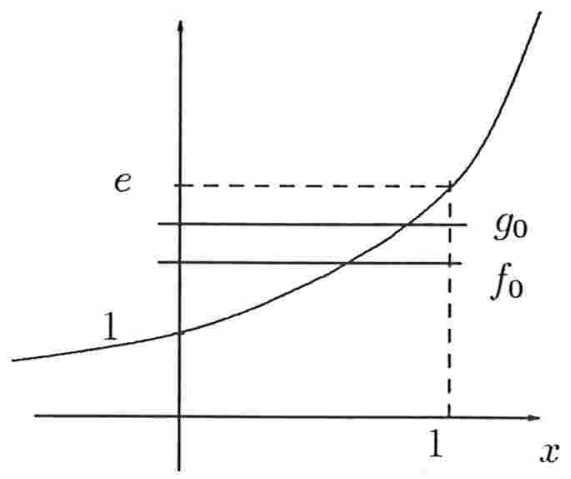

Figura 1.13 .

Observação 1.2.8. Estudando os exemplos anteriores é possível concluir que nem sempre os dois conjuntos com os quais vamos trabalhar coincidem. De fato, eles podem ser bem distintos. É ainda possível deduzir que nos casos onde $P_{C}(x)$ e $R_{C}(x)$ são diferentes também não existe inclusão de um no outro e mesmo que sejam unitários, tais conjuntos, não necessariamente são iguais. No próximo capítulo veremos em que condições tais conjuntos são, com certeza, iguais. 


\section{Capítulo 2}

\section{Existência e Unicidade da Melhor Coaproximação}

O objetivo principal deste capítulo é estudar condições necessárias e suficientes para a existência e unicidade da melhor coaproximação. Além disso veremos exemplos e interpretações geométricas de alguns resultados. Para esses fins, estudaremos algumas propriedades de ortogonalidade e de diferenciabilidade em espaços normados reais.

\subsection{Ortogonalidade.}

Nesta seção vamos estudar o conceito e algumas propriedades de ortogonalidade em espaços normados que vão nos permitir, na seção 2.2, achar condições necessárias e suficientes para a existência da melhor coaproximação. Além disso, os resultados desta seção são muito importantes no desenvolvimento do capítulo 3.

Existem diversos tipos de ortogonalidade em espaços normados, entre os quais a de Birkhoff [Bir35], que será a utilizada neste trabalho. Outros tipos são a isósceles e a pitagórica. Para maiores detalhes ver [Jam45].

Definição 2.1.1. Sejam $X$ um espaço normado real e $x, y \in X$. Dizemos que $x$ é ortogonal a $y$ se para todo $\alpha$ real vale $\|x\| \leq\|x+\alpha y\|$. Denotaremos $x \perp y$. 
Dizemos que o conjunto $Y$ é ortogonal ao conjunto $Z$ se $y \perp z$ para todo $y \in Y$ e para todo $z \in Z$. Denotaremos $Y \perp Z$.

A seguir veremos um exemplo simples que mostra que a ortogonalidade não é necessariamente recíproca e, em seguida, que em espaços pré-hilbertianos é.

Exemplo 2.1.2. Sejam $X=\left(\mathbb{R}^{2},\|\|_{1}\right), x=(1,0)$ e $y=(1,1)$. Vamos provar que $x \perp y$ mas $y \not \perp x$.

$$
\|x+\lambda y\|_{1}=\|(1,0)+(\lambda, \lambda)\|_{1}=|1+\lambda|+|\lambda| .
$$

Então $x \perp y$ pois $|1+\lambda|+|\lambda| \geq 1=\|x\|_{1}$ para todo $\lambda \in \mathbb{R}$.

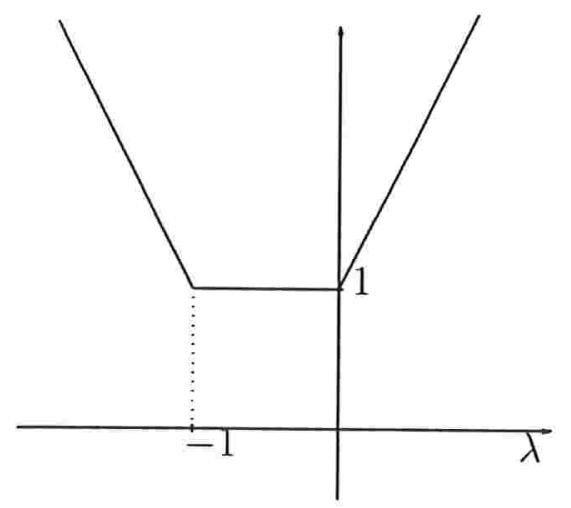

Figura 2.1. $f(\lambda)=|1+\lambda|+|\lambda|$.

Para ver que $y \not \not x$ basta observar que se tomarmos $\lambda=-1$ não é verdade que $\|y\|_{1} \leq\|y+\lambda x\|_{1}$, pois teríamos $2 \leq 1$.

Proposição 2.1.3. Sejam X um espaço pré-hilbertiano, $x$ e y em $X$. Então

$$
x \perp y \Longleftrightarrow<x, y>=0 .
$$

\section{Prova :}

$(\Rightarrow)$

Sabemos por hipótese que $x \perp y$, o que por definição significa que para todo $\lambda \in \mathbb{R}$

$$
\|x\| \leq\|x+\lambda y\| \text {. }
$$


Usando isso temos que para todo $\lambda \in \mathbb{R}$

$$
\|x\|^{2} \leq\|x+\lambda y\|^{2}=<x+\lambda y, x+\lambda y>=\|x\|^{2}+2 \lambda<x, y>+\lambda^{2}\|y\| .
$$

Em resumo temos que para todo $\lambda \in \mathbb{R}$

$$
\lambda^{2}\|y\|+2 \lambda<x, y>\geq 0 .
$$

Logo,

$$
<x, y>=0 \text {. }
$$

$(\Leftarrow)$

Primeiramente, observemos que se $x=0$, a afirmação é óbvia. Suponhamos então que temos $x \neq 0$ e que $\langle x, y\rangle=0$. Portanto para todo $\lambda \in \mathbb{R},\|x\|^{2}=\langle x, x+\lambda y\rangle$. Mas pela desigualdade de Cauchy-Schwarz temos que

$$
\|x\|^{2}=<x, x+\lambda y>\leq\|x\|\|x+\lambda y\| .
$$

Logo,

$$
\|x\| \leq\|x+\lambda y\|
$$

Corolário 2.1.4. Sejam X um espaço pré-hilbertiano, $x$ e y em $X$ tais que $x \perp y$. Então $y \perp x$.

\section{Prova :}

Imediata. Basta observar que como o corpo é $\mathbb{R},\langle x, y\rangle=\langle y, x\rangle$ e utilizar a proposição 2.1.3.

Lema 2.1.5. A ortogonalidade é uma propriedade homogênea, i.e., se $x \perp y$ então $a x \perp$ by para quaisquer $a, b \in \mathbb{R}$.

\section{Prova :}

Sabemos da hipótese que $x \perp y$, ou seja, para todo $\lambda \in \mathbb{R}$ temos que $\|x\| \leq\|x+\lambda y\|$ e queremos provar que $a x \perp$ by para quaisquer $a, b \in \mathbb{R}$. Note-se que se $a=0$ ou $b=0$ nada temos a provar. Por isso trabalharemos com os dois distintos de zero.

Dados $a$ e $b \in \mathbb{R}^{\star}$, para cada $\lambda^{\prime} \in \mathbb{R}$ escolhemos $\lambda=\frac{\lambda^{\prime} b}{a}$ e substituindo na hipótese 
obtemos que

$$
\|x\| \leq\left\|x+\frac{\lambda^{\prime} b}{a} y\right\|
$$

ou seja.

$$
\|x\| \leq\left\|\frac{a x+\lambda^{\prime} b y}{a}\right\|
$$

Portanto

$$
|a|\|x\| \leq\left\|a x+\lambda^{\prime} b y\right\|
$$

e daí

$$
\|a x\| \leq\left\|a x+\lambda^{\prime} b y\right\|
$$

Lema 2.1.6. Se $f$ é um funcional linear contínuo não nulo em um espaço normado $X$ e $x \in X$, então

$$
|f(x)|=\|f\|\|x\| \Longleftrightarrow x \perp \operatorname{ker}(f) .
$$

\section{Prova :}

\section{$(\Rightarrow)$}

Tomando $h \in \operatorname{ker}(f)$, sabemos que

$$
|f(x)|=|f(x+h)| \leq\|f\|\|x+h\|
$$

e como $|f(x)|=\|f\|\|x\|$ e $\|f\| \neq 0$,

$$
\|x\| \leq\|x+h\| .
$$

Pelo lema 2.1.5, $\|x\| \leq\|x+\alpha h\|$ para todo $\alpha \in \mathbb{R}$. Logo, $x \perp h$.

$(\Leftarrow)$

Sejam $x \perp \operatorname{ker}(f)$ e $|f(x)|=p\|x\|$, com $p \geq 0$. É claro que $p \leq\|f\|$, pois se $p>\|f\|$, teríamos $|f(x)|<p\|x\|$. Vamos mostrar que $p=\|f\|$.

Para todo $h \in \operatorname{ker}(f)$, temos

$$
\|x\| \leq\|x+h\|
$$


e portanto

$$
|f(x+h)|=|f(x)|=p\|x\| \leq p\|x+h\| .
$$

Além disso, temos que todo $y \in X$ pode ser expresso na forma $a x+h$, com $a \in \mathbb{R}$ e $h \in \operatorname{ker}(f)$. Se $a=0,|f(y)| \leq p\|y\|$. Suponhamos que $a \neq 0$. Então temos

$$
|f(y)|=|f(a x+h)|=|a|\left|f\left(x+\frac{1}{a} h\right)\right| \leq|a| p\left\|x+\frac{1}{a} h\right\|=p\|y\| .
$$

Em resumo, para todo $y \in X$

$$
|f(y)| \leq p\|y\|
$$

Logo $p \geq\|f\| \mathrm{e}$

$$
p=\|f\|
$$

Observação 2.1.7. Se $f=0$ o resultado não é válido, pois basta tomar $x \neq 0$ e obviamente não é verdade que $x \perp x$.

Proposição 2.1.8. Sejam $x$ e y dois pontos de um espaço normado X'.

Então são satisfeitas as seguintes propriedades:

(i) Existe $a \in \mathbb{R}$ tal que $x \perp a x+y$.

(ii) Para a como em $(i)$ e $x \neq 0,|a| \leq\|y\| /\|x\|$.

(iii) Para $x \neq 0$, o conjunto $M=\{a \in \mathbb{R}: x \perp a x+y\}$ tem máximo $B$ e mínimo A.

Prova :

(i) Se $x=0$, o resultado é trivial. Se $x \neq 0$, pelo Teorema de Hahn-Banach sabemos que existe $f \in X^{*}$ não nulo tal que $|f(x)|=\|f\|\|x\|$. Pela proposição anterior só precisamos mostrar que $a x+y \in k \in r(f)$, para algum $a \in \mathbb{R}$.

Vejamos:

$$
f(a x+y)=0 \Longleftrightarrow a f(x)+f(y)=0 .
$$

Como $f$ e $x$ não são nulos e $|f(x)|=\|f\|\|x\|$, temos que $f(x) \neq 0$, e tomando $a=-\frac{f(y)}{f(x)}$ a parte (i) está provada. 
(ii) Sejam $x \neq 0$ e $a$ tais que $x \perp a x+y$. Se $a=0$, a afirmação é óbvia. Portanto vamos supor que $a \neq 0$. Pela definição de ortogonalidade temos

$$
\|x\| \leq\|x+k(a x+y)\| \text { para todo } k \in \mathbb{R} .
$$

Dai, tomando $k=-1 / a$, temos

$$
|a| \leq\|y\| /\|x\|
$$

(iii) Pelos ítems anteriores sabemos que $M=\{a \in \mathbb{R}: x \perp a x+y\}$ é não vazio e limitado. Portanto, $M$ tem sup e inf. Sejam $A=\inf (M)$ e $B=\sup (M)$. Mostraremos que $A$ e $B$ são elementos de $M$. Como $B$ é o $\sup (M)$, podemos escolher $\left\{a_{n}\right\}_{n \in N}$ em $M$, tal que $a_{n} \rightarrow B$. Então, para todo $n \in \mathbb{N}$

$$
\|x\| \leq\left\|x+k\left(a_{n} x+y\right)\right\| \text { para todo } k \in \mathbb{R}
$$

Logo, com $n \rightarrow+\infty$, conclui-se que $B \in M$.

Analogamente temos $A \in M$.

Lema 2.1.9. Sejam $x$ e y em um espaço normado $X$, e $A$ e $B$ em $\mathbb{R}$ tais que $A \leq B$, $x \perp A x+y$ e $x \perp B x+y$. Então $x \perp a x+y$ para todo $a \in[A, B]$.

\section{Prova :}

Sejam $a \in[A, B]$ e $k \in \mathbb{R}$. Queremos mostrar que

$$
\|x+k(a x+y)\| \geq\|x\| .
$$

Se $a=A$ ou $a=B$ ou $A=B$, não há nada a provar. Suponhamos então que $a \in] A, B[$.

Para $k \geq 0$

$$
\begin{gathered}
\|x+k(a x+y)\|=\|x+k(a x+y)+k A x-k A x\|=\|x(1+k(a-A))+k(A x+y)\| \geq \\
\geq\|x(1+k(a-A))\| \geq\|x\|, \quad \text { pelo lema } 2.1 .5 \text { e porque } 1+k(a-A) \geq 1 .
\end{gathered}
$$


Para $k<0$

$\|x+k(a x+y)\|=\|x+k(a x+y)+k B x-k B x\|=\|x(1+k(a-B))+k(B x+y)\| \geq$

$\geq\|x(1+k(a-B))\| \geq\|x\|$, novamente pelo lema 2.1 .5 e porque $1+k(a-B) \geq 1$.

Portanto

$$
x \perp a x+y \quad \text { para todo } a \in] A, B[\text {. }
$$

Lema 2.1.10. Sejam $X$ um espaço normado, $x$ e $y \in X$ e $a \in \mathbb{R}$.

Então

$$
\lim _{\lambda \rightarrow+\infty}(\|(\lambda+a) x+y\|-\|\lambda x+y\|)=a\|x\| .
$$

Prova :

Usando que $\lambda+a>0$ para $\lambda$ suficientemente grande e positivo e que $\frac{\lambda}{\lambda+a}+\frac{a}{\lambda+a}=1$, temos

$$
\begin{aligned}
\|(\lambda+a) x+y\| & =\frac{\lambda}{\lambda+a}\|(\lambda+a) x+y\|+\frac{a}{\lambda+a}\|(\lambda+a) x+y\|= \\
& =\left\|\lambda x+\frac{\lambda}{\lambda+a} y\right\|+a\left\|x+\frac{y}{\lambda+a}\right\| .
\end{aligned}
$$

Então

$$
\|(\lambda+a) x+y\|-\|\lambda x+y\|=\left\|\lambda x+\frac{\lambda}{\lambda+a} y\right\|-\|\lambda x+y\|+a\left\|x+\frac{y}{\lambda+a}\right\|,
$$

mas

$$
\left|\left\|\lambda x+\frac{\lambda}{\lambda+a} y\right\|-\|\lambda x+y\|\right| \leq\left\|\left(\frac{\lambda}{\lambda+a}-1\right) y\right\|=\left\|\frac{a}{\lambda+a} y\right\| .
$$

Portanto, como $\left\|\frac{a}{\lambda+a} y\right\| \rightarrow 0$ se $\lambda \rightarrow+\infty$, temos

$$
\left|\left\|\lambda x+\frac{\lambda}{\lambda+a} y\right\|-\|\lambda x+y\|\right| \rightarrow 0 \text { se } \lambda \rightarrow+\infty .
$$


Além disso, como

$$
a\left\|x+\frac{y}{\lambda+a}\right\| \rightarrow a\|x\| \text { se } \lambda \rightarrow+\infty
$$

concluimos que

$$
\lim _{\lambda \rightarrow+\infty}(\|(\lambda+a) x+y\|-\|\lambda x+y\|)=a\|x\| .
$$

Lema 2.1.11. Sejam $x$ e y em um espaço normado $X$ tais que $\|x+y\|=\|x-y\|$, e seja $k \in \mathbb{R}$ tal que $|k| \geq 1$. Então

(i) $\|x+y\| \leq\|x+k y\| \in\|x-y\| \leq\|x+k y\|$;

(ii) $\|x+k y\| \geq\|x\|$.

\section{Prova :}

(i) Basta provar a primeira afirmação, pois $\|x-y\|=\|x+y\|$.

Como $x+k y=\frac{1}{2}(k+1)(x+y)-\frac{1}{2}(k-1)(x-y)$, pela desigualdade triangular, temos

$$
\left\|\frac{1}{2}(k+1)(x+y)\right\| \leq\|x+k y\|+\left\|\frac{1}{2}(k-1)(x-y)\right\|
$$

e

$$
\left\|\frac{1}{2}(k-1)(x-y)\right\| \leq\|x+k y\|+\left\|\frac{1}{2}(k+1)(x+y)\right\| .
$$

Se $k \geq 1$, por (2.1) temos

$$
\begin{gathered}
\|x+k y\| \geq\left\|\frac{1}{2}(k+1)(x+y)\right\|-\left\|\frac{1}{2}(k-1)(x-y)\right\|= \\
=\frac{1}{2}(k+1)\|(x+y)\|-\frac{1}{2}(k-1)\|(x-y)\| .
\end{gathered}
$$

Como por hipótese $\|x+y\|=\|x-y\|$, concluimos que $\|x+y\| \leq\|x+k y\|$ para todo $k \geq 1$. 
Se $k \leq-1$, por $(2.2)$ temos

$$
\begin{gathered}
\|x+k y\| \geq\left\|\frac{1}{2}(k-1)(x-y)\right\|-\left\|\frac{1}{2}(k+1)(x+y)\right\|= \\
=-\frac{1}{2}(k-1)\|(x-y)\|+\frac{1}{2}(k+1)\|(x+y)\| .
\end{gathered}
$$

Novamente usando a hipótese, deduz-se $\|x+y\| \leq\|x+k y\|$ para todo $k \leq-1$. Logo,

$$
\|x+y\| \leq\|x+k y\| \text { para todo }|k| \geq 1 .
$$

(ii) Como $2 x=(x+y)+(x-y)$, utilizando a desigualdade triangular e a parte (i), temos

$$
2\|x\| \leq 2\|x+k y\|
$$

Proposição 2.1.12. Sejam $x \neq 0$ e y dois vetores de um espaço normado $X$. Então

(i) $\lim _{\lambda \rightarrow+\infty}(\|\lambda x+y\|-\|\lambda x\|)=-A\|x\|$;

(ii) $\lim _{\lambda \rightarrow+\infty}(\|\lambda x\|-\|\lambda x-y\|)=-B\|x\|$,

onde A e B são como na proposição 2.1.8.

\section{Prova :}

(i) Vamos mostrar que para toda seqüência $\left\{\lambda_{i}\right\}_{i \in N}$, com $\lambda_{i} \rightarrow+\infty$ quando $i \rightarrow+\infty$, se existe

$$
\lim _{i \rightarrow+\infty}\left(\left\|\lambda_{i} x+y\right\|-\left\|\lambda_{i} x\right\|\right)
$$

ele é igual a $-A\|x\|$. Como $|\|\lambda x+y\|-\|\lambda x\||$ está limitado por $\|y\|$, podemos tomar duas seqüências $\left\{\alpha_{i}\right\}$ e $\left\{\beta_{i}\right\}$ tais que $\alpha_{i} \rightarrow+\infty, \beta_{i} \rightarrow+\infty$ quando $i \rightarrow+\infty$ e

$$
\lim _{i \rightarrow+\infty}\left(\left\|\alpha_{i} x+y\right\|-\left\|\alpha_{i} x\right\|\right)=\limsup (\|\lambda x+y\|-\|\lambda x\|)
$$


$$
\lim _{i \rightarrow+\infty}\left(\left\|\beta_{i} x+y\right\|-\left\|\beta_{i} x\right\|\right)=\liminf (\|\lambda x+y\|-\|\lambda x\|) .
$$

Assim obtemos

$$
\lim _{\lambda \rightarrow+\infty}(\|\lambda x+y\|-\|\lambda x\|)=-A\|x\| .
$$

Seja então $\left\{\lambda_{i}\right\}_{i}$ tal que $\lim _{i \rightarrow+\infty} \lambda_{i}=+\infty$ e $\lim _{i \rightarrow+\infty}\left(\left\|\lambda_{i} x+y\right\|-\left\|\lambda_{i} x\right\|\right) \in \mathbb{R}$. Digamos que $\lim _{i \rightarrow+\infty}\left(\left\|\lambda_{i} x+y\right\|-\left\|\lambda_{i} x\right\|\right)=-r\|x\|$ para algum $r \in \mathbb{R}$. Vamos mostrar que $r=A$.

Somando e subtraindo $\left\|\lambda_{i} x+(A x+y)\right\|+A\|x\|$ na expressão do limite a igualdade fica

$$
\begin{aligned}
\lim _{i \rightarrow+\infty}-\left\|\lambda_{i} x+(A x+y)\right\|+\left\|\lambda_{i} x+y\right\| & +A\|x\|+\left\|\lambda_{i} x+(A x+y)\right\|-\left\|\lambda_{i} x\right\|-A\|x\|= \\
& =-r\|x\|,
\end{aligned}
$$

que pelo lema 2.1.10 fica

$$
\lim _{i \rightarrow+\infty}\left(\left\|\lambda_{i} x+(A x+y)\right\|-\left\|\lambda_{i} x\right\|-A\|x\|\right)=-r\|x\|
$$

Como $A$ é tal que $x \perp A x+y$, temos $\left\|\lambda_{i} x+(A x+y)\right\| \geq\left\|\lambda_{i} x\right\|$, e então

$$
-A\|x\| \leq-r\|x\| \text {. }
$$

Logo,

$$
r \leq A \text {. }
$$

Voltando a

$$
\lim _{i \rightarrow+\infty}\left(\left\|\lambda_{i} x+y\right\|-\left\|\lambda_{i} x\right\|\right)=-r\|x\|,
$$

para todo $\varepsilon>0$ temos $N$ tal que se $i>N$ vale

$$
\left|\left\|\lambda_{i} x+y\right\|-\left\|\lambda_{i} x\right\|+r\|x\|\right|<\varepsilon\|x\|,
$$

ou ainda, para $i>N$,

$$
\left\|\lambda_{i} x+y\right\|-\left\|\lambda_{i} x\right\|+r\|x\|=\epsilon_{i}\|x\| \quad \text { com } \quad\left|e_{i}\right|<\varepsilon .
$$


Dai, também temos

$$
\left\|\lambda_{i} x+y\right\|=\left\|\lambda_{i} x\right\|-r\|x\|+e_{i}\|x\|=\left\|\left(\lambda_{i}-r+e_{i}\right) x\right\| .
$$

Multiplicando por 2 e reescrevendo a igualdade de forma conveniente, obtemos

$$
\left\|\left[\left(2 \lambda_{i}-r+e_{i}\right) x+y\right]+\left[\left(r-e_{i}\right) x+y\right]\right\|=\left\|\left[\left(2 \lambda_{i}-r+e_{i}\right) x+y\right]-\left[\left(r-e_{i}\right) x+y\right]\right\|,
$$

e, pelo lema 2.1.11, temos para todo $k \in \mathbb{R}$ tal que $|k| \geq 1$,

$$
\left\|\left(2 \lambda_{i}-r+e_{i}\right) x+y+k\left[\left(r-e_{i}\right) x+y\right]\right\| \geq\left\|\left(2 \lambda_{i}-r+e_{i}\right) x+y\right\| .
$$

Seja $p \in \mathbb{R}$ qualquer, escolhemos $N_{1} \geq N$ de modo que $k=\frac{2 \lambda_{i}}{|p|} \geq 1$. Logo, $k$ satisfaz (2.4).

Agora, dividindo por $k$, obtemos

$$
\left\|\frac{\left(2 \lambda_{i}-r+e_{i}\right) x+y}{2 \lambda_{i} / p}+\left(r-e_{i}\right) x+y\right\| \geq\left\|\frac{\left(2 \lambda_{i}-r+e_{i}\right) x+y}{2 \lambda_{i} / p}\right\|,
$$

equivalente a

$$
\left\|p\left(1-\frac{r-e_{i}}{2 \lambda_{i}}\right) x+\frac{p y}{2 \lambda_{i}}+\left(r-e_{i}\right) x+y\right\| \geq\left\|p\left(1-\frac{r-e_{i}}{2 \lambda_{i}}\right) x+\frac{p y}{2 \lambda_{i}}\right\| .
$$

Tomando o limite quando $i \rightarrow+\infty$ e $\varepsilon \rightarrow 0$, obtemos que para qualquer $p \in \mathbb{R}$

$$
\|p x+r x+y\| \geq\|p x\| .
$$

Dividindo por $|p|$ e utilizando o lema 2.1 .5 e a definição temos que $x \perp r x+y$, donde concluimos que

$$
r \geq A
$$

Por (2.3) temos que

$$
r=A \text {. }
$$

(ii) Seja $B$ o maior $b$ satisfazendo que $x \perp b x+y$. Então $-B$ é o menor dos $b$ tais que $x \perp b(-x)-y$, e daí, por (i), temos

$$
\lim _{\lambda \rightarrow+\infty}(\|\lambda x-y\|-\|\lambda x\|)=-(-B)\|x\|
$$

e portanto

$$
\lim _{x \rightarrow+\infty}(\|\lambda x\|-\|\lambda x-y\|)=-B\|x\|
$$


Exemplo 2.1.13. Sejam $X=\left(\mathbb{R}^{2},\|\|_{1}\right), x=(1.0)$ e $y=(0,1)$. Calculemos $A$ e $B$.

$$
\lim _{\lambda \rightarrow+\infty}(\|\lambda x+y\|-\|\lambda x\|)=\lim _{\lambda \rightarrow+\infty}(\|(\lambda, 1)\|-\|(\lambda, 0)\|)=1 .
$$

Portanto, $A=-1$.

Em forma análoga obtemos que $B=1$ e daí

$$
(1,0) \perp(a, 1) \text { para todo } a \in[-1,1] \text {. }
$$

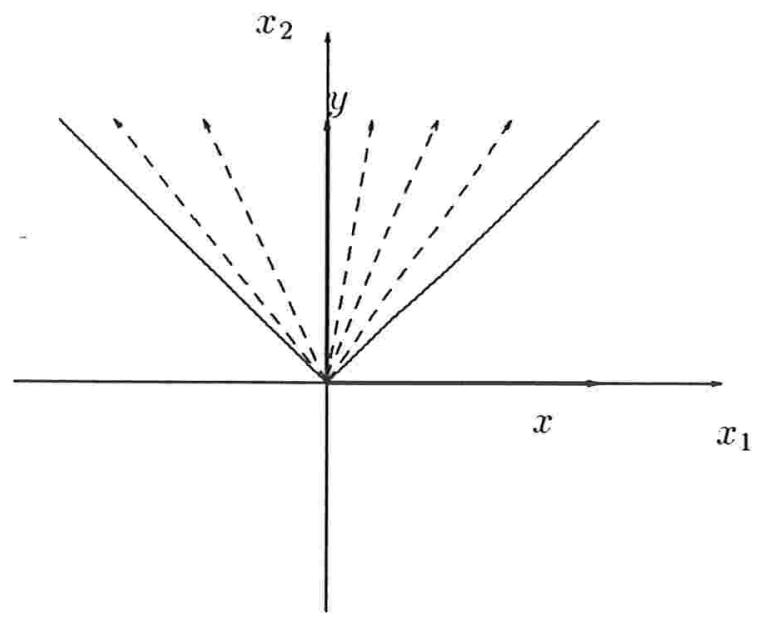

Figura 2.2. 


\subsection{Existência da Melhor Coaproximação.}

Nesta seção, utilizando os resultados de seção 2.1, acharemos condições necessárias e suficientes para a existência da melhor coaproximação. Além disso, demonstraremos uma caracterização dos espaços de Hilbert utilizando a melhor coaproximação (teorema 2.2.13).

Proposição 2.2.1. Sejam $X$ um espaço normado, $G$ um subespaço de $X, g_{0} \mathrm{em} G$ e $x$ em $X$. Então

$$
g_{0} \in P_{G}(x) \Longleftrightarrow x-g_{0} \perp G^{\prime} .
$$

\section{Prova :}

$(\Rightarrow)$

Sabemos que para todo $g \in G$

$$
\left\|x-g_{0}\right\| \leq\|x-g\|
$$

e é preciso provar que para todo $\alpha \in \mathbb{R}$ e para todo $g_{1} \in G$

$$
\left\|x-g_{0}\right\| \leq\left\|x-g_{0}+\alpha g_{1}\right\|
$$

Então, para quaisquer $\alpha \in \mathbb{R}$ e $g_{1} \in G$. consideremos $g=g_{0}-\alpha g_{1}$, que pertence a $G$, que é subespaço. Substituindo em (2.5), obtemos o resultado desejado.

$(\Leftarrow)$

Agora temos

$$
x-g_{0} \perp g_{1} \text { para todo } g_{1} \in G,
$$

o que significa que para todo $\alpha \in \mathbb{R}$ e para todo $g_{1} \in G$ temos

$$
\left\|x-g_{0}\right\| \leq\left\|x-g_{0}+\alpha g_{1}\right\| .
$$

Dado $g \in G$, tomando $\alpha=1$ e $g_{1}=g-g_{0} \in G$ temos que $\left\|x-g_{0}\right\| \leq\|x-g\|$. Logo, $g_{0} \in P_{G}(x)$.

Proposição 2.2.2. Sejam $X$ um espaço normado, $G$ subespaço de $X, g_{0}$ em $G$ e $x$ um ponto de X. Então

$$
g_{0} \in R_{G}(x) \Longleftrightarrow G \perp x-g_{0}
$$




\section{Prova :}

\section{$(\Rightarrow)$}

Temos que provar que para todo $g \in G$, tem-se $g \perp x-g_{0}$, ou seja, que para todo $g \in G$ e para todo $\alpha \in \mathbb{R}$

$$
\|g\| \leq\left\|g+\alpha\left(x-g_{0}\right)\right\| .
$$

Se $\alpha=0$, a desigualdade é óbvia. Podemos então supor que $\alpha \neq 0$.

Como $g_{0} \in R_{G}(x)$, então para todo $g_{1} \in G$

$$
\left\|g_{0}-g_{1}\right\| \leq\left\|x-g_{1}\right\| \text {. }
$$

Para carla $g \in G$, tomamos $g_{1}=g_{0}-\frac{1}{\alpha} g$, que pertence a $G$ porque $G$ é subespaço. Daí temos que para todo $g \in G$

$$
\left\|g_{0}-g_{0}+\frac{1}{\alpha} g\right\| \leq\left\|x-\left(g_{0}-\frac{1}{\alpha} g\right)\right\|,
$$

ou

$$
\frac{1}{|\alpha|}\|g\| \leq\left\|x-g_{0}+\frac{1}{\alpha} g\right\|
$$

Portanto, para todo $g \in G$

$$
\|g\| \leq\left\|g+\alpha\left(x-g_{0}\right)\right\|
$$

donde concluimos que

$$
g \perp x-g_{0} \text { para todo } g \in G \text {. }
$$

$(\Leftarrow)$

Para qualquer $g \in G$ sabemos por hipótese que $g \perp x-g_{0}$, ou seja, para todo $g \in G$ e para todo $\alpha \in \mathbb{R}$ temos

$$
\|g\| \leq\left\|g+\alpha\left(x-g_{0}\right)\right\| .
$$

Para cada $g_{1} \in G$ tomamos $\alpha=1$ e $g=g_{0}-g_{1}$, obtendo $\left\|g_{0}-g_{1}\right\| \leq\left\|x-g_{1}\right\|$ e portanto $g_{0} \in \mathbb{R}_{G}(x)$.

Proposição 2.2.3. Seja X um espaço pré-hilbertiano, Gr um subespaço de $X$ e $x$ um ponto de $X$. Então

$$
P_{G}(x)=R_{G}(x) .
$$




\section{Prova :}

Imediata. Basta observar que pelo corolário 2.1.4 a ortogonalidade é recíproca em espaços pré-hilbertianos e aplicar as proposições 2.2 .1 e 2.2.2.

A relação $g_{0} \in R_{C}(x)$ pode ser vista como um multioperador de $X$ em $\mathcal{P}(C)$, onde os elementos de $X$ que têm imagem não vazia têm um papel muito relevante em nosso estudo. Isso motiva a seguinte difinição.

Definição 2.2.4. Sejam $X$ um espaço normado e $C$ um subconjunto de $X$. Definimos o multioperador $R_{C}: \mathrm{X} \rightarrow \mathcal{F}(C)$ por

$$
R_{C}(x)=\left\{g_{0}: g_{0} \text { é melhor coaproximação de } x \text { em } C\right\},
$$

e $D\left(R_{C}\right)$, como o conjunto dos elementos de $X$ que têm melhor coaproximação em $C$, ou seja,

$$
D\left(R_{C}\right)=\left\{x \in X: R_{C}(x) \neq \emptyset\right\} .
$$

$D\left(R_{G}\right)$ é o domínio "razoável" de $R_{C}$ e por isso é chamado de domínio de $R_{C}$.

Teorema 2.2.5. Sejam X um espaço normado e $G$ um subespaço de $X$ de dimensão 1. Então $D\left(R_{G}\right)=X$.

\section{Demonstração :}

Seja $x \in X$. Se $x$ é um elemento de $G$ nada temos a provar. Suponhamos que $x$ não pertence ao subespaço $G$. Sendo $y \in G$ tal que $[y]=G$, pela proposição 2.1.8 temos que existe $b \in \mathbb{R}$ tal que $y \perp b y+x$.

Tomemos $g_{0}=-b y$, ou seja, $y \perp x-g_{0}$. Pelo lema 2.1.5 temos que $\lambda y \perp x-g_{0}$ para todo $\lambda \in \mathbb{R}$, que por definição significa $G \perp x-g_{0}$. Logo, pela proposição 2.2.2, temos que $g_{0} \in R_{G}(x)$.

Definição 2.2.6. Sejam $X^{\prime}$ um espaço normado, $x \in X$ e $G$ um subespaço de $X$. Definimos $[\{x\} \cup G]$ como o subespaço gerado por $x$ e pelos elementos de $G$. 
Lema 2.2.7. Sejam X espaço normado. Gr um subespaço de $X$ e $x \in X \backslash G$ que tem melhor coaproximação em $G$. Então todo elemento de $[\{x\} \cup G]$ tem melhor coaproximação em $G$.

\section{Prova :}

Sabemos que $x$ tem melhor coaproximação em $G$, i.e., existe $x_{0} \in G$ tal que

$$
\left\|g-x_{0}\right\| \leq\|g-x\| \text { para todo } g \in G .
$$

Tomemos $y \in[\{x\} \cup G]$ qualquer, ou seja, $y=a x+z \operatorname{com} a \in \mathbb{R}$ e $z \in G$. Devemos provar que existe $y_{0}$ tal que

$$
\left\|g-y_{0}\right\| \leq\|g-y\| \text { para todo } g \in G .
$$

Observando que se $a=0$ o lema está satisfeito com $y_{0}=z$, vamos supor que $a \neq 0$. Sendo $y_{0}=a x_{0}+z$, temos que $y_{0} \in G$ e para $g \in G$ qualquer, como $g-z \in G$,

$$
\begin{gathered}
\left\|g-y_{0}\right\|=\left\|g-a x_{0}-z\right\|=|a|\left\|\frac{1}{a}(g-z)-x_{0}\right\| \stackrel{(2.6)}{\leq} \\
\quad \leq|a|\left\|\frac{1}{a}(g-z)-x\right\|=\|g-z-a x\|=\|g-y\| .
\end{gathered}
$$

Então $y_{0}$ é melhor coaproximação de $y \mathrm{em} G$.

Lema 2.2.8. Sejam $G$ e $H$ subespaços de um espaço normado $X$ tais que $G \subset H$. Se $x \in X \backslash H$ tem melhor coaproximação em $H$ e todo elemento de $H$ tem melhor coaproximação em $G$, então $x$ tem melhor coaproximação em $G$. Mais precisamente, se $h_{0} \in R_{H}(x)$ e $g_{0} \in R_{G}\left(h_{0}\right)$, então $g_{0} \in R_{G}(x)$.

\section{Prova :}

Como $x$ tem melhor coaproximação em $H$, sabemos que existe $h_{0} \in H$ tal que

$$
\left\|h-h_{0}\right\| \leq\|h-x\| \quad \text { para todo } h \in H .
$$

Além disso, como todo elemento de $H$ tem melhor coaproximação em $G$, em particular $h_{0}$ a tem, e portanto existe $g_{0} \in G$ tal que

$$
\left\|g-g_{0}\right\| \leq\left\|g-h_{0}\right\| \quad \text { para todo } g \in G .
$$

Como $G \subset H$, a desigualdade (2.7) vale para todo $g \in G$, e daí,

$$
\left\|g-g_{0}\right\| \leq\left\|g-h_{0}\right\| \leq\|g-x\| \text { para todo } g \in G .
$$

Logo, $g_{0} \in G$ é ponto de melhor coaproximação de $x$ em $G$. 
Teorema 2.2.9. Se para todo subespaço próprio $F$ de um espaço normado $X$, existe $x \in X \backslash F$ que tem melhor coaproximação em $F$, então para qualquer subespaço $G$ de $X$ temos que $D\left(R_{G}\right)=X$.

\section{Demonstração :}

Seja $G$ um subespaço de $X$. Podemos supor $G \neq X$, pois $D\left(R_{X}\right)=X$. Consideremos então a família $\sum$ de subespaços de $X$ formados por elementos que contêm $G$ e têm melhor coaproximação em $G$, isto é,

$$
\sum=\left\{U \subset X: U \text { subespaço de } X, G \subset U \subset D\left(R_{G}\right)\right\}
$$

Observemos que $\sum \neq \emptyset$, pois $G \in \sum$. Consideremos em $\sum$ a relação de ordem parcial " $\subset$ " clada pela inclusão entre conjuntos. Como toda cadeia $\left\{U_{\alpha}\right\}$ de $\sum$ está limitada superiormente por $\bigcup_{\alpha} U_{\alpha}$, que é subespaço de $X$ e pertence a $\sum$, temos pelo Lema de Zorn que $\sum$ tem elemento maximal $H$.

Se $H=X$ temos $D\left(R_{G}\right)=X$. Suponhamos que $H \neq X$. Portanto existe $x \in X \backslash H$. que tem melhor coaproximação em $H$. Pelo lema 2.2.7 todo elemento de $[\{x\} \cup H]$ tem melhor coaproximação em $H$ e pelo Lema 2.2.8, como $G \subset H \subset D\left(R_{G}\right)$, temos que todo elemento de $[\{x\} \cup H]$ tem melhor coaproximação em $G$, o que é absurdo pois $H$ é maximal.

Definição 2.2.10. Seja $A \neq \emptyset$ um subconjunto de um espaço normado $X$. $A$ é chamado admissível se pode ser expresso como intersecção de bolas fechadas.

Chamaremos de família completa de centros de um conjunto admissivel $A$ a todo conjunto $S$ tal que

$$
A=\bigcap_{x \in S} \bar{B}_{r(x)}(x), \text { onde } r: S \rightarrow[0,+\infty[.
$$

Um subespaço fechado $D$ é chamado espaço diametral de um conjunto admissível $A$ se contém uma família completa de centros de $A$.

Teorema 2.2.11. Sejam X um espaço normado, A um subconjunto admissível de $X \in D$ espaço diametral de A. Então

$$
R_{D}(A)=D \cap A .
$$




\section{Demonstração :}

Como $D$ é espaço diametral de $A$ (i.e. $D$ contém uma família completa $S$ de centros de $A$ ), ele mesmo é uma família completa de centros, pois podemos estender a função $r: S \rightarrow[0,+\infty[$ para $D$ tomando, para cada $z \in D \backslash S$, um raio $r(z)$ tal que $A \subset \bar{B}_{r(z)}(z)$, que existe porque $A$ é limitado.

Portanto podemos escrever

$$
A=\bigcap_{y \in D} \bar{B}_{r(y)}(y) \text { onde } r: D \rightarrow[0,+\infty[
$$

Para $x \in \mathrm{A}$, temos que

$$
\|x-y\| \leq r(y) \text { para todo } y \in D,
$$

e pela proposição 1.1.4 sabemos que

$$
R_{D}(x)=D \cap\left(\bigcap_{y \in D} \bar{B}_{\|x-y\|}(y)\right)
$$

Logo ,

$$
R_{D}(A) \subseteq D \cap A .
$$

Reciprocamente, tomando $x \in D \cap A$ temos que

$$
R_{D}(x)=\{x\} \subseteq R_{D}(A)
$$

e portanto

$$
D \cap A \subseteq R_{D}(A)
$$

Teorema 2.2.12. Seja X um espaço de Banach de dimensão maior ou igual a 3. Então as seguintes afirmações são equivalentes:

(i) X é um espaço de Hilbert;

(ii) para quaisquer $x, y \in X$, tais que $x \perp y$, tem-se que $y \perp x$;

(iii) para todo hiperplano fechado $H$ de $X$ existe $x$ não nulo tal que $H \perp x$.

\section{Demonstração :}

Para uma demonstração deste teorema, ver [Jam47b], teoremas 1 e 5 . 
Teorema 2.2.13. Seja $X$ um espaço de Banach de dimensão maior ou igual a 3. Então as seguintes afirmações são equivalentes:

(i) X' é um espaço de Hilbert;

(ii) $A \cap D \neq \emptyset$ para todo subconjunto admissivel $A$ e todo espaço diametral $D$ de $A$;

(iii) para todo hiperplano fechado $G$ e todo $x$ de $X, R_{G}(x) \neq \emptyset$, ou seja, $D\left(R_{G}\right)=X$.

\section{Demonstração :}

(i) $\Rightarrow$ (ii)

Sejam $A$ um subconjunto admissível de $X$ e $D$ um espaço diametral de $A$. Como $X$ é um espaço de Hilbert, ele é reflexivo, e pelo lema 2.4 [Siño] (pag. 99), temos que $P_{D}(A) \neq \emptyset$.

Além disso, sabemos pela proposição 2.2.3 que $P_{D}(A)=R_{D}(A)$. Pelo teorema 2.2.11 também sabemos que $R_{D}(A)=A \cap D$.

Logo,

$$
A \cap D \neq \emptyset
$$

(ii) $\Rightarrow$ (iii)

Sejam $G$ um hiperplano fechado de $X$ e $x \in X$ quaisquer. Consideremos

$$
B_{G}(x)=\bigcap_{y \in G} \bar{B}_{\|x-y\|}(y) .
$$

É fácil ver que $B_{G}(x) \neq \emptyset$, pois $x \in B_{G}(x)$, e que, pela maneira que foi definido, $B_{G}(x)$ é admissivel e $G$ é espaço diametral de $B_{G}(x)$.

Então, utilizando a hipótese, temos que $G \cap B_{G}(x) \neq \emptyset$, que pela proposição 1.1.4 implica em

$$
R_{G}(x) \neq \emptyset \text {. }
$$

(iii) $\Rightarrow$ (i)

Pelo teorema 2.2 .12 basta provar que para todo hiperplano fechado $G$ existe $v \neq 0$ tal que $G \perp v$.

Seja $G$ um hiperplano de $X$. Tomemos $x \in X \backslash G$. Sabemos por hipótese que existe $g_{0} \in R_{G}(x)$, ou seja, pela proposição 2.2.2, $G \perp x-g_{0}$, oncle claramente $x-g_{0} \neq 0$. C'hamando $v=x-g_{0}$, o teorema está provado. 
Teorema 2.2.14. Seja X um espaço de Banach de dimensão maior ou igual a 3. Então são equivalentes:

(i) X é um espaço de Hilbert;

(ii) para todo subespaço fechado $G$ de $X$ tem-se $D\left(R_{G}\right)=X$.

\section{Demonstração :}

(i) $\Rightarrow$ (ii)

Imediata. Basta aplicar a proposiçào 2.2.3 e lembrar que em espaços de Hilbert a melhor aproximação existe e é única para todo subespaço fechado $G$ de $X$ e para todo $x \in X^{\prime}$.

(ii) $\Rightarrow$ (i)

Consideremos somente os hiperplanos fechados $H$ de $X$. Desta maneira temos que para todo hiperplano $H$ de $X$ existe $x \in X \backslash H$ tal que tem melhor coaproximação em $H$. Daí, pela proposição 2.2.2 temos que $H \perp\left(x-x_{0}\right)$, onde $x_{0} \in R_{H}(x)$.

Portanto, chamando $v=x-x_{0}$, provamos que existe $v \neq 0$ tal que $H \perp v$, e pelo teorema 2.2.12 temos que $X$ é um espaço de Hilbert.

Observação 2.2.15. Pelos teoremas 2.2.14 e 2.2.5 sabemos que só é possível afirmar a existência da melhor coaproximação para todo subespaço fechado e para todo ponto em espaços de Hilbert e em espaços de dimensão menor ou igual a 2.

Daremos agora alguns exemplos de não existência de melhor coaproximação.

Exemplo 2.2.16. Sejam $X=\left(\mathbb{R}^{3},\|\|_{\infty}\right)$ e $G$ o subespaço de dimensão 2 de equação $x_{1}+x_{2}+x_{3}=0$.

Temos que $R_{G}(x)=\emptyset$ para todo $x \in X \backslash G$. A justificação desta afirmação é geométrica (ver figura 2.3), notando que

$$
R_{G}(x)=G \cap\left(\bigcap_{g \in G}\left(\bar{B}_{\|g-x\|}(g)\right)\right) \subset G \cap\left(\bigcap_{i=1,2,3}\left(\bar{B}_{\left\|g_{i}-x\right\|}\left(g_{i}\right)\right)\right)=\emptyset .
$$




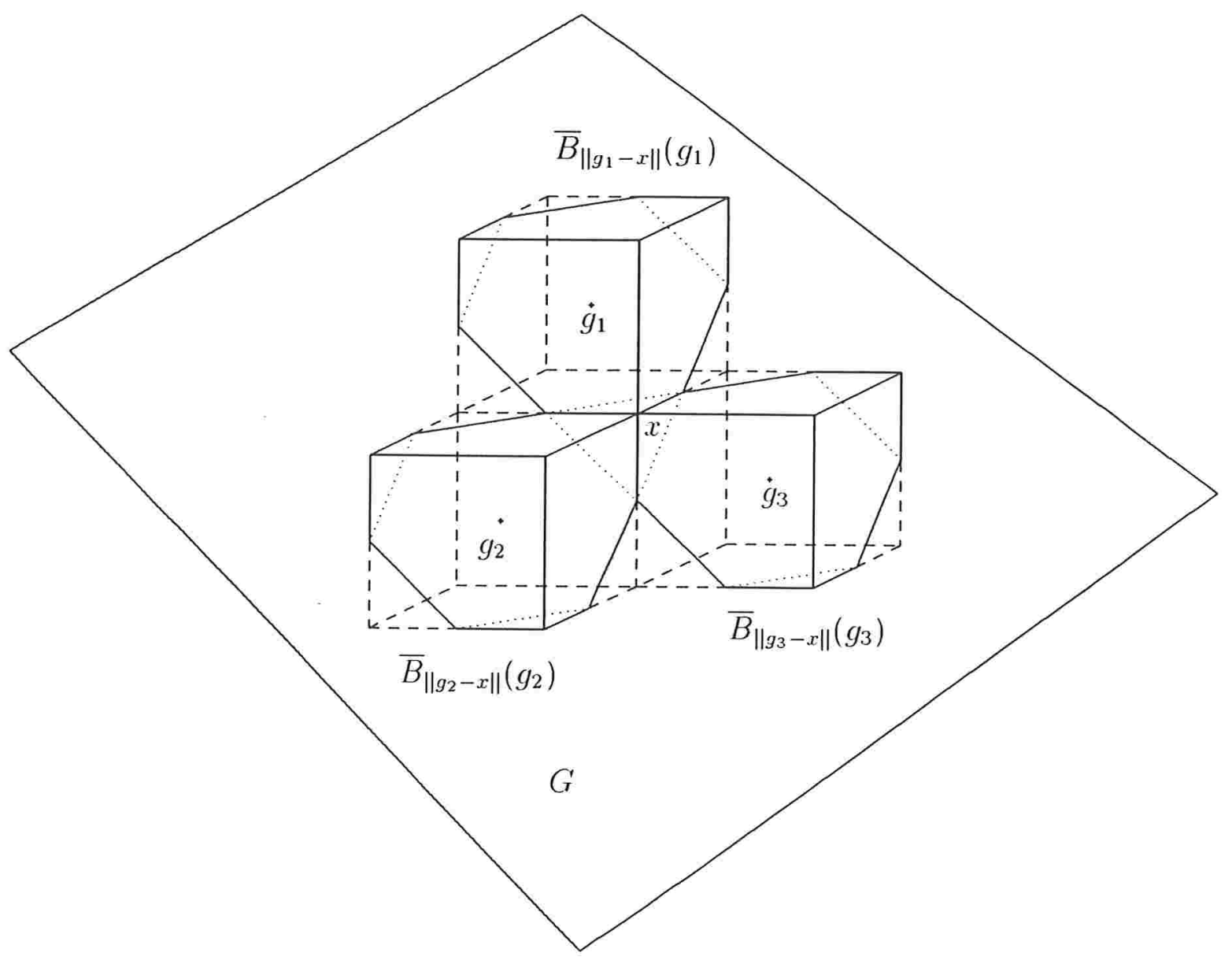

Figura 2.3.

Exemplo 2.2.17. Sejam $X=\left(C[0,1],\|\|_{\infty}\right), V=\{f \in X: f(0)=0\}$ e $a=a(t)=$ $1, t \in[0,1]$. Nestas condições vamos ver que $R_{V}(a)=\emptyset$. Suponhamos que existe $f_{0} \in V$ tal que

$$
\left\|f-f_{0}\right\|_{\infty} \leq\|f-a\|_{\infty} \text { para toda } f \in V .
$$

É fácil ver que existe $t_{0} \in[0,1]$ tal que $\left|f_{0}\left(t_{0}\right)\right|<1$, pois $f_{0}(0)=0$ e $f_{0}$ é contínua.

Basta então tomar $f$ da seguinte maneira:

$$
f(t)= \begin{cases}\frac{\left(3+f_{0}\left(t_{0}\right)\right) t}{t_{0}} & \text { se } 0 \leq t \leq t_{0} \\ 3+f_{0}\left(t_{0}\right) & \text { se } t_{0}<t \leq 1\end{cases}
$$


e terernos

$$
\left\|f-f_{0}\right\|_{\infty} \geq 3>2+f_{0}\left(t_{0}\right)=\|f-a\|_{\infty}
$$

Logo

$$
R_{V}(a)=\emptyset \text {. }
$$

A figura 2.4 ilustra este exemplo com $f_{0}\left(t_{0}\right)>0$, mas a mesma $f$ funciona se $f_{0}\left(t_{0}\right)<$ 0 .

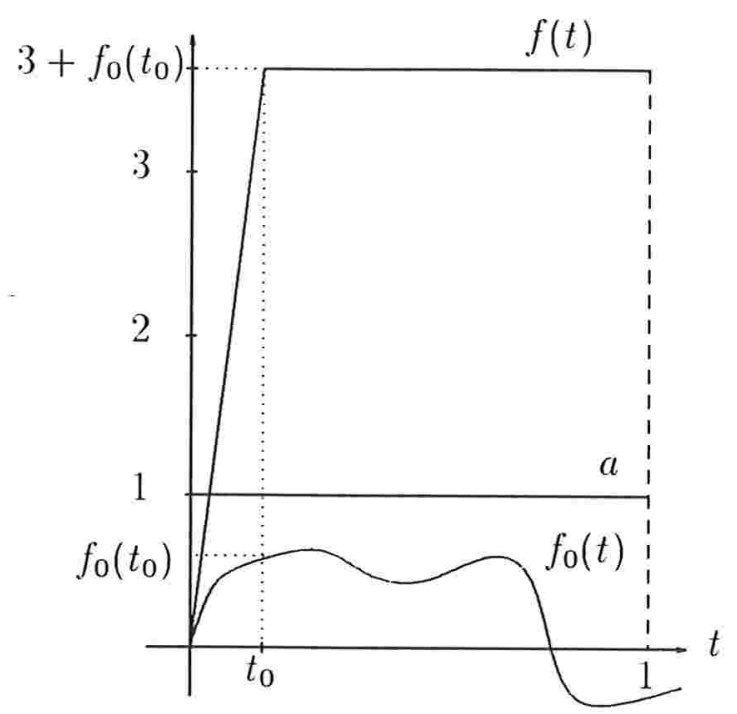

Figura 2.4. 


\subsection{Funções Convexas e Diferenciabilidade.}

Nesta seção estudaremos algumas propriedades das funções convexas e da diferencial de Gâteaux, que serão, conjuntamente com os resultados obtidos na seção 2.1, de uma grande ajuda para determinar, na seção 2.4, condições suficientes para a unicidade e linearidade da melhor coaproximação.

Definição 2.3.1. Sejam $Y$ um espaço vetorial e $D$ um subconjunto convexo de $Y$. Dizemos que $f: D \rightarrow \mathbb{R}$ é uma função convexa se para quaisquer $x$ e $y \in D$ e $0 \leq t \leq 1$,

$$
f[t x+(1-t) y] \leq t f(x)+(1-t) f(y) .
$$

Observação 2.3.2. Em um espaço normado $X$, a função $f(x)=\|x\|$ é uma função convexa definida em $X$.

Definição 2.3.3. Seja $f$ uma função com domínio um aberto $D$ de $X$. Para $x_{0} \in D$ e $x \in X$, definimos $d^{+} f_{x_{0}}(x)$ e $d^{-} f_{x_{0}}(x)$ por

$$
d^{+} f_{x_{0}}(x)=\lim _{t \rightarrow 0^{+}} \frac{f\left(x_{0}+t x\right)-f\left(x_{0}\right)}{t}, \text { se esse limite for um número real },
$$

e

$$
d^{-} f_{x_{0}}(x)=\lim _{t \rightarrow 0^{-}} \frac{f\left(x_{0}+t x\right)-f\left(x_{0}\right)}{t}, \text { se esse limite for um número real } .
$$

Chamaremos diferencial de Gâteaux da função $f$ em $x_{0}$ na direção $x$ a $d f_{x_{0}}(x)$ definida por

$$
d f_{x_{0}}(x)=\lim _{t \rightarrow 0} \frac{f\left(x_{0}+t x\right)-f\left(x_{0}\right)}{t}, \text { se esse limite for um número real } .
$$

Dizemos que uma função $f$ é Gâteaux diferenciável em $x_{0}$ se existe $d f_{x_{0}}(x)$ para todo $x \in X$.

Proposição 2.3.4. Seja $f$ uma função convexa definida num convexo aberto $D$ de $X$. Então a função $g(t)=\frac{f\left(x_{0}+t x\right)-f\left(x_{0}\right)}{t}$ é crescente à direita do zero $e$ limitada inferiormente e portanto $d^{+} f_{x_{0}}$ sempre existe. Dito de outra maneira. a função $g$ decresce até atingir seu limite quando $t \rightarrow 0^{+}$. De forma análoga, a função $m(t)=$ $-\frac{f\left(x_{0}-t x\right)-f\left(x_{0}\right)}{t}$ decresce à direita do zero e existe $d^{-} f_{x_{0}}$. 


\section{Prova :}

Se $x=0$, a afirmaçào é verdadeira. Consideremos então $x \in X^{Y} \backslash\{0\}$ e $x_{0} \in D$ fixados.

Como $D$ é aberto, temos que existe $\delta>0$ tal que $\bar{B}_{\delta}\left(x_{0}\right) \subset D$.

Tomemos $D_{1}=\bar{B}_{\delta}(0)$ (ver figura 2.5 ).

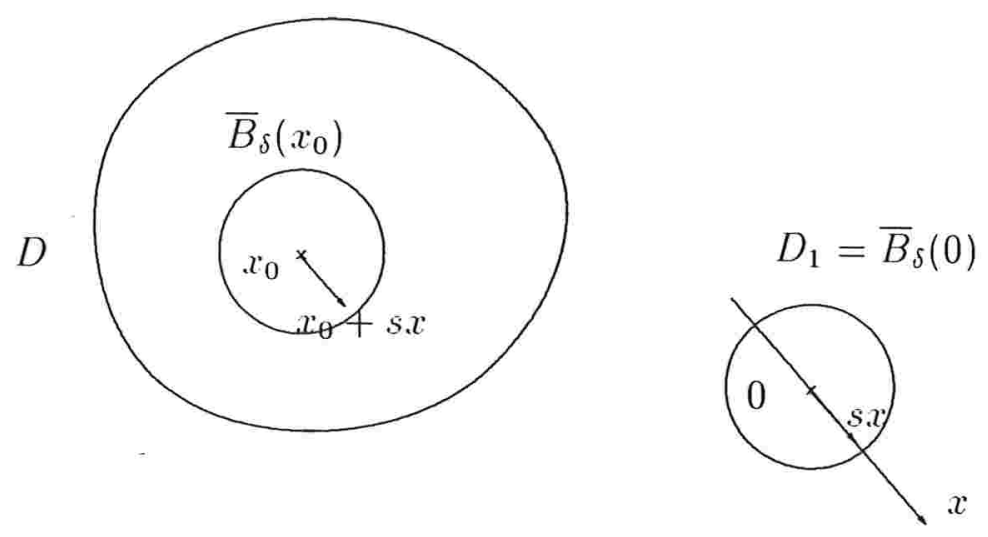

Figura 2.5.

Primeiramente provaremos o crescimento de $g$. Devemos provar então que

$$
\frac{f\left(x_{0}+t x\right)-f\left(x_{0}\right)}{t} \leq \frac{f\left(x_{0}+s x\right)-f\left(x_{0}\right)}{s} \text {, se } 0<t<s \text { e } s x \in D_{1} .
$$

Para isso, começaremos mostrando que $h(z)=f\left(z+x_{0}\right)$ é uma função convexa definida em $D_{1}$.

$$
\begin{gathered}
h(\alpha z+(1-\alpha) y)=f\left(\alpha z+(1-\alpha) y+x_{0}\right)=f\left(\alpha\left(z+x_{0}\right)+(1-\alpha)\left(y+x_{0}\right)\right) \leq \\
\leq \alpha f\left(z+x_{0}\right)+(1-\alpha) f\left(y-x_{0}\right)=\alpha h(z)+(1-\alpha) h(y) .
\end{gathered}
$$

Logo, $h$ é convexa.

Agora provaremos a desigualdade (2.8).

$$
\begin{gathered}
\frac{f\left(x_{0}+t x\right)-f\left(x_{0}\right)}{t}=\frac{h(t x)-h(0)}{t}=\frac{h\left(\left(1-\frac{t}{s}\right) 0+\frac{t}{s} s x\right)-h(0)}{t} \leq \\
\leq \frac{\left(1-\frac{t}{s}\right) h(0)+\frac{t}{s} h(s x)-h(0)}{t}=\frac{-h(0)+h(s x)}{s}=\frac{f\left(x_{0}+s x\right)-f\left(x_{0}\right)}{s} .
\end{gathered}
$$


Então $g(t)=\frac{f\left(x_{0}+t x\right)-f\left(x_{0}\right)}{t}$ é crescente à direita do zero. Portanto $g$ decresce quando $t \rightarrow 0^{+}$.

Aplicando o anterior em $-x$, temos que $m(t)=-\frac{f\left(x_{0}-t x\right)-f\left(x_{0}\right)}{t}$ é decrescente à direita do zero. Portanto $m$ cresce quando $t \rightarrow 0^{+}$.

Só resta provar que $g$ está limitada inferiormente e $m$ superiormente. Usando novamente a convexidade da $f$, temos que

$$
2 f\left(x_{0}\right) \leq f\left(x_{0}-t x\right)+f\left(x_{0}+t x\right)
$$

e então para $\delta \geq \frac{t}{\|x\|}>0$

$$
m(\delta) \leq \frac{-\left[f\left(x_{0}-t x\right)-f\left(x_{0}\right)\right]}{t} \leq \frac{f\left(x_{0}+t x\right)-f\left(x_{0}\right)}{t} \leq g(\delta)
$$

o que implica que a função $g(t)=\frac{f\left(x_{0}+t x\right)-f\left(x_{0}\right)}{t}$ está limitada inferiormente e a funçào $m(t)=\frac{-\left[f\left(x_{0}-t x\right)-f\left(x_{0}\right)\right]}{t}$ está limitada superiormente. Logo, concluem-se nossas afirmações.

Lema 2.3.5. Se $f$ é uma função convexa num aberto convexo $D$ de $X$, então $d^{+} f_{x_{0}}$ é sublinear, para todo $x_{0} \in D$.

\section{Prova :}

Precisamos mostrar, para cada $x_{0} \in D$,

(i) $d^{+} f_{x_{0}}(x+y) \leq d^{+} f_{x_{0}}(x)+d^{+} f_{x_{0}}(y)$ para quaisquer $x, y \in X$

$\mathrm{e}$

(ii) $d^{+} f_{x_{0}}(k x)=k d^{+} f_{x_{0}}(x)$ para todo $k \in \mathbb{R}^{+}$e todo $x \in X$.

Para (i). consideremos $x_{0} \in D, x, y \in X$ e $t>0$. Fazendo operações e usando o fato de que $f$ é convexa temos

$$
\begin{gathered}
f\left(x_{0}+t(x+y)\right)-f\left(x_{0}\right)=f\left(x_{0}+2 t\left(\frac{x+y}{2}\right)\right)-f\left(x_{0}\right)= \\
=f\left(\frac{1}{2}\left(x_{0}+2 t x\right)+\frac{1}{2}\left(x_{0}+2 t y\right)\right)-f\left(x_{0}\right) \leq \\
\leq \frac{f\left(x_{0}+2 t x\right)+f\left(x_{0}+2 t y\right)-2 f\left(x_{0}\right)}{2}=\frac{f\left(x_{0}+2 t x\right)-f\left(x_{0}\right)}{2}+\frac{f\left(x_{0}+2 t y\right)-f\left(x_{0}\right)}{2} .
\end{gathered}
$$


Dividindo por $t$ e fazendo o limite quando $t \rightarrow 0^{+}$obtemos

$$
\lim _{t \rightarrow 0^{+}} \frac{f\left(x_{0}+t(x+y)\right)-f\left(x_{0}\right)}{t} \leq \lim _{t \rightarrow 0^{+}} \frac{f\left(x_{0}+t x\right)-f\left(x_{0}\right)}{t}+\lim _{t \rightarrow 0^{+}} \frac{f\left(x_{0}+t y\right)-f\left(x_{0}\right)}{t},
$$

o que significa que

$$
d^{+} f_{x_{0}}(x+y) \leq d^{+} f_{x_{0}}(x)+d^{+} f_{x_{0}}(y)
$$

Para (ii), se $k \neq 0$, usando diretamente a definição para $x_{0} \in X, x \in X$ e $k \in \mathbb{R}^{+}$,

$$
\begin{aligned}
& d^{+} f_{x_{0}}(k x)=\lim _{t \rightarrow 0^{+}} \frac{f\left(x_{0}+k(t x)\right)-f\left(x_{0}\right)}{t}= \\
= & k \lim _{t \rightarrow 0^{+}} \frac{f\left(x_{0}+(k t) x\right)-f\left(x_{0}\right)}{k t}=k d^{+} f_{x_{0}}(x) .
\end{aligned}
$$

Se $k=0$ a igualdade é imediata.

Lema 2.3.6. Sejam $X$ um espaço normado e $p: X \rightarrow \mathbb{R}$ é uma função sublinear. Então

$$
p \text { é linear } \Longleftrightarrow p(-x)=-p(x) \text { para todo } x \in X \text {. }
$$

\section{Prova :}

$(\Rightarrow)$

Imediata.

$(\Leftarrow)$

Precisamos provar

(i) $p(a x)=a p(x)$ para todo $a \in \mathbb{R}$ e todo $x \in X$

e

(ii) $p(x+y)=p(x)+p(y)$, para quaisquer $x, y \in \mathbb{R}$.

Para (i), basta considerar $a<0$, e então temos que

$$
p(a x)=-a p(-x)=-a(-p(x))=a p(x) .
$$


Para (ii), basta provar que $p(x+y) \geq p(x)+p(y)$ para todo $x, y \in X$.

Como $p(x)=p(x+y-y) \leq p(x+y)+p(-y)=p(x+y)-p(y), \quad$ segue-se nosso resultado.

Proposição 2.3.7. Sejam $X$ um espaço normado e $f: X \rightarrow \mathbb{R}$ uma função convexa que seja Gâteaux diferenciável em $x_{0}$. Então $d f_{x_{0}}$ é linear.

Prova :

Sabendo por hipótese que $f$ é Gâteaux diferenciável em $x_{0}$, por definição

$$
\lim _{t \rightarrow 0} \frac{f\left(x_{0}+t x\right)-f\left(x_{0}\right)}{t} \text { existe para todo } x \in X .
$$

Trivialmente temos que $d f_{x_{0}}(x)=d^{+} f_{x_{0}}(x)$. Por outro lado temos, pelo lema 2.3.5, que $d^{+} f_{x_{0}}$ e sublinear e portanto $d f_{x_{0}}$ o é.

Além disso, é fácil ver que $d f_{x_{0}}(-x)=-d f_{x_{0}}(x)$. Logo, pelo lema 2.3.6, concluimos que $d f_{x_{0}}$ é linear.

Definição 2.3.8. Sejam $X$ um espaço normado. Dizemos que $X$ é suave se para todo $x \neq 0$ e todo $y \in X$, existe e é real o limite

$$
\lim _{t \rightarrow 0} \frac{\|x+t y\|-\|x\|}{t}
$$

isto é, a função norma é Gâteaux diferenciável em todos os pontos de $X \backslash\{0\}$.

Exemplo 2.3.9. Os espaços $X_{p}=\left(\mathbb{R}^{n},\|\|_{p}\right), 1<p<\infty$ são suaves.

Exemplo 2.3.10. $\left(C[0,1],\|\|_{\infty}\right)$ não é suave. Sejam $f(x)=1$ e $g(x)=\operatorname{sen}(2 \pi x)$ (ver figura 2.6).

É facil ver que

$$
\lim _{t \rightarrow 0} \frac{\|f+t g\|-\|f\|}{t} \text { nào existe, }
$$


pois

$$
\lim _{t \rightarrow 0^{+}} \frac{\|f+t g\|-\|f\|}{t}=1
$$

enquanto que

$$
\lim _{t \rightarrow 0^{-}} \frac{\|f+t g\|-\|f\|}{t}=-1 .
$$

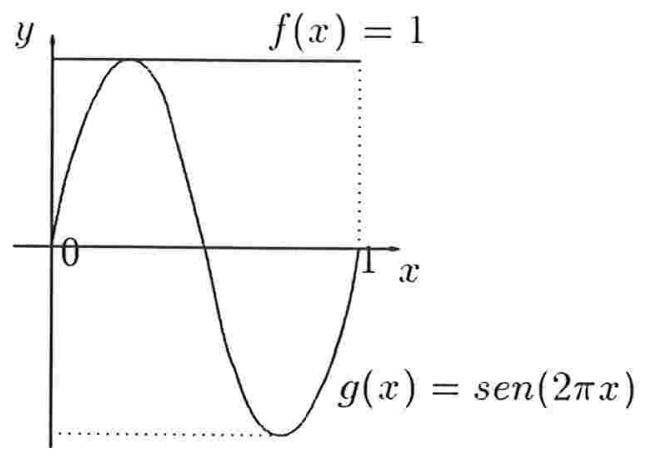

Figura 2.6. $\left(C[0,1],\|\|_{\infty}\right)$ não é suave. 


\subsection{Unicidade e Linearidade da Melhor Coaproxi- mação.}

Nesta seção estudaremos condições para a unicidade e linearidade da melhor coaproximação.

Lema 2.4.1. Sejam $X$ um espaço normado suave, $x \neq 0$ e y em $X$. Então

$$
x \perp y \Longleftrightarrow \lim _{t \rightarrow 0} \frac{\|x+t y\|-\|x\|}{t}=0 .
$$

Prova :

$(\Rightarrow)$

Como $x \perp y$, sabemos que $\|x\| \leq\|x+t y\|$ para todo $t \in \mathbb{R}$. Daí

$$
\lim _{t \rightarrow 0^{+}} \frac{\|x+t y\|-\|x\|}{t} \geq 0 \text { e } \lim _{t \rightarrow 0^{-}} \frac{\|x+t y\|-\|x\|}{t} \leq 0
$$

donde

$$
\lim _{t \rightarrow 0} \frac{\|x+t y\|-\|x\|}{t}=0,
$$

pois, como $X$ é suave, existe esse último limite e é igual aos outros dois. $(\Leftarrow)$

Sabemos que

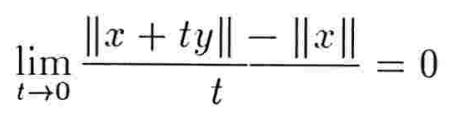

e portanto

$$
\lim _{t \rightarrow 0^{ \pm}} \frac{\|x+t y\|-\|x\|}{t}=0 .
$$

Logo, como a função norma é convexa, pela proposição 2.3.4,

$$
\|x+t y\|-\|x\| \geq 0 \text { se } t \geq 0 \quad \text { e } \quad\|x+t y\|-\|x\| \geq 0 \text { se } t \leq 0 .
$$

Portanto

$$
x \perp y .
$$


Teorema 2.4.2. Seja X um espaço normado. Então são equivalentes:

(i) X'é suave;

(ii) a ortogonalidade é aditiva à direita;

(iii) dados $x, y$ em $X, x$ não nulo, existe um único $a \in \mathbb{R}$ tal que $x \perp a x+y$.

\section{Demonstração :}

(i) $\Rightarrow$ (ii)

Sejam $x, y$ e $z$ elementos de $X$ tais que $x \perp y$ e $x \perp z$. Devemos provar que $x \perp(y+z)$. Como para $x=0$ a afirmação é óbvia, consideraremos $x \neq 0$.

Pelo lema 2.4.1 $(\Rightarrow)$, sabemos que

$$
\lim _{t \rightarrow 0} \frac{\|x+t y\|-\|x\|}{t}=0 \text { e } \lim _{t \rightarrow 0} \frac{\|x+t z\|-\|x\|}{t}=0 .
$$

Escrito de outra maneira, tomando $f=\|\|$,

$$
d f_{x}(y)=0 \quad \text { e } \quad d f_{x}(z)=0 .
$$

Pelo corolário 2.3.7 temos que

$$
d f_{x}(y)+d f_{x}(z)=d f_{x}(y+z)=0,
$$

ou seja,

$$
\lim _{t \rightarrow 0} \frac{\|x+t(y+z)\|-\|x\|}{t}=0 .
$$

Portanto, pelo lema 2.4.1 $(\Leftarrow)$,

$$
x \perp(y+z) .
$$

(ii) $\Rightarrow$ (iii)

Sejam $x \neq 0$ e $y$ em $X$ quaisquer. Pela proposição 2.1.8, sabemos que existem $A$ e $B$ em $\mathbb{R}$ tais que $x \perp A x+y$ e $x \perp B x+y$, onde $A$ e $B$ são respectivamente, o menor e o maior dos números $a$ tais que $x \perp a x+y$.

Logo, pela homogeneidade da ortogonalidade e usando a hipótese, conclue-se que

$$
x \perp(A-B) x,
$$


e portanto,

$$
A=B \text {, }
$$

donde existe um único $a \in \mathbb{R}$ tal que $x \perp a x+y$.

(iii) $\Rightarrow$ (i)

Sejam $x \neq 0$ e $y$ em $X$ quaisquer. Devemos provar que $\lim _{t \rightarrow 0} \frac{\|x+t y\|-\|x\|}{t}$ existe, ou ainda, que $\lim _{t \rightarrow 0^{+}} \frac{\|x+t y\|-\|x\|}{t}=\lim _{t \rightarrow 0^{-}} \frac{\|x+t y\|-\|x\|}{t}$. pois esses dois limites existem (proposição 2.3.4).

Calculemos o primeiro:

$$
\begin{gathered}
\lim _{t \rightarrow 0^{+}} \frac{\|x+t y\|-\|x\|}{t}=\lim _{t \rightarrow 0^{+}} \frac{1}{t}(\|x+t y\|-\|x\|)= \\
=\lim _{n \rightarrow+\infty} n\left(\left\|x+\frac{1}{n} y\right\|-\|x\|\right)=\lim _{n \rightarrow+\infty}(\|n x+y\|-\|n x\|) .
\end{gathered}
$$

Mas esse último limite é conhecido e igual a $-A\|x\|$, onde $A$ é o menor dos $a \in \mathbb{R}$ que satisfazem $x \perp a x+y$.

Logo,

$$
\lim _{t \rightarrow 0^{+}} \frac{\|x+t y\|-\|x\|}{t}=-A\|x\| .
$$

Analogamente, se prova que

$$
\lim _{t \rightarrow 0^{-}} \frac{\|x+t y\|-\|x\|}{t}=-B\|x\|
$$

onde $B$ é o maior dos $a \in \mathbb{R}$ que satisfazem $x \perp a x+y$. Como por hipótese $A=B$, deduz-se que $X$ é suave.

Teorema 2.4.3. $X$ é suave se e somente se $R_{G}(y)$ é unitário para todo $y \in D\left(R_{G}\right)$ e todo subespaço $G$ de $X$.

\section{Demonstração :}

$(\Rightarrow)$

Sejam $x$ em $X, g_{1}$ e $g_{2}$ em $R_{G}(x)$. Mostraremos que $g_{1}=g_{2}$.

Como $g_{1} \in R_{G}(x)$ e $g_{2} \in R_{G}(x)$ então $G \perp\left(x-g_{1}\right)$ e $G \perp\left(x-g_{2}\right)$. Logo, pelo teorema 2.4 .2 temos que $G \perp\left(g_{1}-g_{2}\right)$. Daí obtemos que $g_{1}-g_{2}=0$, pois $g_{1}-g_{2} \in G$ porque $G$ é subespaço. 
$(\Leftarrow)$

Suponhamos que $X$ não é suave. Entào existem $x \neq 0$ e $y$ em $X$, tais que

$$
-A\|x\|=\lim _{t \rightarrow 0^{+}} \frac{\|x+t y\|-\|x\|}{t} \neq \lim _{t \rightarrow 0^{-}} \frac{\|x+t y\|-\|x\|}{t}=-B\|x\| .
$$

Donde, pelo lema 2.4.1, temos que $x \perp y+A x$ e $x \perp y+B x$.

Portanto, tomando $[x]=G$, concluimos que $G \perp y-(-A x)$ e $G \perp y-(-B x)$ e daí

$$
-A x \in R_{G}(y) \text { e }-B x \in R_{G}(y) .
$$

Logo, $R_{G}(y)$ não é unitário, o que é absurdo.

Teorema 2.4.4. Se X' é um espaço normado suave e $G$ é um subespaço de $X$, então

(i) para todo $x \in X$, se não for vazio. $R_{G}(x)$ é unitário;

(ii) $D=D\left(R_{G}\right)$ é um subespaço de $X$;

(iii) $R_{G}: D \rightarrow G$ é linear (considerado como função).

\section{Demonstração :}

(i) Foi provado no teorema 2.4.3.

(ii) Sejam $x, y \in D\left(R_{G}\right)$. Pela proposição 2.2.2, existem $x_{0}, y_{0} \in G$ tais que $G \perp x-x_{0}$ e $G \perp y-y_{0}$. Pelo teorema 2.4.2, $G \perp(x+y)-\left(x_{0}+y_{0}\right)$.

Novamente pela proposição $2.2 .2, x+y \in D\left(R_{G}\right)$ pois $x_{0}+y_{0} \in G$.

Para $\lambda \in \mathbb{R}$, pelo lema 2.1.5, $G \perp \lambda x-\lambda x_{0}$ e, pela proposição $2.2 .2, \lambda x \in D\left(R_{G}\right)$ pois $\lambda x_{0} \in G$.

(iii) Imediata pela demonstração (ii). 


\section{Capítulo 3}

\section{Hipercírculos de Chebyshev e Coaproximação}

O objetivo deste capítulo é caracterizar espaços de Hilbert a partir de espaços reflexivos usando a melhor coaproximação. Para chegar a ele, primeiramente estudaremos algumas propriedades dos hipercírculos e centros de Chebyshev, as quais têm uma importância relevante no desenvolvimento do capítulo.

\subsection{Hipercírculos e Centros de Chebyshev}

Nesta seção daremos os conceitos e algumas propriedades, tanto dos hipercírculos como dos centros de Chebyshev. Os resultados serão muito úteis na seção 3.2 , na qual acharemos uma caracterização para espaços de Hilbert a partir dos espaços reflexivos.

Definição 3.1.1. Sejam $A$ e $V$ subconjuntos não vazios de $X, A$ limitado. Consideramos $F_{A}: X \rightarrow \mathbb{R}^{+}$dada por

$$
F_{A}(x)=\sup _{y \in A}\{\|x-y\|\}, x \in X .
$$

Chamamos Raio de Chebyshev de $A$, denotado por $r(A)$, a

$$
r(A)=\inf _{x \in X} F_{A}(x) .
$$


Chamamos Raio de Chebyshev de A com respeito a $V$, denotado por $r_{V}(A)$, a

$$
r_{V}(A)=\inf _{x \in V} F_{A}(x)
$$

Um elemento $x_{0}$ de $X$ é chamado centro de $A$ se $F_{A}\left(x_{0}\right)=r(A)$. Analogamente, um ponto $y_{0}$ de $V$ é chamado centro de $A$ com respeito $a V$, se $F_{A}\left(y_{0}\right)=r_{V}(A)$. Denotaremos $E(A)$ o conjunto de centros de $A$ e $E_{V}(A)$ o conjunto de centros de $A$ com respeito a $V$.

Definição 3.1.2. Sejam $V$ um subespaço fechado de um espaço normado $X, a \in X$ e $s \in \mathbb{R}$ tais que $d(a, V)=d<s$. Definimos $s$-hipercírculo de $V$ como

$$
C_{s}=\bar{B}_{s}(a) \cap V=\{v \in V:\|v-a\| \leq s\} .
$$

Denotaremos $r_{s}=r_{V}\left(C_{s}\right)=\inf _{y \in V}\left[\sup _{x \in C_{s}}\|x-y\|\right]$.

Observação 3.1.3. É fácil ver que $\inf _{y \in C_{s}}\|a-y\|=\inf _{y \in V}\|a-y\|=d$. Basta observar que se $y \in V \backslash C_{s},\|a-y\|>s \geq \inf _{z \in C_{s}}\|a-z\|$.

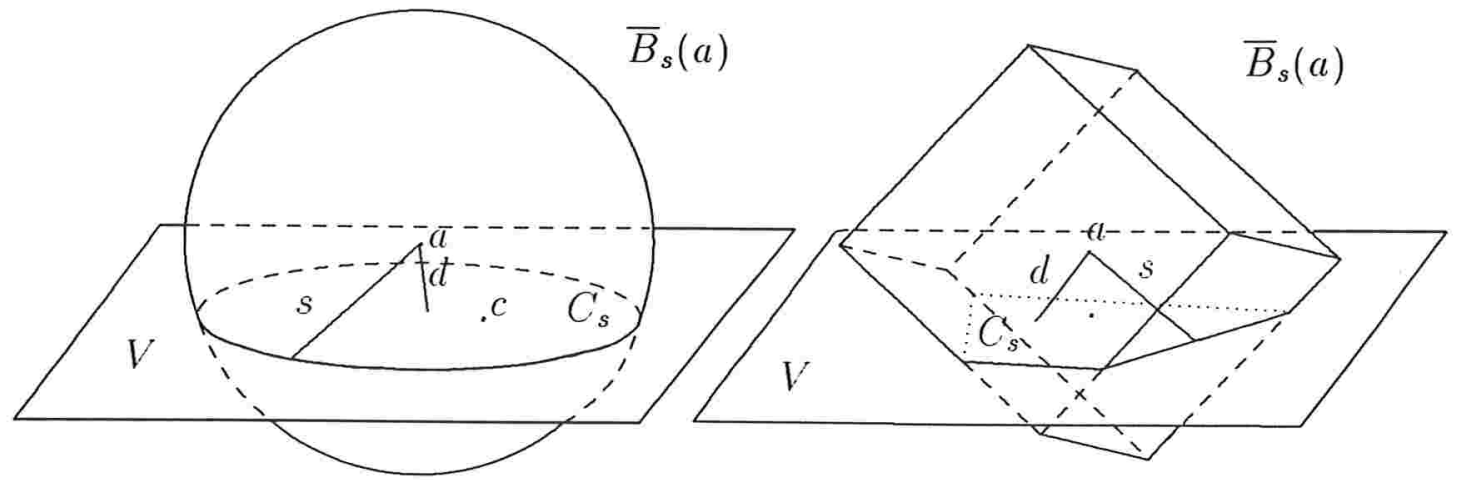

Figura 3.1.

A partir daquí consideramos $X$ um espaço de Banach real, e fixamos um subespaço fechado nào trivial $V$ de $X$ e $a \in X \operatorname{com} d(a, V)=d$.

Proposição 3.1.4. Se $s>d$, então

$$
c \in E_{V}\left(C_{s}\right) \Longleftrightarrow\left\{\begin{array}{l}
c \in V \\
\|c-x\| \leq\|a-x\|+r_{s}-s, \quad \forall x \in C_{s} .
\end{array}\right.
$$




\section{Prova :}

$(\Leftarrow)$

Sabemos da hipótese que para todo $x \in C_{s}$

$$
\|c-x\| \leq\|a-x\|+r_{s}-s .
$$

Tomamos o supremo em $x \in C_{s}$ e obtemos

$$
\sup _{x \in C_{s}}\|c-x\| \leq \sup _{x \in C_{s}^{\prime}}\left\{\|a-x\|+r_{s}-s\right\} .
$$

Como $\sup _{x \in C_{s}}\|a-x\| \leq s$, pois $C_{s} \subset \bar{B}_{s}(a)$, temos que

$$
\sup _{x \in C_{s}}\|c-x\| \leq r_{s} .
$$

Mas $r_{s}=\inf _{y \in V}\left[\sup _{x \in C_{s}}\|x-y\|\right] \leq \sup _{x \in C_{s}}\|c-x\|$, porque $c \in V$.

Daí, $\sup _{x \in C_{s}}\|c-x\|=r_{s}$ e portanto

$$
c \in E_{V}\left(C_{s}\right)
$$

$(\Rightarrow)$

Separemos em dois casos: (i) $x=c$ e (ii) $x \neq c$.

(i) $x=c$

Como $r_{s}=\sup _{z \in C_{s}}\|z-c\|$, temos que para todo $z \in C_{s}, r_{s} \geq\|z-c\|$, em particular para $z$ na fronteira de $C_{s}$, onde, além disso, sabemos que $\|a-z\|=s$.

Por outro lado, pela desigualdade triangular temos que para todo $\approx \in X$ $\|z-c\| \geq\|a-z\|-\|a-c\|$.

Em resumo temos que $r_{s} \geq s-\|a-c\|$, ou $\|a-c\|+r_{s}-s \geq 0$.

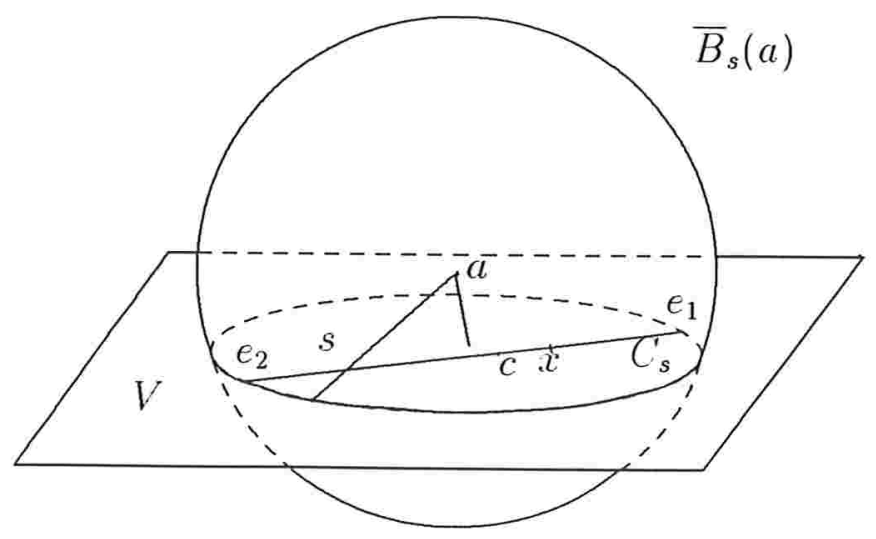

Figura 3.2. 
(ii) $x \neq c$.

Se $x \neq c$, a reta $c x$ corta a fronteira de $C_{s}$ em dois pontos distintos $e_{1}$ e $e_{2}$ pertencentes a $C_{s}$, porque $X$ é completo, $V$ é fechado e $C_{s}$ é convexo, limitado e fechado. Eles podem ser expressos assim:

$e_{1}=\lambda_{1} c+\left(1-\lambda_{1}\right) x$, e $e_{2}=\lambda_{2} c+\left(1-\lambda_{2}\right) x$, e é simples ver que $\lambda_{1}$ e $\lambda_{2}$ não podem ser positivos ao mesmo tempo. Vamos supor que $\lambda_{1}$ não é positivo.

Sabemos que $\left\|c-e_{1}\right\| \leq r_{s}$, pois $c$ é centro de $C_{s}$ e $\epsilon_{1} \in C_{s}$, e então

$$
\left\|c-\lambda_{1} c-\left(1-\lambda_{1}\right) x\right\|=\left(1-\lambda_{1}\right)\|c-x\| \leq r_{s}=r_{s}-s+s=r_{s}-s+\left\|a-e_{1}\right\| .
$$

Por outro lado,

$$
\left\|a-e_{1}\right\|=\left\|a-\lambda_{1} c-\left(1-\lambda_{1}\right) x\right\| \leq\|a-x\|-\lambda_{1}\|c-x\|,
$$

e portanto

$$
\left(1-\lambda_{1}\right)\|c-x\| \leq r_{s}-s+\|a-x\|-\lambda_{1}\|c-x\| .
$$

Logo

$$
\|c-x\| \leq r_{s}-s+\|a-x\| .
$$

Como os conjuntos $E$ e $E_{V}$ podem ser vazios, vamos definir "quase centros" de modo que seja possível garantir sua existência.

Definição 3.1.5. Nas condições da definição 3.1.1, consideramos $\varepsilon>0$. Um elemento $c$ de $X$ é chamado $\varepsilon$-quase centro de $A$ se

$$
\sup _{x \in A}\|c-x\| \leq r(A)+\varepsilon .
$$

Analogamente, um ponto $c$ de $V$ é chamado $\varepsilon$-quase centro de $A$ com respeito a $V$ se

$$
\sup _{x \in A}\|c-x\| \leq r_{V}(A)+\varepsilon .
$$

Denotaremos $E^{\varepsilon}(A)$ o conjunto de $\varepsilon$-quase centros de $A$ e $E_{V}^{\varepsilon}(A)$ o conjunto de E-quase centros de $A$ com respeito a $V$, ou seja,

$$
E^{\varepsilon}(A)=\left\{c \in X: \sup _{v \in A}\|c-v\| \leq r(A)+\varepsilon\right\}
$$

e

$$
E_{V}^{\varepsilon}(A)=\left\{c \in V: \sup _{v \in A}\|c-v\| \leq r_{V}(A)+\varepsilon\right\} .
$$


Proposição 3.1.6. Se $s>d$ e $C_{s}$ é o s-hipercírculo de $V$, então, para qualquer $\varepsilon>0$,

$$
c \in E_{V}^{\varepsilon}\left(C_{s}\right) \Longleftrightarrow\left\{\begin{array}{l}
c \in V \\
\|c-x\| \leq\|a-x\|+r_{s}-s+\varepsilon \forall x \in C_{s} .
\end{array}\right.
$$

Prova :

Análoga à proposição 3.1.4.

Proposição 3.1.7. Se $s>d$, vale que

$$
s-d \leq r_{s} \leq s+d .
$$

Prova :

Por definição e pela desigualdade triangular, temos

$$
\begin{gathered}
r_{s}=\inf _{y \in V}\left[\sup _{x \in C_{s}^{\prime}}\|x-y\|\right] \leq \sup _{x \in C_{s}}\|x-y\| \\
\leq \sup _{x \in C_{s}}\{\|x-a\|+\|a-y\|\}=\|a-y\|+\sup _{x \in C_{s}}\|x-a\|= \\
=\|a-y\|+s, \text { para todo } y \in V .
\end{gathered}
$$

Logo,

$$
r_{s} \leq d+s
$$

Dado $\varepsilon>0$, sejam $c \in E_{V}^{\varepsilon}\left(C_{s}\right)$ e $x$ qualquer elemento de $C_{s}$. Então

$$
r_{s} \geq s-\varepsilon+\|c-x\|-\|a-x\| \geq s-\varepsilon-\|a-x\| .
$$

Daí, como $x$ é qualquer, pela observação $3.1 .3, r_{s} \geq s-d-\varepsilon$ para todo $\varepsilon>0$, o que implica

$$
r_{s} \geq s-d
$$

Os exemplos a seguir mostram que podem ocorrer as igualdades na proposição 3.1.7. 
Exemplo 3.1.8. Consideremos $X=\left(C[0,1],\|\|_{\infty}\right), V=\{f \in X: f(0)=0\}$ e $a=a(t)=1, t \in[0,1]$. Como $d(a, V)=1$, devemos tomar $s=d+\varepsilon=1+\varepsilon, \varepsilon>0$. Seja $f \in V$ qualquer. Se $f \equiv 0$, é imediato que

$$
\sup _{y \in C_{s}^{\prime}}\|f-y\|=\sup _{y \in C_{s}}\|y\|=2+\varepsilon .
$$

Suponhamos então $f \not \equiv 0$ e consideremos dois casos:

(a) existe algum $t_{0} \in(0,1]$ tal que $f\left(t_{0}\right)<0$,

(b) $f(t) \geq 0$ para todo $t \in[0,1]$.

(a) Neste caso escolhemos a função $y_{t_{0}} \in C_{s}$ dada por

$$
y_{t_{0}}(t)= \begin{cases}\frac{2+\varepsilon}{t_{0}} t & \text { se } 0 \leq t \leq t_{0} \\ 2+\varepsilon & \text { caso contrário } .\end{cases}
$$

Logo,

$$
\sup _{y \in C_{s}}\|f-y\|>2+\varepsilon
$$

(b) Agora temos que $f \not \equiv 0$ e que $f(t) \geq 0$ para todo $t \in[0,1]$. Seja $\delta<m$ qualquer, onde $m=\max _{t \in[0,1]}\{f(t)\}$. Pela continuidade de $f$, temos que existe $t_{\delta} \in(0,1]$ tal que $f\left(t_{\delta}\right)=\delta$. Escolhemos $y_{\delta} \in C_{s}$ dada por

$$
y_{\delta}(t)= \begin{cases}\frac{2+\varepsilon}{t_{\delta}} t & \text { se } 0 \leq t \leq t_{\delta} \\ 2+\varepsilon & \text { caso contrário. }\end{cases}
$$

Portanto, $\left\|f-y_{\delta}\right\| \geq 2+\varepsilon-\delta$ e como $\delta$ é qualquer, concluimos que

$$
\sup _{y \in C_{s}}\|f-y\| \geq 2+\varepsilon \text {. }
$$

Em resumo, por (3.4), (3.5) e (3.6), temos que para todo $f \in V$ vale

$$
\sup _{y \in C_{s}}\|f-y\| \geq 2+\varepsilon
$$


e pela proposição anterior deduz-se que

$$
r_{s}=\inf _{f \in V}\left[\sup _{y \in C_{s}}\|f-y\|\right]=2+\varepsilon .
$$

Assim,

$$
r_{s}=s+d
$$
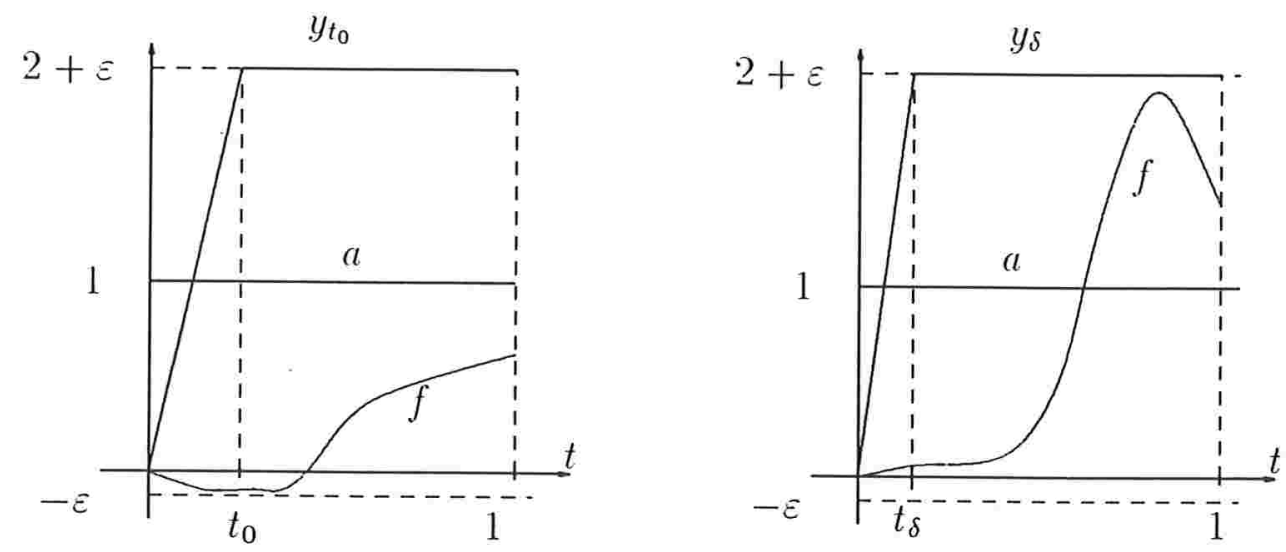

\section{Figura 3.3.}

Exemplo 3.1.9. Consideremos agora $X=\left(\mathbb{R}^{2},\|\|_{\infty}\right), V=\left\{\left(x_{1}, x_{2}\right) \in X: x_{1}=\right.$ $\left.-x_{2}\right\}$ e $a=(1,1)$.

Novamente $d=1$, e tomamos $s=1+\varepsilon, \varepsilon>0$, só que neste caso $r_{s}=\varepsilon$, portanto

$$
r_{s}=s-d .
$$

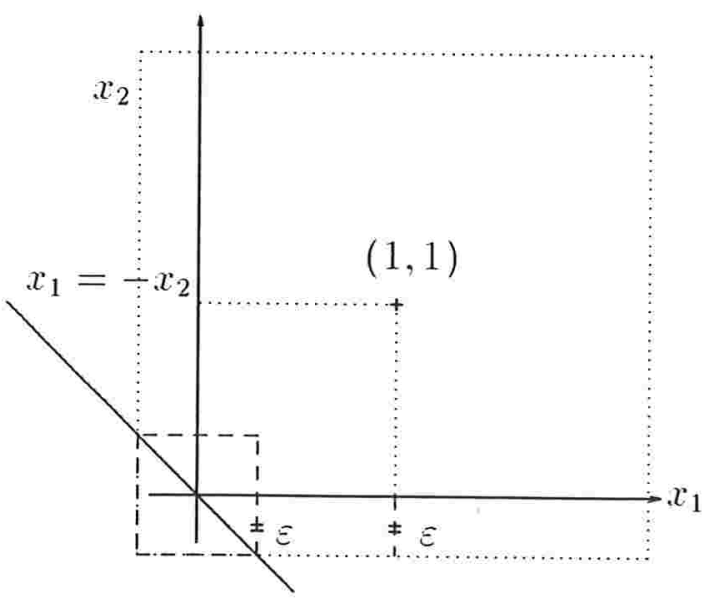

Figura 3.4. 
Corolário 3.1.10. Se $s>d, C_{s}$ é o s-hipercírculo de $V$ e $c \in E_{V}\left(C_{s}\right)$, valem

1. Se $\left\{x_{n}\right\}$ é uma seqüência em $C_{s}$ e $\left\|x_{n}-c\right\| \stackrel{n}{\rightarrow} r_{s}$, então $\left\|a-x_{n}\right\| \stackrel{n}{\rightarrow} s$.

2. $\|c-a\| \leq 2 d+r_{s}-s$.

3. Se $s \geq 2 d+r_{s}-s$ então $c \in C_{s}$.

4. Se $0 \in C_{s}$ então $\|c\| \leq\|a\|+r_{s}-s$.

5. Se $v_{0} \in P_{V}(a)$ então $\left\|c-v_{0}\right\| \leq d+r_{s}-s$.

Prova :

1. Sabemos que $c \in E_{V}\left(C_{s}\right)$ e então

$$
\|c-x\| \leq\|a-x\|+r_{s}-s \text {, para todo } x \in C_{s} .
$$

Portanto, como $x_{n} \in C_{s}$, temos $s-\left\|a-x_{n}\right\| \leq r_{s}-\left\|c-x_{n}\right\|$ e $\left\|a-x_{n}\right\| \leq s$, donde, $0 \leq s-\left\|a-x_{n}\right\| \leq r_{s}-\left\|c-x_{n}\right\|$, para todo $n \in \mathbb{N}$. Tomando $n \rightarrow \infty$, está provada a afirmação.

2. Para todo $x \in C_{s}$, como

$$
\|c-a\|=\|c-x+x-a\| \leq\|c-x\|+\|x-a\|,
$$

temos que

$$
\|c-a\| \leq\|a-x\|+r_{s}-s+\|x-a\|=2\|a-x\|+r_{s}-s .
$$

Logo, pela observação 3.1 .3 , tomando o ínfimo em $x \in C_{s}$, obtemos o resultado desejaclo.

3. É imediato pela parte anterior.

4. Imediato. Basta substituir $x=0$ na proposição 3.1.4.

5. Seja $v_{0} \in P_{V}(a)$. Então $v_{0} \in C_{s}$, pois $d=\left\|v_{0}-a\right\|<s$, e portanto

$$
\left\|c-v_{0}\right\| \leq\left\|a-v_{0}\right\|+r_{s}-s=d+r_{s}-s .
$$


Proposição 3.1.11. Se $X$ é um espaço de Hilbert e $s>d$, então

$$
r_{s}=\sqrt{s^{2}-d^{2}}
$$

Prova :

Sabemos que $P_{V}(a)$ é unitário. Seja $P_{V}(a)=\left\{h_{0}\right\}$.

$(\leq)$

Mostraremos que $r_{s}^{2} \leq s^{2}-d^{2}$ :

$$
r_{s}^{2} \leq \sup _{x \in C_{s}^{\prime}}\left\|h_{0}-x\right\|^{2}=\sup _{x \in C_{s}}\left(\|x-a\|^{2}-d^{2}\right) \leq s^{2}-d^{2} .
$$

$(\geq)$

Seja $\delta$ o diâmetro de $C_{s}$ e sejam $\alpha$ e $\beta$ os pontos de corte da fronteira de $C_{s}$ com uma reta qualquer pelo ponto $h_{0}$.

Então temos

$$
\|\alpha-\beta\|=2 \sqrt{s^{2}-d^{2}} \text {, pois o triângulo }(a \alpha \beta) \text { é isósceles . }
$$

Portanto

$$
\delta \geq 2 \sqrt{s^{2}-d^{2}}
$$

Consideremos $\varepsilon>0$ e $y \in E_{V}^{s}\left(C_{s}\right)$. Então para quaisquer $x, z \in C_{s}$ vale que

$$
\|z-x\| \leq\|z-y\|+\|x-y\| \leq \sup _{z \in C_{s}}\|z-y\|+\sup _{x \in C_{s}}\|x-y\| \leq 2 r_{s}+2 \varepsilon .
$$

Tomando o supremo em $x, z \in C_{s}$ obtemos

$$
\delta \leq 2 r_{s}+2=.
$$

Daí, como é é qualquer,

$$
r_{s} \geq \frac{1}{2} \delta
$$

e portanto

$$
r_{s} \geq \sqrt{s^{2}-d^{2}}
$$


Proposição 3.1.12. Se X é um espaço de Hilbert e s $>$ d então o único centro de $C_{s}$ é $h_{0}$, onde $P_{V}(a)=\left\{h_{0}\right\}$.

\section{Prova :}

Para provar que $h_{0}$ é centro de $C_{s}$, pela proposiçào 3.1 .4 basta mostrar que

$$
\left\|h_{0}-x\right\| \leq\|a-x\|+r_{s}-s \quad \forall x \in C_{s} .
$$

Pela proposição 3.1 .7 sabemos que $\left|r_{s}-s\right| \leq d$ e como $\|a-x\| \geq d$, vamos provar que

$$
\left\|h_{0}-x\right\|^{2} \leq\left(\|a-x\|+r_{s}-s\right)^{2} \text { para todo } x \in C_{s},
$$

o que por Pitágoras significa

$$
\|a-x\|^{2}-d^{2} \leq\|a-x\|^{2}-2\left(s-r_{s}\right)\|a-x\|+\left(s-r_{s}\right)^{2},
$$

ou ainda,

$$
\left(s-r_{s}\right)^{2}-2\left(s-r_{s}\right)\|a-x\|+d^{2} \geq 0 \text { para todo } x \in C_{s} .
$$

Mas pela proposição 3.1 .11 sabemos que

$$
d^{2}=s^{2}-r_{s}^{2},
$$

donde

$$
\left(s-r_{s}\right)^{2}-2\left(s-r_{s}\right) s+d^{2}=0
$$

e também

$$
s-r_{s} \geq 0 .
$$

Como $\|a-x\| \leq s$ para todo $x \in C_{s}$, obtemos o resultado desejado.

Agora provaremos que $h_{0}$ é o único centro de $C_{s}$. Suponhamos que exista $c$, centro de $C_{s}$ tal que $c \neq h_{0}$. Seja então a reta $r=\left\{\alpha h_{0}+(1-\alpha) c: \alpha \in \mathbb{R}\right\}$. A reta $r$ corta a fronteira de $C_{s}$ em dois pontos distintos $e_{1}$ e $e_{2}$ pertencentes a $C_{s}$ porque $X$, fechado é completo, $V$ é fechado e $C_{s}$ é convexo, limitado e fechado. 


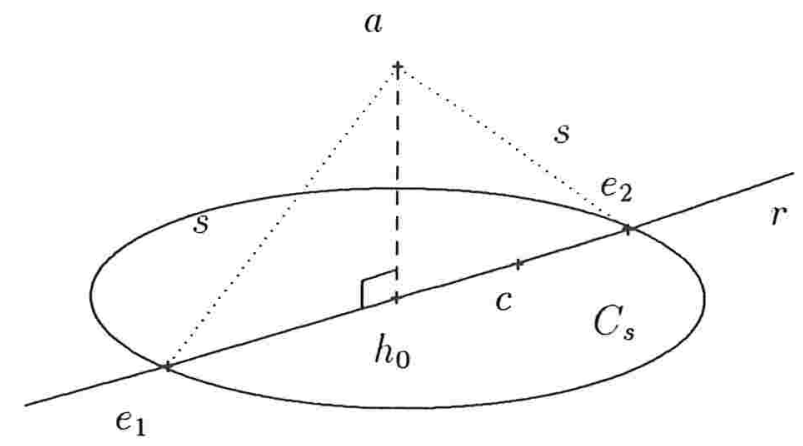

\section{Figura 3.5.}

É claro, pela proposição 3.1.11, que $e_{1} \neq h_{0} \neq e_{2}$ pois $\left\|e_{1}-h_{0}\right\|=\left\|e_{2}-h_{0}\right\|=r_{s}$. Além disso, $c$ está entre $h_{0}$ e $e_{1}$ ou $e_{2}$ estritamente.

Suponhamos que $c$ está entre $e_{2}$ e $h_{0}, \operatorname{logo}\left\|c-e_{1}\right\|>r_{s}$, o que é absurdo. Resulta análogo se $c$ está entre $e_{2}$ e $h_{0}$. 


\subsection{Uma Caracterização para Espaços de Hilbert}

Nesta seção vamos achar uma caracterização de espaços de Hilbert a partir dos espaços reflexivos. Utilizaremos resultados das seções 2.1 e 3.1 e também propriedades da melhor coaproximação vistas nos capítulos anteriores. Em toda a seção trabalharemos $X, V$ e $a$ fixados, onde $X$ é um espaço normado real, $V$ um subespaço de $X$ e $a \in X$.

Teorema 3.2.1. A função $L:] d,+\infty\left[\rightarrow \mathbb{R}\right.$ dada por $L(s)=l_{s}=r_{s}-s$ tem limite finito quando $s \rightarrow \infty$.

Demonstração :

Vamos mostrar que $L$ é crescente. Suponhamos que existam $s$ e $t, s<t$, tais que $l_{s}>l_{t}$. Então é possível escrever

$$
l_{s}-l_{t}=2 \varepsilon \quad \text { com } \varepsilon>0 .
$$

Sejam $c$ um $\varepsilon$-quase centro de $C_{t}$ e $x$ em $C_{s}$ qualquer. Então, como $C_{s} \subset C_{t}$, pela proposição 3.1 .6

$$
\|c-x\| \leq\|a-x\|+r_{t}-t+\varepsilon=\|a-x\|+l_{t}+\varepsilon=\|a-x\|+l_{s}-\varepsilon .
$$

Em particular, como $l_{s}-\varepsilon \leq l_{s}$, pela proposição 3.1.4 deduz-se que $c$ é centro de $C_{s}$.

Como $c$ é centro de $C_{s}$ e portanto $r_{s}=\sup _{x \in C_{s}}\|x-c\|$, podemos tomar uma seqüência $\left\{x_{n}\right\}_{n \in N}$ com $\left\|x_{n}-c\right\| \stackrel{n}{\rightarrow} r_{s}$. Logo, utilizando a parte 1 do corolário 3.1 .10 e a desigualdade (3.7)

$$
r_{s} \leq s+l_{s}-\varepsilon,
$$

e portanto $r_{s} \leq r_{s}-\varepsilon$, o que resulta absurdo. Então concluimos que para $s<t$

$$
l_{s}=L(s) \leq L(t)=l_{t}
$$

Além disso, pela proposição 3.1.7 sabemos que $L$ é limitada superiormente por $d$. Daí,

$$
\lim _{s \rightarrow+\infty} L(s) \in \mathbb{R}
$$


Definição 3.2.2. Chamaremos $l$ a

$$
\lim _{s \rightarrow+\infty} L(s)
$$

O valor $l$, na verdade, deveria ser chamado $l(V, a)$ pois ele depende do subespaço $V$ e do ponto $a$.

Observação 3.2.3. A expressão que aparece na proposição 3.1 .4 pode ser escrita da seguinte maneira:

$$
E_{V}\left(C_{s}\right)=\bigcap_{x \in C_{s}} \bar{B}_{\|a-x\|+l_{s}}(x) \cap V
$$

Portanto. como $l_{s} \leq l$, é fácil ver que

$$
\bigcap_{x \in C_{s}} \bar{B}_{\|a-x\|+l_{s}}(x) \cap V \subset \bigcap_{x \in C_{s}} \bar{B}_{\|a-x\|+l}(x) \cap V .
$$

Definição 3.2.4. Definimos o conjunto $V_{s}$ por

$$
V_{s}=\bigcap_{x \in C_{s}} \bar{B}_{\|a-x\|+l}(x) \cap V .
$$

Definimos o conjunto $V_{\infty}$ por

$$
V_{\infty}=\bigcap_{s>d} V_{s}
$$

Observação 3.2.5. Sejam $s$ e $t$ tais que $d<s<t$. Como $C_{s} \subset C_{t}$ temos que $V_{t} \subset V_{s}$, e se $V_{s} \neq \emptyset$ para todo $s>d$ então $V_{\infty} \neq \emptyset$. É fácil ver que se $v_{0} \in V$

$$
v_{0} \in V_{\infty} \Longleftrightarrow\left\|v_{0}-x\right\| \leq\|x-a\|+l \quad \text { para todo } x \in V .
$$

Teorema 3.2.6. Seja X um espaço de Banach. Então, para qualquer subespaço fechado $V$ de $X$,

$$
a \in D\left(R_{V}\right) \Rightarrow l \leq 0 .
$$

Se $V_{\infty} \neq \emptyset$. vale a implicação contrária e portanto

$$
a \in D\left(R_{V}\right) \Longleftrightarrow l \leq 0 .
$$




\section{Demonstração :}

Se $a \in D\left(R_{V}\right)$, entào existe $v_{0} \in V$ tal que

$$
\left\|x-v_{0}\right\| \leq\|a-x\| \quad \text { para todo } \quad x \in V .
$$

Portanto

$$
r_{s} \leq \sup _{x \in C_{s}}\left\|x-v_{0}\right\| \leq \sup _{x \in C_{s}}\|x-a\| \leq s .
$$

Daí

$$
l_{s}=r_{s}-s \leq 0 .
$$

Logo

$$
l \leq 0 .
$$

Suponhamos agora $l \leq 0$ e $V_{\infty} \neq \emptyset$. Então existe $v_{0} \in V$ tal que satisfaz a inequação que aparece em (3.9). Como $l \leq 0$, para todo $x \in V$ vale

$$
\left\|v_{0}-x\right\| \leq\|a-x\| .
$$

Logo,

$$
a \in D\left(R_{V}\right) .
$$

Definição 3.2.7. Seja $G$ um subespaço de $X$. Uma função $P: X \rightarrow G$ é chamada projeção de $X$ sobre $G$ se satisfaz:

- $P(P(x))=P(x)$ para todo $x \in X$,

- $P$ é linear contínua.

Definição 3.2.8. Um subespaço $G$, é chamado constrito em $X$, se existir uma projeção $P: X \rightarrow G$, com $\|P\|=1$ e $P\left(X^{\prime}\right)=G$. 
Teorema 3.2.9. Se X' é um espaço de Banach que é constrito em $X^{* *}$, então todo subconjunto limitado de $\mathrm{X}$ tem centro de Chebyshev.

\section{Demonstração :}

Para uma demonstraçào ver [Holĩ2] (pag. 184).

Corolário 3.2.10. Seja X um espaço reflexivo. Então todo subconjunto limitado e não vazio $A$ de $X$ tem centro de Chebyshev.

Prova :

Imediata. Como $X$ é reflexivo, consideremos $J^{-1}: X^{* *} \rightarrow X$. Claramente $J^{-1}$ satisfaz as condições do teorema 3.2.9.

Observação 3.2.11. Do corolário anterior se deduz que, se $X$ for reflexivo, $V_{\infty} \neq \emptyset$ para quaisquer $a$ e $V$, pois

$$
\emptyset \neq E_{V}\left(C_{s}\right) \stackrel{(3.8)}{\subset} V_{s}
$$

e daí, pela observação 3.2 .5

$$
V_{\infty} \neq \emptyset
$$

Corolário 3.2.12. Seja $X$ um espaço reflexivo de dimensão maior ou igual a 3. Então as seguintes afirmações são equivalentes:

(i) X é um espaço de Hilbert;

(ii) $l=0$ quaisquer que sejam a $\in V$ :

(iii) $l \leq 0$ quaisquer que sejam a $\in V$.

\section{Prova :}

(i) $\Rightarrow$ (ii)

É imediato pela proposiçào 3.1.11. pois $l_{s}=r_{s}-s=\sqrt{s^{2}-d^{2}}-s \rightarrow 0$ quando $s \rightarrow+\infty$.

(ii) $\Rightarrow$ (iii)

A implicação é óbvia.

(iii) $\Rightarrow($ i $)$

Como $l \leq 0$ para quaisquer $a$ e $V$, pela observação 3.2 .11 e pelo teorema 3.2 .6 temos que $a \in D\left(R_{V}\right)$, ou seja, $D\left(R_{V}\right) \neq \emptyset$. 
Portanto, pelo teorema 2.2.14, X́ é um espaço de Hilbert.

A seguir veremos por que no teorema 3.2 .6 é necessária a hipótese $\operatorname{dim} X \geq 3$.

Teorema 3.2.13. Sejam $X$ um espaço normado, $V$ um subespaço de $X$ de dim=1 e $a \notin V$. Então

(i) existe $c_{0} \in X$ tal que $c_{0}=\lim _{s \rightarrow+\infty} c_{s}$, onde $c_{s}$ é o ponto médio de $C_{s}$;

(ii) $V \perp a-c_{0}$.

\section{Demonstração :}

(i) Seja $c$ tal que $[c]=V$. Portanto, a partir de algum $s_{1}$, existem $\alpha_{s}>0$ e $\beta_{s}<0$ tais que

$$
\left\|\beta_{s} c-a\right\|=\left\|\alpha_{s} c-a\right\|=s .
$$

Como $\alpha_{s} \rightarrow+\infty$ e $\beta_{s} \rightarrow-\infty$ quando $s \rightarrow+\infty$, pela proposição 2.1 .12 sabemos que

$$
\left\|\alpha_{s} c\right\|-\left\|\alpha_{s} c-a\right\|=A\|c\|+\varepsilon_{s}
$$

e

$$
\left\|-\beta_{s} c\right\|-\left\|-\beta_{s} c+a\right\|=-B\|c\|+\eta_{s}
$$

onde $A$ e $B$ são o menor e o maior dos $\alpha^{\prime} s$ que satisfazem $c \perp \alpha c-a, \varepsilon_{s} \rightarrow 0$ e $\eta_{s} \rightarrow 0$ quando $s \rightarrow+\infty$.

Escrito de outra forma

$$
\alpha_{s}\|c\|=A\|c\|+s+\varepsilon_{s}
$$

e

$$
\beta_{s}\|c\|=B\|c\|-s-\eta_{s} .
$$

Daí

$$
\frac{\alpha_{s}+\beta_{s}}{2}\|c\|=\frac{A+B}{2}\|c\|+\gamma_{s}
$$


onde $i s \rightarrow 0$ quando $s \rightarrow+\infty$. Portanto

$$
\begin{gathered}
\lim _{s \rightarrow+\infty} \frac{\alpha_{s}+\beta_{s}}{2}=\frac{A+B}{2} \text { e como } c_{s}=\frac{\alpha_{s}+\beta_{s}}{2} c, \\
\lim _{s \rightarrow+\infty} c_{s}=\frac{A+B}{2} c=c_{0} .
\end{gathered}
$$

(ii) Além disso, pelo lema 2.1.9 temos que $c \perp c_{0}-a$ pois $A \leq \frac{A+B}{2} \leq B$, donde se deduz

$$
V \perp c_{0}-a \text {. }
$$

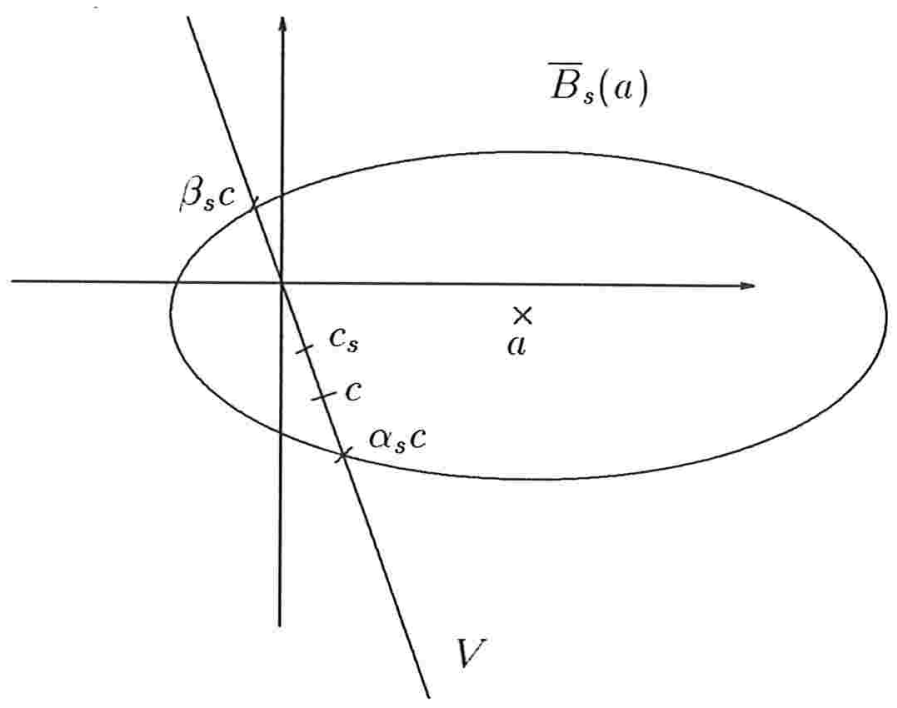

\section{Figura 3.6.}

Observação 3.2.14. Se no teorema 3.2 .13 considerarmos $V_{1}=[\{a\} \cup V]$, teríamos que $V_{1}$ é um espaço reflexivo com $l \leq 0$ para quaisquer $a$ e $V$ (ver teorema 3.2.6). Mas $V_{1}$ não precisa ser um espaço de Hilbert, o que mostra a necessidade da hipótese $\operatorname{dim} X \geq 3$ no corolário 3.2.12. 


\section{Capítulo 4}

\section{Caracterizações de Melhor Coaproximação e Aplicações}

Neste capítulo estudaremos condições necessárias e suficientes para a existência de melhor coaproximação utilizando condições já conhecidas para a existência da melhor aproximação.

Primeiramente, provaremos algumas proposições que vinculam a melhor coaproximação de um ponto em um subespaço com a melhor aproximação desse mesmo ponto em um outro subespaço que será definido oportunamente. Para isso, utilizaremos resultados conhecidos sobre a melhor aproximação, cujas provas omitiremos por serem bastante longas. Citaremos, em cada caso, as páginas onde elas podem ser encontradas em [Sin70].

Além disso, aplicando esses resultados, estudaremos a existência e caracterizações de coaproximações polinômicas no espaço $\left(C[0,1]\right.$, \|\|$\left._{\infty}\right)$ e uma caracterização de espaços estritamente convexos.

Em todo o capítulo utilizaremos as conhecidas notações $B_{X}$ e $S_{X}$ :

$$
B_{X}=\{x \in X:\|x\| \leq 1\} \text { e } S_{X}=\{x \in X:\|x\|=1\} .
$$




\subsection{Caracterizações de Melhor Coaproximação}

Nesta seção estudaremos caracterizaçòes de melhor coaproximação utilizando uma caracterização de melhor aproximação. Os resultados serão muito importantes nas sações 4.2 e 4.3 .

Proposição 4.1.1. Sejam $X$ um espaço normado. G um subespaço de $X$ e $x \in X \backslash G$. Entiòo

$$
R_{G}(x)=\left\{g_{0} \in G: g_{0} \in \bigcap_{g \in G} P_{\mathfrak{l}} \cdot(g)\right\}
$$

onde $V=\left\{g_{0}+\alpha\left(x-g_{0}\right): \alpha \in \mathbb{R}\right\}$.

\section{Prova :}

Seja $g_{0} \in R_{G}(x)$. Provaremos que $g_{0} \in P_{V}(g)$ para qualquer $g \in G$.

Para quaisquer $g \in G$ e $\alpha \in \mathbb{R}$ temos

$\left\|g-g_{0}-\alpha\left(x-g_{0}\right)\right\| \geq\left\|g-g_{0}\right\|$, pois $G \perp x-g_{0}$, e portanto

$$
g_{0} \in \bigcap_{g \in G} P_{V}(g) .
$$

Reciprocamente, se $g_{0} \in G$ e satisfaz a expressão (4.2). temos que para todo $g \in G$ e todo $\alpha \in \mathbb{R}$,

$$
\left\|g-\alpha x-(1-\alpha) g_{0}\right\| \geq\left\|g-g_{0}\right\| .
$$

Daí, tomando $\alpha=1$, concluimos que para todo $g \in G$

$$
\|g-x\| \geq\left\|g-g_{0}\right\|,
$$

ou seja,

$$
g_{0} \in R_{G}(x)
$$




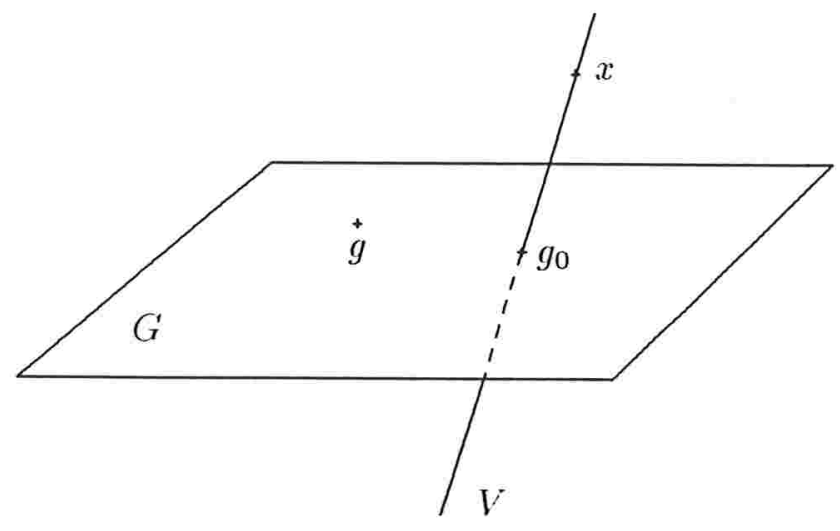

\section{Figura 4.1.}

Corolário 4.1.2. Sejam $X$ um espaço normado, $G$ um subespaço de $X, x \in X \backslash G$ e $g_{0} \in G$.

Então

$$
g_{0} \in R_{G}(x) \Longleftrightarrow G \subset P_{\left[x-g_{0}\right]}^{-1}(0)=\left\{z \in X: 0 \in P_{\left[x-g_{0}\right]}(z)\right\}
$$

onde $\left[x-g_{0}\right]=\left\{\beta\left(x-g_{0}\right): \beta \in \mathbb{R}\right\}$.

\section{Prova :}

Sendo $V$ como na proposição 4.1.1, basta observar que $g_{0} \in P_{V}(g)$ se e somente se $0 \in P_{\left[x-g_{0}\right]}\left(g-g_{0}\right)$ e utilizar a proposição 4.1.1.

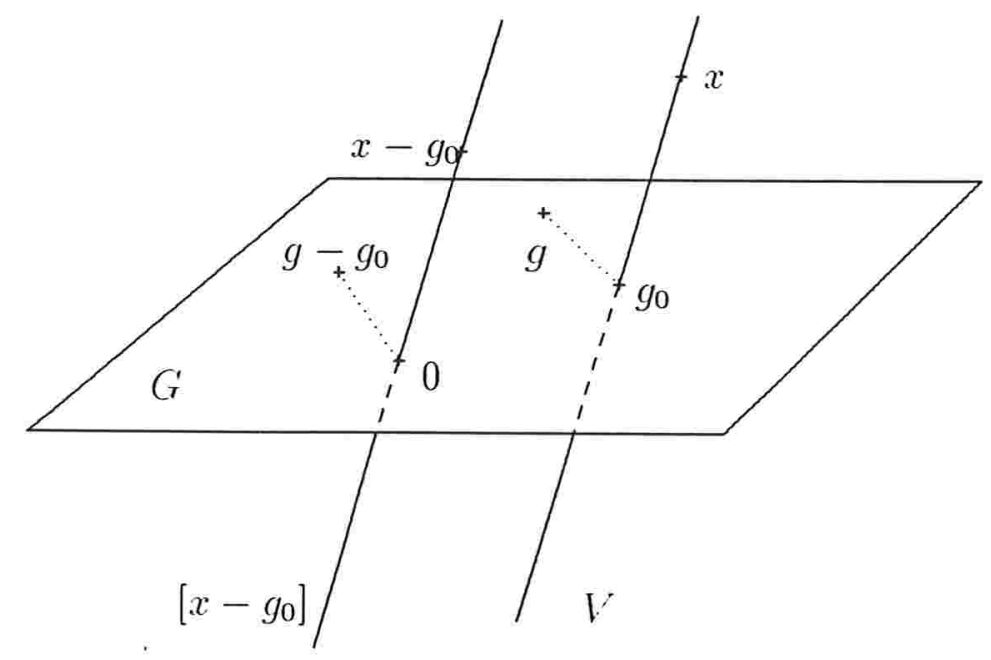

Figura 4.2. 
Teorema 4.1.3. Sejam $X$ um espaço normado de dimensão maior ou igual que 2 , $V$ um subespaço afim de $X$ de dimensão $1, z \in X$ e $v_{0} \in V$. Então as seguintes afirmações são equivalentes:

(i) $v_{0} \in P_{V}(z)$;

(ii) existe $f \in X^{*}$ tal que:

1) $\|f\|=1$,

2) $f\left(v-v_{0}\right)=0$, para todo $v \in V$,

3) $f\left(z-v_{0}\right)=\left\|z-v_{0}\right\|$.

\section{Demonstração :}

Consideraremos dois casos: a) $z \in X \backslash V$ e b) $z \in V$.

Para o caso a) $z \in X \backslash V$, ver [Sin70] (teorema 5.1, pag.138).

Agora provaremos o caso $b) z \in V$.

(i) $(\Rightarrow)$ (ii)

Temos que $z \in V$ e $v_{0} \in P_{V}(z)$, logo $v_{0}=z$. Seja $G$ um subespaço de dimensão 2 de $X$ tal que $V \subset G$. Como $V$ é um subespaço afim de $G$ de dimensão 1 , é em particular um hiperplano afim de $G$. Portanto existem $\widehat{f} \in G^{*}$ não nulo e $c \in \mathbb{R}$ tais, que $V=\widehat{f}^{-1}(c)$. Seja $\tilde{f}=\frac{\hat{f}}{\|\hat{f}\|}$. Agora, pelo teorema de Hann-Banach, estendemos $\tilde{f}$ a todo $X$ e seja $f$ tal extensão. É fácil ver que $f$ satisfaz as propriedades 1), 2) e 3).

(ii) $(\Rightarrow)$ (i)

Agora temos que $z \in V$ e que existe $f \in X^{*}$ tal que satisfaz as propriedades 1 ), 2) e 3).

Por 2), como $z \in V$, temos que

$$
f\left(z-v_{0}\right)=0
$$

Logo, por 3),

$$
\left\|z-v_{0}\right\|=0
$$

Portanto $z=v_{0}$ e daí

$$
v_{0} \in P_{V}(z)
$$

Teorema 4.1.4. Sejam X um espaço normado, $G$ um subespaço de $X$ e $x \in X \backslash G$. Então, se $g_{0} \in G$, as seguintes afirmações são equivalentes: 
(i) $g_{0} \in R_{G}(x)$.

(ii) Para cada $g \in G$ existe um funcional linear $f^{g} \in X^{*}$ tal que

$$
\begin{gathered}
\left\|f^{g}\right\|=1, \\
f^{g}(x)=f^{g}\left(g_{0}\right), \\
f^{g}(g)=\|g\| .
\end{gathered}
$$

(iii) Para cada $g \in G$ existe um funcional linear $f^{g} \in X^{*}$ tal que

$$
\begin{gathered}
\left\|f^{g}\right\|=1, \\
f^{g}(x)=f^{g}\left(g_{0}\right), \\
\left|f^{g}(g)\right|=\|g\| .
\end{gathered}
$$

Demonstração :

(i) $\Longleftrightarrow$ (ii)

Seja $g \in G$ qualquer.

Sabemos pela proposiçào 4.1.1 que, sendo $V=\left\{g_{0}+\alpha\left(x-g_{0}\right): \alpha \in \mathbb{R}\right\}$,

$$
g_{0} \in R_{G}(x) \text { se e somente } g_{0} \in P_{V}\left(g_{1}\right) \text { para todo } g_{1} \in G,
$$

o que, pelo teorema 4.1.3, é equivalente com:

- para todo $g_{1} \in G$, existe $f \in X^{*}$ tal que

1) $\|f\|=1$,

2) $f\left(v-g_{0}\right)=0$ para todo $v$ da forma $g_{0}+\alpha\left(x-g_{0}\right)$,

3) $f\left(g_{1}-g_{0}\right)=\left\|g_{1}-g_{0}\right\|$.

Observando que por hipótese $g_{0} \in G$, para todo $g \in G$ podemos escrever $g+g_{0}=g_{1}$, e obtemos também a equivalência com:

- para todo $g \in G$, existe $f^{g} \in X^{*}$ tal que 
1') $\left\|f^{g}\right\|=1$,

2') $f^{g}\left(\alpha x+(1-\alpha) g_{0}-g_{0}\right)=\alpha f^{g}\left(x-g_{0}\right)=0$ para todo $\alpha \in \mathbb{R}$,

3') $f^{g}(g)=\|g\|$.

Logo, (i) é equivalente a (ii).

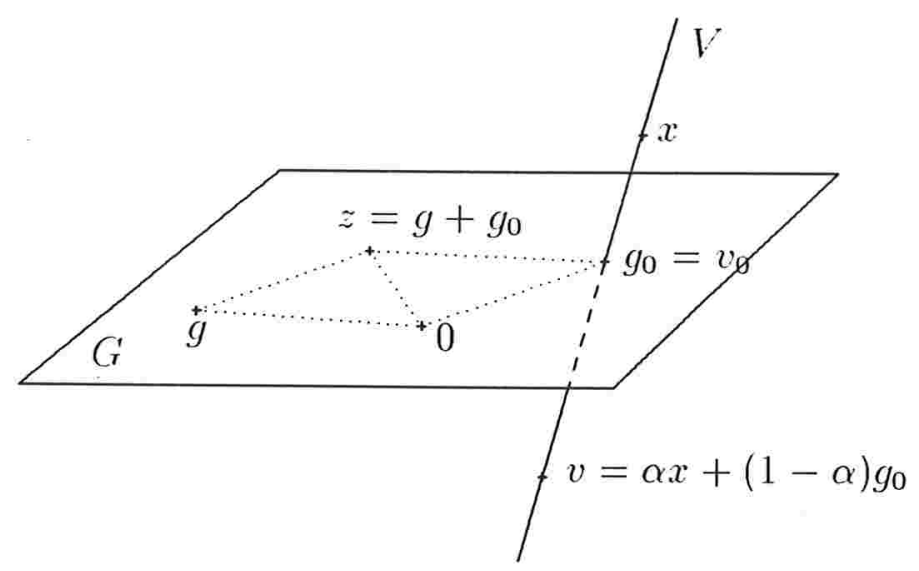

Figura 4.3.

(ii) $\Longleftrightarrow$ (iii)

Imediata. No pior dos casos devemos trocar o sinal da $f$.

Lema 4.1.5. Sejam $X$ um espaço de Banach de dimensão 2 e $\varphi \in X^{*}$ tal que $\|\varphi\|=$ 1. Então existem 2 pontos extremais $\varphi_{1}, \varphi_{2} \in S_{X^{*}}$ (não necessariamente distintos) $e$ $\lambda_{1}$ e $\lambda_{2} \in \mathbb{R}^{+} \operatorname{com} \lambda_{1}+\lambda_{2}=1$, tais que

$$
\varphi=\lambda_{1} \varphi_{1}+\lambda_{2} \varphi_{2}
$$

Prova :

Para uma demonstração deste lema ver [Sinio] (lema 1.1, pag.166).

Lema 4.1.6. Sejam X um espaço normado, $G$ um subespaço de $X$ e $\varphi$ um ponto extremal de $S_{G^{*}}$. Então existe uma extensão de $\varphi$ para um ponto extremal $\hat{\varphi}$ de $S_{X^{*}}$. 


\section{Prova :}

Para uma demonstração deste lema ver [Sinĩ0] (lema 1.2, pag.16i).

Teorema 4.1.7. Sejam X um espaço de Banach de dimensão maior ou igual a 2, $V$ um subespaço afim de $X$ de dimensão 1, $y \in X$ e $v_{0} \in V$. Então as seguintes afirmações são equivalentes:

(i) $v_{0} \in P_{V}(y)$;

(ii) existem $f_{1}$ e $f_{2}$ pontos extremais de $S_{X} \cdot$ e $\lambda_{1}, \lambda_{2} \in \mathbb{R}^{+}$tais que:

1) $\lambda_{1}+\lambda_{2}=1$

2) $\left(\lambda_{1} f_{1}+\lambda_{2} f_{2}\right)\left(v-v_{0}\right)=0$, para todo $v \in V$,

3) $\left(\lambda_{1} f_{1}+\lambda_{2} f_{2}\right)\left(y-v_{0}\right)=\left\|y-v_{0}\right\|$.

\section{Demonstração :}

Consideraremos dois casos: a) $y \in X \backslash V$ e b) $y \in V$.

Para o caso a) $z \in X \backslash V$, ver [Sin70] (teorema 4.1, pag.242). Agora provaremos o caso b) $z \in V$.

(i) $(\Rightarrow)$ (ii)

Seja $G$ subespaço de dimensão 2 de $X$ tal que $V \subset G$.

Como $v_{0} \in P_{V}(y)$, pelo teorema 4.1.3 sabemos que existe $f \in G^{*}$ tal que

$$
\begin{gathered}
\|f\|=1, \\
f\left(v-v_{0}\right)=0 \text { para todo } v \in V, \\
f\left(x-v_{0}\right)=\left\|x-v_{0}\right\| .
\end{gathered}
$$

Portanto, pelo lema 4.1.5 existem $\varphi_{1}, \varphi_{2}$ pontos extremais de $S_{G^{*}}$ e $\lambda_{1}, \lambda_{2} \in \mathbb{R}^{+}$com $\lambda_{1}+\lambda_{2}=1$ tais que

$$
f=\lambda_{1} \varphi_{1}+\lambda_{2} \varphi_{2}
$$

Agora, utilizando o lema 4.1.6 estendemos $\varphi_{1}$ e $\varphi_{2}$ para $f_{1}$ e $f_{2}$, pontos extremais de $S_{X^{*}}$. E fácil ver que

1) $\lambda_{1}+\lambda_{2}=1$

2) $\left(\lambda_{1} f_{1}+\lambda_{2} f_{2}\right)\left(v-v_{0}\right)=\left(\lambda_{1} \varphi_{1}+\lambda_{2} \varphi_{2}\right)\left(v-v_{0}\right)=0$, para todo $v \in V$, 
3) $\left(\lambda_{1} f_{1}+\lambda_{2} f_{2}\right)\left(y-v_{0}\right)=\left\|y-v_{0}\right\|=0$.

(ii) $(\Rightarrow)$ (i)

Agora temos que $y \in V$ e que existe $f \in X^{*}$ tal que satisfaz as propriedades 1 ), 2) e 3).

Por 2), como $y \in V$, temos que

$$
f\left(y-v_{0}\right)=0
$$

Logo, por 3)

$$
\left\|y-v_{0}\right\|=0
$$

Portanto $x=v_{0}$ e daí

$$
\iota_{0} \in P_{V}(y) .
$$

Teorema 4.1.8. Sejam X um espaço de Banach de dimensão maior ou igual que 2, $G$ um subespaço de $X$ e $x \in X \backslash G$. Um elemento go de $G$ é tal que $g_{0} \in$ $R_{G}(x)$ se e somente se para cada $g \in G$, existem dois pontos extremais $f_{1}^{g}$ e $f_{2}^{g}$ (não necessariamente distintos) de $S_{X}$ e dois números $\lambda_{1}^{g}$ e $\lambda_{2}^{g}$ em $\mathbb{R}^{+}$, com $\lambda_{1}^{g}+\lambda_{2}^{g}=1$, tais que

$$
\begin{gathered}
\lambda_{1}^{g} f_{1}^{g}(x)+\lambda_{2}^{g} f_{2}^{g}(x)=\lambda_{1}^{g} f_{1}^{g}\left(g_{0}\right)+\lambda_{2}^{g} f_{2}^{g}\left(g_{0}\right), \\
\lambda_{1}^{g} f_{1}^{g}(g)+\lambda_{2}^{g} f_{2}^{g}(g)=\|g\| .
\end{gathered}
$$

Demonstração :

Sabemos pela proposição 4.1 .1 que, sendo $V=\left\{g_{0}+\alpha\left(x-g_{0}\right): \alpha \in \mathbb{R}\right\}$,

$$
g_{0} \in R_{G}(x) \text { se e somente } g_{0} \in P_{V}\left(g_{1}\right) \text { para todo } g_{1} \in G \text {, }
$$

o que, pelo teorema 4.1.7, é equivalente com:

- para todo $g_{1} \in G$, existem $f_{1} . f_{2}$ pontos extremais de $S_{X^{*}}$ e $\lambda_{1}, \lambda_{2} \in \mathbb{R}$ tais que

1) $\lambda_{1}+\lambda_{2}=1$,

2) $\left(\lambda_{1} f_{1}+\lambda_{2} f_{2}\right)\left(v-g_{0}\right)=0$, para todo $v$ da forma $\left.\left.g_{0}+\alpha\right) x-g_{0}\right)$, , 
3) $\left(\lambda_{1} f_{1}+\lambda_{2} f_{2}\right)\left(g_{1}-g_{0}\right)=\left\|g_{1}-g_{0}\right\|$.

Observando que por hipótese $g_{0} \in G$, para qualquer $g \in G$ podemos escrever $g+g_{0}=g_{1}$, e obtemos também a equivalência com: que

- para todo $g \in G$, existem $f_{1}^{g}, f_{2}^{g}$ pontos extremais de $S_{X^{*}}$ e $\lambda_{1}^{g}, \lambda_{2}^{g} \in \mathbb{R}$ tais

1') $\lambda_{1}^{g}+\lambda_{2}^{g}=1$,

Q') $\left(\lambda_{1} f_{1}^{g}+\lambda_{2} f_{2}^{g}\right)\left(\alpha x+(1-\alpha) g_{0}-g_{0}\right)=\alpha\left(\lambda_{1} f_{1}^{g}+\lambda_{2} f_{2}^{g}\right)\left(x-g_{0}\right)=0$, para todo $\alpha \in \mathbb{R}$,

3') $\left(\lambda_{1} f_{1}^{g}+\lambda_{2} f_{2}^{g}\right)(g)=\|g\|$. 


\subsection{Melhor Coaproximação Polinômica}

Nesta seção, utilizando os teoremas 4.1.4 e 4.1.8 estudaremos condições de existência e caracterizações da melhor coaproximação em $X=\left(C[0,1],\|\|_{\infty}\right)$ no subespaço dos polinômios $\mathcal{P}$ e em alguns subespaços de $\mathcal{P}$.

Teorema 4.2.1. Sejam. $X=\left(C[a, b],\|\|_{\infty}\right), G$ um subespaço de $X$ e $x \in X \backslash G$. Um elemento $g_{0}$ de $G$ satisfaz que $g_{0} \in R_{G}(x)$ se e somente se para cada $g \in G$, existem dois pontos $q_{1}^{g}$ e $q_{2}^{g}$ de $[a, b]$ e dois números $\mu_{1}^{g}$ e $\mu_{2}^{g}$ em $\mathbb{R}$. com $\left|\mu_{1}^{g}\right|+\left|\mu_{2}^{g}\right|=1$, tais que

$$
\begin{gathered}
\mu_{1}^{g} x\left(q_{1}^{g}\right)+\mu_{2}^{g} x\left(q_{2}^{g}\right)=\mu_{1}^{g} g_{0}\left(q_{1}^{g}\right)+\mu_{2}^{g} g_{0}\left(q_{2}^{g}\right), \\
\mu_{1}^{g} g\left(q_{1}^{g}\right)+\mu_{2}^{g} g\left(q_{2}^{g}\right)=\|g\|_{\infty} .
\end{gathered}
$$

\section{Demonstração :}

Sabemos por [D-S58] (pag.441) que um funcional linear $f \in X^{* *}$ é um ponto extremal de $B_{X}$. se e somente se existe um $q \in[a, b]$ e um numero real $\varepsilon$ com $|\varepsilon|=1$, tal que para todo $y \in X$

$$
f(y)=\varepsilon y(q)
$$

\section{$(\Rightarrow)$}

Se $g_{0} \in R_{G}(x)$, para cada $g \in G$ existem $f_{i}^{g}$ e $\lambda_{i}^{g}$ como no teorema 4.1.8, $i=1,2$. Escrevendo os extremais $f_{i}^{g}$ por $f_{i}^{g}(y)=\varepsilon_{i}^{g} y\left(q_{i}^{g}\right)$, definindo $\mu_{i}^{g}=\lambda_{i}^{g} \varepsilon_{i}^{g}, i=1,2$, e substituindo em (4.4) e (4.5), obtemos imediatamente as expressões (4.6) e (4.7).

\section{$(\Leftarrow)$}

Reciprocamente, se $q_{i}^{g}$ e $\mu_{i}^{g}$ estão nas condições de (4.6) e (4.7), então, para cada $g \in G$, definindo

$$
f_{i}^{g}(y)= \begin{cases}\left(\operatorname{sg} \mu_{i}^{g}\right) y\left(q_{i}^{g}\right), & \text { se } \mu_{i}^{g} 1 \neq 0 \\ y\left(q_{i}^{g}\right) & \text { se } \mu_{i}^{g}=0,\end{cases}
$$

e $\lambda_{i}^{g}=\left|\mu_{i}^{g}\right|, i=1,2$ nos dois casos, obtemos $\lambda_{1}^{g}+\lambda_{2}^{g}=1, f_{i}^{g}$ extremais de $S_{X^{*}}$ e as expressões (4.4) e (4.5). Logo, pelo teorema 4.1.8, concluimos que $g_{0} \in R_{G}(x)$. 
Observação 4.2.2. Para quaisquer $g \in G, q_{1}^{g}, q_{2}^{g} \in[a, b]$ e $\mu_{1}^{g}, \mu_{2}^{g}$ como no teorema 4.2 .1 , se $\mu_{1}^{g} \neq 0$, então $\left|g\left(q_{1}^{g}\right)\right|=\|g\|_{\infty}$. De fato, $\left|g\left(q_{1}^{g}\right)\right| \leq\|g\|_{\infty}$ e supondo que $\left|g\left(q_{1}^{g}\right)\right|<\|g\|_{\infty}$, obtemos

$$
\begin{gathered}
\left|\mu_{1}^{g} g\left(q_{1}^{g}\right)+\mu_{2}^{g} g\left(q_{2}^{g}\right)\right| \leq\left|\mu_{1}^{g} g\left(q_{1}^{g}\right)\right|+\left|\mu_{2}^{g} g\left(q_{2}^{g}\right)\right|< \\
<\left|\mu_{1}^{g}\right|\|g\|+\left|\mu_{2}^{g}\right|\left|g\left(q_{2}^{g}\right)\right| \leq\left(\left|\mu_{1}^{g}\right|+\left|\mu_{2}^{g}\right|\right)\|g\|_{\infty}=\|g\|_{\infty},
\end{gathered}
$$

o que contradiz a expressão (4.7). Analogamente, se $\mu_{2}^{g} \neq 0,\left|g\left(q_{2}^{g}\right)\right|=\|g\|_{\infty}$.

Observação 4.2.3. Se $\left|g\left(q_{1}^{g}\right)\right|=\|g\|_{\infty}$, então claramente a expressão (4.7) está satisfeita com $\mu_{1}^{g}=\operatorname{sg} g\left(q_{1}^{g}\right)$ e $\mu_{2}^{g}=0$. Análogo para $i=2$.

A seguir veremos que, apesar da observação 4.2.3, existem casos em que devem ser dois os números $q_{i}^{g}$ mencionados nas hipóteses do teorema 4.2.1.

Exemplo 4.2.4. Sejam $X=\left(C[0,1],\|\|_{\infty}\right), y(t)=2 t-1, G=[y]$ e $x(t)=\left|t-\frac{1}{2}\right|$. Primeiro vejamos que $G \perp x$. Pela homogeneidade da ortogonalidade basta verificar que $y \perp x$. Com efeito, se $\alpha \geq 0$, então

$$
\|y+\alpha x\|_{\infty} \geq(y+\alpha x)(1) \geq y(1)=1=\|y\|_{\infty},
$$

e se $\alpha \leq 0$,

$$
\|y+\alpha x\|_{\infty} \geq(y+\alpha x)(0) \geq y(0)=1=\|y\|_{\infty},
$$

o que prova nossa afirmação.

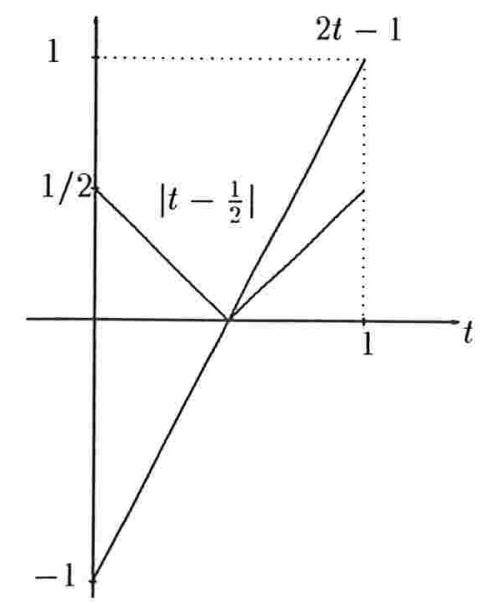

Figura 4.4 . 
Como $G \perp x$, então $g_{0}=0 \in R_{G}(x)$. Agora observemos que se as expressões (4.6) $\mathrm{e}$ (4.7) estivessem satisfeitas com $\left|\mu_{1}^{g}\right|=1$ e $\mu_{2}^{g}=0$, então por (4.i), $q_{1}^{g}$ deveria ser 0 ou 1 , que são os pontos onde $\|g\|_{\infty}$ é atingida, para qualquer $g \in G$. Mas esses valores não satisfariam (4.6) pois $g_{0}(0)=0 \neq \frac{1}{2}=x(0)$ e $g_{0}(1)=0 \neq \frac{1}{2}=x(1)$. De fato, os valores que satisfazem as equações (4.6) e (4.7), são $q_{1}^{g}=0, q_{2}^{g}=1, \mu_{1}^{g}=-\frac{1}{2} \mathrm{e}$ $\mu_{2}^{g}=\frac{1}{2}$, se $g(t)=k(2 t-1)$ para $k \geq 0$ e $q_{1}^{g}=0, q_{2}^{g}=1, \mu_{1}^{g}=\frac{1}{2}$ e $\mu_{2}^{g}=-\frac{1}{2}$, para $k<0$.

Teorema 4.2.5. Sejam $X=\left(C[a, b],\|\|_{\infty}\right), G$ um subespaço de $X$ e $x \in X \backslash G$, que tem (pelo menos uma) melhor coaproximaçào $g_{0} \mathrm{em} G$. Se existe $h \in G$ tal que $|h|$ atinge seu máximo absoluto estrito em algum $t_{0} \in[a, b]$ (i.e. $\left|h\left(t_{0}\right)\right|=\|h\|>|h(q)|$ para todo $q \neq t_{0}, q \in[a, b]$ ), então

$$
g_{0}\left(t_{0}\right)=x\left(t_{0}\right)
$$

\section{Demonstração :}

Sejam $q_{i}^{h}$ e $\mu_{i}^{h}(i=1,2)$ nas condições do teorema 4.2.1. Então, pela observação 4.2.2, para cada $i=1,2$ tal que $\mu_{i}^{h} \neq 0$ temos que $\left|h\left(q_{i}^{h}\right)\right|=\|h\|_{\infty}$. Logo, se $\mu_{i}^{h} \neq 0$, $q_{i}^{h}=t_{0}$.

Usando agora a expressão (4.7), temos, quaisquer que sejam os $\mu_{i}^{h}$,

$$
\|h\|_{\infty}=\mu_{1}^{h} h\left(q_{1}^{h}\right)+\mu_{2}^{h} h\left(q_{2}^{h}\right)=\mu_{1}^{h} h\left(t_{0}\right)+\mu_{2}^{h} h\left(t_{0}\right),
$$

e portanto, como $\|h\|_{\infty} \neq 0$, temos que $\mu_{1}^{h}+\mu_{2}^{h} \neq 0$.

Mas, pela equação (4.6), $\mu_{1}^{h} x\left(t_{0}\right)+\mu_{2}^{h} x\left(t_{0}\right)=\mu_{1}^{h} g_{0}\left(t_{0}\right)+\mu_{2}^{h} g_{0}\left(t_{0}\right)$, e então

$$
x\left(t_{0}\right)=g_{0}\left(t_{0}\right) .
$$

Corolário 4.2.6. Sejam $X=\left(C[a, b],\|\|_{\infty}\right)$ e $G$ um subespaço de $X$ tal que para todo ponto $t$ de um conjunto $M$ denso em $[a, b]$ existe uma função $f_{t} \in G$ tal que $\left|f_{t}\right|$ atinge seu máximo absoluto estrito em $t$. Então, para todo $x \in X \backslash G$,

$$
R_{G}(x)=\emptyset \text {. }
$$

\section{Prova :}

Seja $x \in X \backslash G$ e suponhamos que existe $g_{0} \in R_{G}(x)$. Entào, pelo corolório 4.2.5, temos que $x(t)=g_{0}(t)$ para todo $t \in M$. Logo, pela continuidade de $x$, concluimos que $x=g_{0} \in G$, o que resulta absurdo. Portanto $R_{G}(x)=\emptyset$. 
Observação 4.2.7. Lembremos do exemplo 2.4. Nele $G=\{f \in X: f(0)=0\}$ e havíamos visto que $R_{G}(a)=\emptyset$, onde $a \equiv 1$. É fácil ver que para todo racional $t \mathrm{em}$ $(0,1]$, existe uma função $y_{t} \in G$ que atinge o máximo absoluto em $t$. Pelo corolário anterior, $R_{G}(x)=\emptyset$ para todo $x \in X \backslash G$ e nào só para $a$. (figura 4.5)

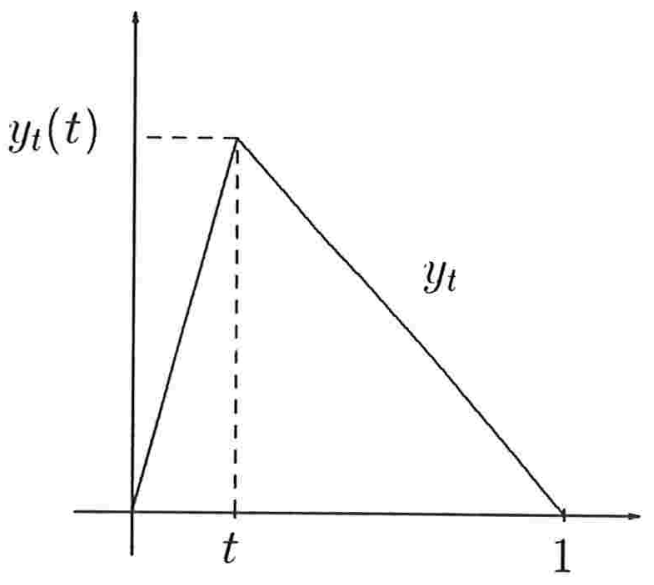

Figura 4.5.

Corolário 4.2.8. Seja $X=\left(C[a, b],\|\|_{\infty}\right)$ e seja $G$ um subespaço de $X$ contendo as funções $g_{1}(t)=1, g_{2}(t)=t$ e $g_{3}(t)=t^{2}$. Então para toda $x \in X \backslash G$

$$
R_{G}(x)=\emptyset .
$$

\section{Prova :}

É fácil ver que para todo $s \in[0,1]$ existe uma função $g_{s}$ da forma $g_{s}(t)=a t^{2}+b t+c$ que atinge um máximo absoluto estrito em $s$. Logo, pelo corolário 4.2.6, temos que para toda $x \in X \backslash G$

$$
R_{G}(x)=\emptyset \text {. }
$$

Proposição 4.2.9. Sejam $X=\left(C[a, b],\|\|_{\infty}\right), G=[1]$ e $x \in X \backslash G$. Então

$$
R_{G}(x)=\left\{g_{0} \in G: g_{0} \equiv c, \text { com } \min _{t \in[a, b]} x(t) \leq c \leq \max _{t \in[a, b]} x(t)\right\} .
$$

\section{Prova :}

Sabemos pelo teorema 2.2 .5 que $R_{G}(x) \neq \emptyset$. Seja então $g_{0} \in R_{G}(x), g_{0}(t)=c$ para todo $t \in[0,1]$. Para $g(t)=1 \in G$, tomemos os $q_{i}^{g}$ e os $\mu_{i}^{g}$ como no teorema 4.2.1. 
Então, pela equação (4.7), temos que $\mu_{1}^{g}+\mu_{2}^{g}=1$, e como além disso $\left|\mu_{1}^{g}\right|+\left|\mu_{2}^{g}\right|=1$, concluimos que $\mu_{i}^{g} \geq 0$. Em conseqüência

$$
\min _{t \in[a, b]} x(t) \leq \mu_{1}^{g} x\left(q_{1}^{g}\right)+\mu_{2}^{g} x\left(q_{2}^{g}\right) \leq \max _{t \in[a . b]} x(t)
$$

$\mathrm{e}$

$$
\mu_{1}^{g} g_{0}\left(q_{1}^{g}\right)+\mu_{2}^{g} g_{0}\left(q_{2}^{g}\right)=\left(\mu_{1}^{g}+\mu_{2}^{g}\right) c=c .
$$

Daí, utilizando a equação (4.6), obtemos

$$
\min _{t \in[a, b]} x(t) \leq c \leq \max _{t \in[a, b]} x(t)
$$

Reciprocamente, tomemos $g_{0} \in G, g_{0} \equiv c \in G$, com $c$ satisfazendo

$$
\min _{t \in[a, b]} x(t) \leq c \leq \max _{t \in[a, b]} x(t)
$$

Então, como $x$ é contínua, existe $q_{0} \in[a, b]$ tal que $x\left(q_{0}\right)=c$. Portanto, para qualquer $g \in G$, tomamos $q_{1}^{g}=q_{2}^{g}=q_{0}, \mu_{1}^{g}=1$ e $\mu_{2}^{g}=0$. É fácil ver que com esses valores as equações (4.6) e (4.7) estão satisfeitas. Logo, pelo teorema 4.2.1, deduz-se que

$$
g_{0} \in R_{G}(x) .
$$

Observação 4.2.10. Lembremos do exemplo 1.2.7. Lá, $G=[1]$. Não é só para a função $f(t)=e^{t}$ que temos $c \in R_{G}(f)$, para todo $c \in f([0,1])$, pois, segundo a proposição 4.2.9, isso acontece para qualquer elemento de $X \backslash G$.

Proposição 4.2.11. Sejam $X=\left(C[0,1],\|\|_{\infty}\right), n \geq 1, G=\left[t^{n}\right]$ e $x \in X \backslash G$. Então $R_{G}(x)$ é unitário, dado por

$$
R_{G}(x)=\left\{x(1) t^{n}\right\}
$$

\section{Prova :}

De novo, pelo teorema 2.2.5, sabemos que $R_{G}(x) \neq \emptyset$. Seja então $g_{0} \in R_{G}(x)$, que é da forma $g_{0}(t)=\alpha_{0} t^{n}$. Então, como $g(t)=t^{n}$ atinge seu máximo absoluto em $t=1$, pelo corolário 4.8 segue que $x(1)=g_{0}(1)=\alpha_{0}$. Logo $g_{0}(t)=x(1) t^{n}$, ou seja, $R_{G}(x)=\left\{x(1) t^{n}\right\}$. 
Proposição 4.2.12. Sejam $X=\left(C[a, b],\|\|_{\infty}\right), G=[1, z]$, onde

$$
\min _{t \in[a, b]} z(t)=z\left(t_{1}\right)<z\left(t_{2}\right)=\max _{t \in[a, b]} z(t)
$$

e seja $x \in X \backslash G$. Entĩo $g_{0} \in R_{G}(x)$, onde

$$
g_{0}(t)=\frac{x\left(t_{1}\right) z\left(t_{2}\right)-x\left(t_{2}\right) z\left(t_{1}\right)+\left[x\left(t_{2}\right)-x\left(t_{1}\right)\right] z(t)}{z\left(t_{2}\right)-z\left(t_{1}\right)}, t \in[0,1] .
$$

Além disso, se existem $g_{1}, g_{2} \in G$ tais que $\left|g_{i}\right|$ atingem seus máximos absolutos estritos em $t_{i}, i=1,2$, então $R_{G}(x)$ é um conjunto unitário para cada $x \in X \backslash G$, ou seja, $R_{G}(x)=\left\{g_{0}\right\}$.

\section{Prova :}

Seja $g_{0}$ definida como em (4.10). Então $g_{0} \in G, g_{0}\left(t_{1}\right)=x\left(t_{1}\right)$ e $g_{0}\left(t_{2}\right)=x\left(t_{2}\right)$. Também, para toda $g=\alpha_{1}+\alpha_{2} z \in G$, temos por (4.9) que ou $\left|g\left(t_{1}\right)\right|=\|g\|_{\infty}$ ou $\left|g\left(t_{2}\right)\right|=\|g\|_{\infty}$. No primeiro caso, tomamos $q_{1}^{g}=t_{1}, q_{2}^{g}=t_{2}, \mu_{1}^{g}=\operatorname{sg} g\left(t_{1}\right)$ e $\mu_{2}^{g}=0$. No segundo, tomamos os mesmos $q_{i}^{g}, \mu_{1}^{g}=0$ e $\mu_{2}^{g}=\operatorname{sg} g\left(t_{2}\right)$. Desta maneira estão satisfeitas as equações (4.6) e (4.7). Portanto, pelo teorema 4.2.1, temos que

$$
g_{0} \in R_{G}(x) .
$$

Suponhamos agora que existam $g_{1}, g_{2} \in G$ tais que $\left|g_{i}\right|$ atingem seus máximos absolutos estritos em $t_{i} \quad i=1,2$. Então, pelo teorema 4.2 .5 , temos que toda $g_{0} \in$ $R_{G}(x)$ satisfaz

$$
x\left(t_{i}\right)=g_{0}\left(t_{i}\right), i=1,2 .
$$

Sejam $g_{0}$ e $h_{0} \in R_{G}(x)$. Eles podem ser expressos assim:

$$
g_{0}=\alpha_{1}+\alpha_{2} z \quad \text { e } h_{0}=\alpha_{1}^{\prime}+\alpha_{2}^{\prime} z .
$$

Então

$$
\alpha_{1}+\alpha_{2} z\left(t_{1}\right)=x\left(t_{1}\right)=\alpha_{1}^{\prime}+\alpha_{2}^{\prime} z\left(t_{1}\right)
$$

$\mathrm{e}$

$$
\alpha_{1}+\alpha_{2} z\left(t_{2}\right)=x\left(t_{2}\right)=\alpha_{1}^{\prime}+\alpha_{2}^{\prime} z\left(t_{2}\right) \text {. }
$$

Subtraindo obtemos

$$
\alpha_{2}\left[z\left(t_{1}\right)-z\left(t_{2}\right)\right]=\alpha_{2}^{\prime}\left[z\left(t_{1}\right)-z\left(t_{2}\right)\right] .
$$

Daí, como $z\left(t_{1}\right)-z\left(t_{2}\right) \neq 0$, temos que $\alpha_{2}=\alpha_{2}^{\prime}$ e portanto $\alpha_{1}=\alpha_{1}^{\prime}$, donde

$$
g_{0}=h_{0} \text {. }
$$


Observação 4.2.13. Um caso particular do resultado obtido na proposição 4.2 .12 foi estudado no exemplo 1.2.6, o qual requereu um longo trabalho. Nele, foi necessário estudar varios casos, alguns deles bastante complicados. Observe-se que, utilizando o resultado anterior, resultam muito simples os cálculos.

Corolário 4.2.14. Para $\left.X=\left(C^{\prime} 0,1\right],\|\|_{\infty}\right)$ e $G=\left[1, t^{n}\right]$ temos que para qualquer $x \in X \backslash G$.

$$
R_{G}(x)=\left\{x(0)+[x(1)-x(0)] t^{n}\right\} .
$$

\section{Prova :}

Imediata. basta aplicar a proposição 4.2.12. 


\subsection{Uma Caracterização para Espaços Estritamen- te Convexos}

O objetivo desta seção é caracterizar os espaços estritamente convexos por meio da melhor coaproximação. Compararemos os resultados com outros já conhecidos sobre caracterização de espaços estritamente convexos utilizando a melhor aproximação. capítulo.

Daremos também, alguns exemplos e contraexemplos utilizando o assunto do

Definição 4.3.1. Um espaço normado $X$ é chamado estritamente convexo se para quaisquer $x, y \in X$ distintos com $\|x\|=\|y\|=1$, temos

$$
\left\|\frac{x+y}{2}\right\|<1 \text {. }
$$

Exemplo 4.3.2. Os espaços $L_{p}[0,1]$, os $l_{p}$ com suas normas $p$ correspondentes são estritamente convexos para $1<p<\infty$.

Exemplo 4.3.3. Os espaços $L_{1}[0,1], l_{1}, L_{\infty}[0,1]$ e $l_{\infty}$ com suas normas correspondentes não são estritamente convexos.

Exemplo 4.3.4. O espaço $\left(C[0,1],\|\|_{\infty}\right)$ não é estritamente convexo. De fato, sendo $x(t)=t$ e $y(t)=t^{2}$, temos que

$$
\|x\|_{\infty}=\sup _{t \in[0,1]}|x(t)|=1 \text { e }\|y\|_{\infty}=\sup _{t \in[0,1]}|y(t)|=1
$$

mas

$$
\left\|\frac{x+y}{2}\right\|_{\infty}=\sup _{t \in[0,1]}\left|\frac{x+y}{2}(t)\right|=1 .
$$




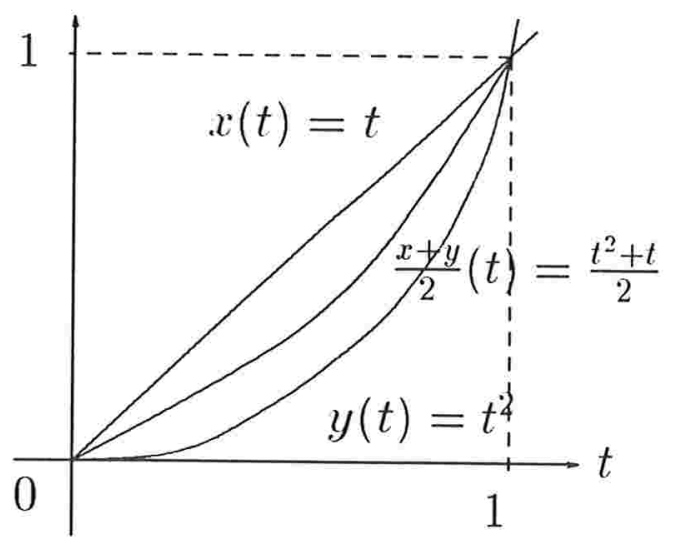

Figura 4.6. $\left(C[0,1],\|\|_{\infty}\right)$ não é estritamente convexo.

Observação 4.3.5. De acordo com a definição 4.3.1, deduz-se que um espaço normado é estritamente convexo se, e somente se, todo subespaço de dimensão 2 o é. Daí, por exemplo, $\left(\mathbb{R}^{2},\|\|_{p}\right)$ é estritamente convexo para $1<p<\infty$, pois os espaços $l_{p}$ o são. Os espaços $l_{1}$ e $l_{\infty}$ não são estritamente convexos pois $\left(\mathbb{R}^{2},\|\|_{1}\right)$ e $\left(\mathbb{R}^{2},\|\|_{\infty}\right)$, não o são.

Agora demonstraremos o resultado principal desta seção e logo vamos comparalo com os resultados conhecidos que vinculam convexidade estrita e melhor aproximaçào.

Teorema 4.3.6. Seja X um espaço normado. Então as seguintes afirmações são equivalentes:

(i) Para todo subespaço $G$ de $X$, todo $x \in X \backslash G$, todo $g_{0} \in R_{G}(x)$ e todo $g \in G$, temos

$$
\left\|g_{0}-g\right\|<\|x-g\|
$$

(ii) para todo subespaço $G$ de $X$ de dimensão 1 , todo $x \in X \backslash G$, todo $g_{0} \in R_{G}(x)$ e todo $g \in G$, temos

$$
\left\|g_{0}-g\right\|<\|x-g\|
$$

(iii) para todo subespaço $G$ de $X$, todo $x \in X^{\prime} \backslash G$ e todo $g_{0} \in R_{G}(x)$, temos

$$
\left\|g_{0}\right\|<\|x\|
$$


(iv) para todo subespaço $G$ de $X$ de dimensaio 1 , todo $x \in X \backslash G$ e todo $g_{0} \in R_{G}(x)$, temos

$$
\left\|g_{0}\right\|<\|x\|
$$

(v) X é estritamente convexo.

\section{Demonstração :}

Algumas das implicações são imediatas: (i) $\Rightarrow$ (ii) $\Rightarrow$ (iv) e (i) $\Rightarrow$ (iii) $\Rightarrow$ (iv).

(iv) $\Rightarrow(v)$

Se $X$ não for estritamente convexo, existem $x, y \in X$ distintos tais que

$$
\|x\|=\|y\|=\left\|\frac{x+y}{2}\right\|=1 .
$$

Seja $\varphi: \mathbb{R} \rightarrow \mathbb{R}$, dada por $\varphi(\alpha)=\|y+\alpha(x-y)\|$. Provaremos que $\varphi$ é uma função convexa. Sejam $\alpha, \beta \in \mathbb{R}$ e $t \in] 0,1[$.

$$
\begin{gathered}
\varphi(t \alpha+(1-t) \beta)=\|y+(t \alpha+(1-t) \beta)(x-y)\|= \\
=\|(1-t) y+t y+(1-t) \beta(x-y)\|=\|t(y+\alpha(x-y))+(1-t)(y+\beta(x-y))\| \leq \\
\leq t\|y+\alpha(x-y)\|+(1-t)\|y+\beta(x-y)\|=t \varphi(\alpha)+(1-t) \varphi(\beta),
\end{gathered}
$$

Logo, $\varphi$ é convexa.

Agora, utilizando a convexidade da $\varphi$, vamos provar que $\|y+\alpha(x-y)\| \geq 1$, ou seja, $\varphi(\alpha) \geq 1$, para todo $\alpha \in \mathbb{R}$.
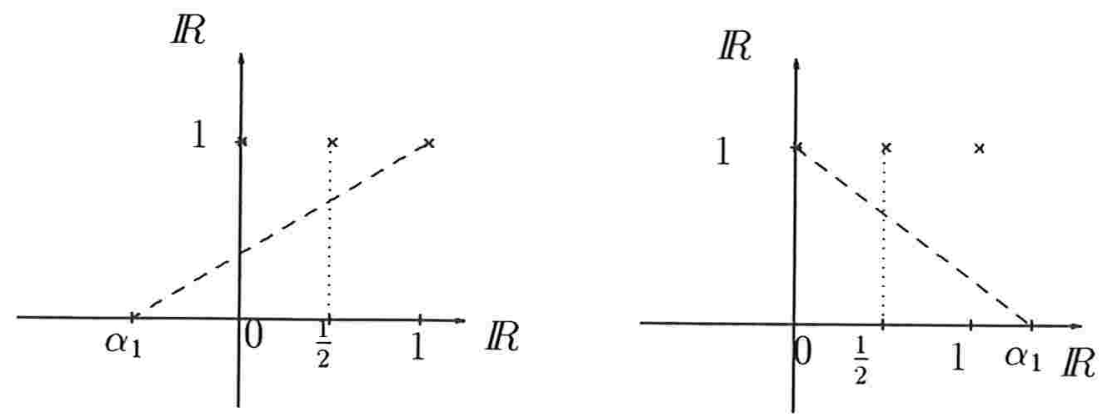

Figura 4.7. $\|y+\alpha(x-y)\| \geq 1$. 
Suponhamos que exista $\alpha_{1} \in \mathbb{R}$ tal que $\varphi\left(\alpha_{1}\right)<1$. Então $\alpha_{1} \notin\left\{0, \frac{1}{2}, 1\right\}$, pois $\varphi(0)=\varphi\left(\frac{1}{2}\right)=\varphi(1)=1$. O valor $\alpha=\frac{1}{2}$ pode ser obtido como combinação convexa de $\alpha_{1}$ e 1 ou de 0 e $\alpha_{1}$, dependendo da posição de $\alpha_{1}$ em relaçào a $\frac{1}{2}$ : $\alpha_{1}<\frac{1}{2}$ ou $\alpha_{1}>\frac{1}{2}$, respectivamente, (ver figura 4.7).

Como

$$
t \varphi\left(\alpha_{1}\right)+(1-t) \varphi(1)<1
$$

e

$$
t \varphi(0)+(1-t) \varphi\left(\alpha_{1}\right)<1
$$

e $\varphi\left(\frac{1}{2}\right)=1$, contrariamos o fato de $\varphi$ ser convexa. Logo, para todo $\alpha \in \mathbb{R}$,

$$
\|y+\alpha(x-y)\| \geq 1 .
$$

Usando (4.11), e como $\|y\|=1$, obtemos $y \perp(x-y)$.

Em resumo, tomando $G=[y]$ e $g_{0}=y$ obtivemos que $g_{0} \in R_{G}(x)$ com $\left\|g_{0}\right\|=\|x\|$ o que resulta absurdo pois, por hipótese, temos que $\left\|g_{0}\right\|<\|x\|$. Portanto $X$ é estritamente convexo.

(v) $\Rightarrow$ (i)

Suponhamos que existam um subespaço $G$ de $X, x \in X \backslash G, g_{0} \in R_{G}(x)$ e $g \in G$ tais que $\left\|g_{0}-g\right\|=\|x-g\|$. Pelo teorema 4.1.4 sabemos que existe $f=f^{g_{0}-g}$ em $X^{*}$ tal que

$$
\begin{gathered}
\|f\|=1, \\
f(x)=f\left(g_{0}\right), \\
f\left(g_{0}-g\right)=\left\|g_{0}-g\right\| .
\end{gathered}
$$

Daí obtemos

$$
f(x-g)=f\left(x-g_{0}+g_{0}-g\right)=f\left(g_{0}-g\right)=\left\|g_{0}-g\right\|=\|x-g\| .
$$

É fácil ver que $v_{1}=\frac{g_{0}-g}{\left\|g_{0}-g\right\|} \neq v_{2}=\frac{x-g}{\|x-g\|}$, pois $g_{0} \in G$ e $x \notin G$ e também que $g \neq g_{0}$ pois $x \neq g$.

Em resumo, obtivemos dois vetores distintos de norma $1, v_{1}$ e $v_{2}$, tais que $f$ atinge 
sua norma neles. Também temos que $f\left(\frac{v_{1}+v_{2}}{2}\right)=1$, onde $v_{1}+v_{2}$ não pode ser nulo pois $f \in X^{* *}$.

Se $\left\|\frac{v_{1}+v_{2}}{2}\right\|<1$, tomamos $\lambda=\frac{1}{\left\|\frac{v_{1}+v_{2}}{2}\right\|}>1$ e obtemos que $f\left(\lambda \frac{v_{1}+v_{2}}{2}\right)=\lambda>1$ o que é absurdo pela definição de norma de $f$.

Logo, $\left\|\frac{v_{1}+v_{2}}{2}\right\|=1$ e portanto $X$ nào é estritamente convexo, o que contradiz a hipótese.

Exemplo 4.3.7. Embora já tenha sido observado em 4.3.4, vamos ver, usando o teorema 4.3.6, que $X=\left(C[0,1],\|\|_{\infty}\right)$ não é estritamente convexo. Sejam $X=$ $\left(C[0.1],\|\|_{\infty}\right), G=[1, t]$ e $x=x(t)=t^{4}$. Pela proposição 4.2.12, sabemos que existe um único $g_{0} \in R_{G}(x)$ e ele é $g_{0}(t)=t$ (ver figura 4.8). É simples ver que as funçòes da forma $g_{\alpha}(t)=x-\alpha \operatorname{com} \alpha \geq \frac{3}{8 \sqrt[3]{2}}$ (ver exemplo 1.2.6) satisfazem que $\left\|g_{0}-g_{\alpha}\right\|=\left\|x-g_{\alpha}\right\|$.

Logo, $X=\left(C[0,1],\|\|_{\infty}\right)$ não é estritamente convexo.

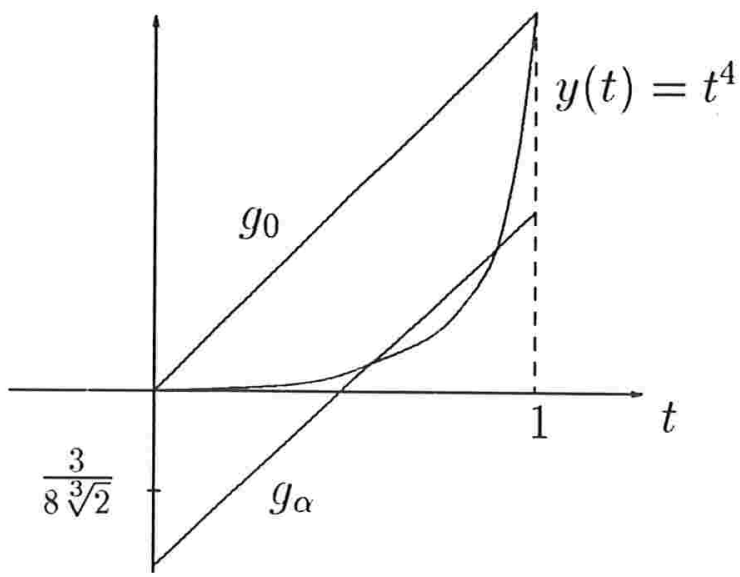

Figura 4.8.

A seguir citaremos um conhecido resultado que caracteriza espaços estritamente convexos através da melhor aproximação.

Teorema 4.3.8. Seja X um espaço normado. Então as seguintes afirmações são equivalentes

(i) Para todo subespaço $G$ de $X$, todo $x \in X \backslash G$, todo $g_{0} \in P_{G}(x)$ e todo $g \in$ $G \backslash\left\{g_{0}\right\}$, temos

$$
\left\|x-g_{0}\right\|<\|x-g\|
$$


(ii) para todo subespaço $G$ de dimensão 1 de $X$, todo $x \in X \backslash G$, todo $g_{0} \in P_{G}(x)$ e todo $g \in G^{\prime} \backslash\left\{g_{0}\right\}$, temos

$$
\left\|x-g_{0}\right\|<\|x-g\|
$$

(iii) X é estritamente convexo.

\section{Demonstração :}

O leitor interessado pode procurar uma prova deste resultado em [Sin70], página 110, corolário 3.3. 


\section{Capítulo 5}

\section{Propriedades do Operador $R_{C}$}

Em todo este capítulo, $X$ é um espaço normado real e estudaremos propriedades do operador $R_{C}$ no caso em que $C$ é um subconjunto convexo de $X$. No caso especial de $C$ ser um subespaço, utilizaremos como notação $R_{G}$. Também serão incluidas propriedades deste operador que foram estudadas em outros capítulos.

\subsection{Propriedades de $R_{G}$}

Nesta seção estudaremos propriedades do operador $R_{G}$, onde $G$ é um subespaço fechado de $X$.

Teorema 5.1.1. O operador $R_{G}$ tem as seguintes propriedades:

(i) Se $x \in D\left(R_{G}\right)$, então $\lambda x \in D\left(R_{G}\right)$ e $R_{G}(\lambda x)=\lambda R_{G}(x)$.

(ii) $G \subset D\left(R_{G}\right)$ e $R_{G}(x)=\{x\}$ para todo $x \in G$.

(iii) Sejam $x \in X$ e $g_{0} \in R_{G}(x)$. Então $\left\|g_{0}\right\| \leq\|x\|$.

(iv) Se $x \in D\left(R_{G}\right) \cap D\left(P_{G}\right)$, então $\left\|x-g_{0}\right\| \leq 2\left\|x-h_{0}\right\|$, para qualquer $g_{0}$ em $R_{G}(x)$ e qualquer $h_{0}$ em $P_{G}(x)$.

(v) $X$ é suave se e somente se $R_{G}(y)$ é unitário para todo y $\in D\left(R_{G}\right)$ e todo subespaço $G$ de $X$. Além disso, considerado como função. $R_{G}$ é linear. 
(vi) Se $X$ é suave, então $R_{G}$ é uma projeção de norma 1 de $D\left(R_{G}\right)$ em $G$.

(vii) Sejam Xr um espaço de Hilbert e $G$ um subespaço fechado de $X$. Então $D\left(R_{G}\right)=$ $X$ e $R_{G}=P_{G}$.

\section{Demonstração :}

(i) Basta utilizar a proposição 2.1.5.

(ii) É imediata pela definição de $R_{G}(x)$.

(iii) Como $0 \in G$, pois $G$ é subespaço, $R_{G}(x)$ é limitado por $\|x\|$.

(iv) Sejam $g_{0} \in R_{G}(x)$ e $h_{0} \in P_{G}(x)$. Pela proposição 2.2 .2 sabemos que

$$
\left(g_{0}-h_{0}\right) \perp\left(x-g_{0}\right),
$$

ou seja, para todo $\lambda \in \mathbb{R}$ vale

$$
\left\|g_{0}-h_{0}\right\| \leq\left\|g_{0}-h_{0}+\lambda\left(x-h_{0}\right)\right\| .
$$

Tomando $\lambda=1$ obtemos

$$
\left\|g_{0}-h_{0}\right\| \leq\left\|x-h_{0}\right\| .
$$

Além disso, pela desigualdade triangular temos

$$
\left\|x-g_{0}\right\| \leq\left\|x-h_{0}\right\|+\left\|g_{0}-h_{0}\right\| .
$$

Logo,

$$
\left\|x-g_{0}\right\| \leq 2\left\|x-h_{0}\right\|
$$

(v) Foi provado no teorema 2.4.4.

(vi) Por $(v), R_{G}$ pode ser considerado como função e é linear. É fácil ver que para todo $x \in D\left(R_{G}\right), R_{G}\left(R_{G}(x)\right)=R_{G}(x)$ e que para todo $x \in G, R_{G}(x)=x$, onde escrevemos $x$ e não $\{x\}$ por estarmos considerando $R_{G}$ como função. Logo, $R_{G}^{2}=R_{G}$.

Seja $x \in D\left(R_{G}\right)$. Por (iii) sabemos que

$$
\left\|R_{G}(x)\right\| \leq 1\|x\|
$$


e por (i) e (ii), se $x \in G$ temos que

$$
\left\|R_{G}(x)\right\|=1\|x\| .
$$

Portanto $R_{G}$ é contínua e

$$
\left\|R_{G}\right\|=1
$$

(vii) Foi provado na proposição 2.2.3.

Observação 5.1:2. Se $Y$ é um espaço vetorial e $Z$ é um subespaço de $Y$, não triviais, e $P: Y \rightarrow Z$ é uma funçào linear sobrejetora tal que $P^{2}=P$, então $Y=\operatorname{ker}(P) \oplus Z$ e $P(z)=z$ para todo $z \in Z$. Vejamos.

- Para todo $x \in Z$, existe $y \in Y \operatorname{com} P(y)=z$ e daí,

$$
z=P(y)=P^{2}(y)=P(P(y))=P(z) .
$$

- Se $y \in Y$, então $w=y-P(y) \in \operatorname{ker}(P)$, pois $P(w)=P(y)-P^{2}(y)=0$. Então $y=w+P(y) \in \operatorname{ker}(P)+Z$.

- Se $w \in \operatorname{ker}(P) \cap Y$, então $P(w)=0$ e $P(w)=w$, donde $w=0$.

Um caso particular, que usaremos no teorema 5.1.3, é o de uma projeção sobrejetora $P: G \oplus H \rightarrow G$, onde $G$ e $H$ são subespaços de um espaço de Banach $X$.

Outro, que usaremos no teorema 5.1.4, é o caso de uma projeção não nula $P: X \rightarrow V$, onde $X$ é um espaço de Banach e $V$ é um subespaço de dimensão 1.

Teorema 5.1.3. Se $x \in X \backslash G$, então as seguintes afirmações são equivalentes:

(i) $x \in D\left(R_{G}\right)$;

(ii) existe $P: G \oplus[x] \rightarrow G$ projeção sobrejetora de norma 1 .

\section{Demonstração :}

(i) $\Rightarrow$ (ii)

Seja $x \in D\left(R_{G}\right)$, ou seja, existe $g_{0} \in G_{r}$ tal que para todo $g \in G$

$$
\left\|g-g_{0}\right\| \leq\|x-g\| .
$$

Definimos $P: G \oplus[x] \rightarrow G$ por $P(g+\alpha x)=g+\alpha g_{0}$. Provaremos que $P$ satisfaz as condições desejadas. 
- $P(P(g+\alpha x))=P\left(g+\alpha g_{0}\right)=g+\alpha g_{0}=P(g+\alpha x)$.

Logo, $P^{2}=P$.

- $P\left[a\left(g_{1}+\alpha_{1} x\right)+b\left(g_{2}+\alpha_{2} x\right)\right]=P\left[\left(a g_{1}+b g_{2}\right)+\left(a \alpha_{1}+b \alpha_{2}\right) x\right]=$

$$
\begin{gathered}
a g_{1}+b g_{2}+\left(a \alpha_{1}+b \alpha_{2}\right) g_{0}=a\left(g_{1}+\alpha_{1} g_{0}\right)+b\left(g_{2}+\alpha_{2} g_{0}\right)= \\
=a P\left(g_{1}+\alpha_{1} x\right)+b P\left(g_{2}+\alpha_{2} x\right) .
\end{gathered}
$$

Logo, $P$ é linear.

- $P$ é claramente sobrejetora.

- Se $\alpha=0$ é óbvio que $\|P(g+\alpha x)\|=\|g+\alpha x\|$ pois $P(g)=g$.

Suponhamos $\alpha \neq 0$. Então, utilizando que $g_{0} \in R_{G}(x)$ e a parte (i) do teorema 5.1 .1 para $\alpha x$,

$$
\|P(g+\alpha x)\|=\left\|g+\alpha g_{0}\right\| \leq\|g+\alpha x\| .
$$

Logo, $\|P(g+\alpha x)\| \leq 1\|g+\alpha x\|$ e portanto $P$ é contínua com $\|P\| \leq 1$. Daí, por $(*)$,

$$
\|P\|=1 \text {. }
$$

(ii) $\Rightarrow$ (i)

Agora sabemos que existe $P: G \oplus[x] \rightarrow G$, projeção sobrejetora de norma 1. Provaremos que $P(x) \in R_{G}(x)$, ou seja, $\|P(x)-g\| \leq\|x-g\|$ para todo $g \in G$.

Como $P$ é sobrejetora, pela observação 5.1.2, $P(g)=g$ para todo $g \in G$. Além disso, como $P$ é linear.

$$
\|P(x)-g\|=\|P(x-g)\| .
$$

Sendo $\|P\|=1$,

$$
\|P(x-g)\| \leq\|P\|\|x-g\|=\|x-g\| .
$$

Como $P(x) \in G . P(x) \in R_{G}(x)$ e portanto

$$
x \in D\left(R_{G}\right) .
$$


Teorema 5.1.4. Dados X um espaço normado e $V$ subespaço de dimensão 1 de X, existe $P: X \rightarrow V$ projeção de norma 1 .

\section{Demonstração :}

Seja $v \in V,\|v\|=1$. Então $V=[v]$. Por Hahn-Banach, existe $f \in X^{*},\|f\|=1 \mathrm{e}$ $f(v)=\|v\|=1$.

Seja $H=\operatorname{ker}(f)$. Então $H$ é um hiperplano fechado. Afirmamos que $X=H \oplus V$. De fato, dado $x \in X$, consideremos $y_{x}=x-f(x) v$. Então $y_{x} \in H$, pois

$$
f\left(y_{x}\right)=f(x)-f(x) f(v)=0 .
$$

Assim, temos $x=(x-f(x) v)+f(x) v=y_{x}+f(x) v$ e $x \in H+V$.

A soma é direta pois se $z \in H \cap V$, então $f(z)=0$ e $z=\alpha v$ para algum $\alpha \in \mathbb{R}$, donde $f(z)=\alpha=0$. Portanto $z=0$, ou seja, $H \cap V=\{0\}$.

Definimos $P: X \rightarrow V$ por $P(x)=f(x) v$. Temos que $P$ é linear e também contínua pois $f \in X^{*}$. Além disso, para todo $x \in X$,

$$
P^{2}(x)=P(P(x))=P(f(x) v)=f(f(x) v) v=f(x) f(v) v=f(x) v=P(x) .
$$

Portanto $P$ é projeção.

Pela continuidade de $P$, para qualquer $x \in X$,

$$
\|P(x)\|=\left\|P^{2}(x)\right\| \leq\|P\|\|P(x)\| .
$$

Mas $P \not \equiv 0$ pois $P(v)=v \neq 0$ e então $\|P\| \geq 1$.

Por outro lado,

$$
\|P(x)\|=|f(x)|\|v\| \leq\|f\|\|x\|=\|x\|,
$$

e portanto,

$$
\|P\| \leq 1
$$

Assim,

$$
\|P\|=1 .
$$

Teorema 5.1.5. Seja $V$ um subespaço de dimensão 1 de X. Então

(i) $D\left(R_{V}\right)=X$; 
(ii) $R_{V}$ admite seletor linear.

\section{Demonstração :}

(i) Foi provado no teorema 2.2.5.

(ii) Pelo teorema 5.1.4 sabemos que existe $P: X \rightarrow V$ projeção de norma 1 , ou seja, $P$ é linear contínua, $P^{2}=P$ e $\|P\|=1$.

Provaremos que $P$ é um seletor de $R_{V}$.

Seja $x \in X$ qualquer. Vamos provar que $P(x) \in R_{V}(x)$ mostrando que $V \perp x-P(x)$.

Seja $v \neq 0$ tal que $[v]=V$. Tomemos $y=v+\lambda(x-P(x))$ com $\lambda \in \mathbb{R}$ qualquer. Como $\|P\|=1$ temos que $\|P(y)\| \leq\|y\|$. Logo, usando a linearidadade de $P$ e a observação 5.1.2,

$$
\|P(y)\|=\|P(v+\lambda(x-P(x)))\|=\left\|P(v)+\lambda\left(P(x)-P^{2}(x)\right)\right\|=\|P(v)\|=\|v\| .
$$

Portanto,

$$
\|v\| \leq\|v+\lambda(x-P(x))\| \text { para todo } \lambda \in \mathbb{R},
$$

o que por definição significa que $v \perp x-P(x)$.

Entào,

$$
V \perp x-P(x) .
$$

No lema 2.2.8, vimos que dados $G$ e $H$ subespaços de um espaço normado $X$ tais que $G \subset H$, se $x \in X \backslash H$ tem melhor coaproximação em $H$ e todo elemento de $H$ tem melhor coaproximação em $G$, então $x$ tem melhor coaproximação em $G$. Mais precissamente, se $h_{0} \in R_{H}(x)$ e $g_{0} \in R_{G}\left(h_{0}\right)$, então $g_{0} \in R_{G}(x)$. Em outras palavras, $R_{G}\left(R_{H}(x)\right) \subset R_{G}(x)$. No seguinte exemplo veremos que esta inclusão pode ser estrita.

Exemplo 5.1.6. Sejam $X=\left(C[0,1],\|\|_{\infty}\right), G=[1], H=[1, t]$ e $x(t)=3 t-2 t^{2}$. 


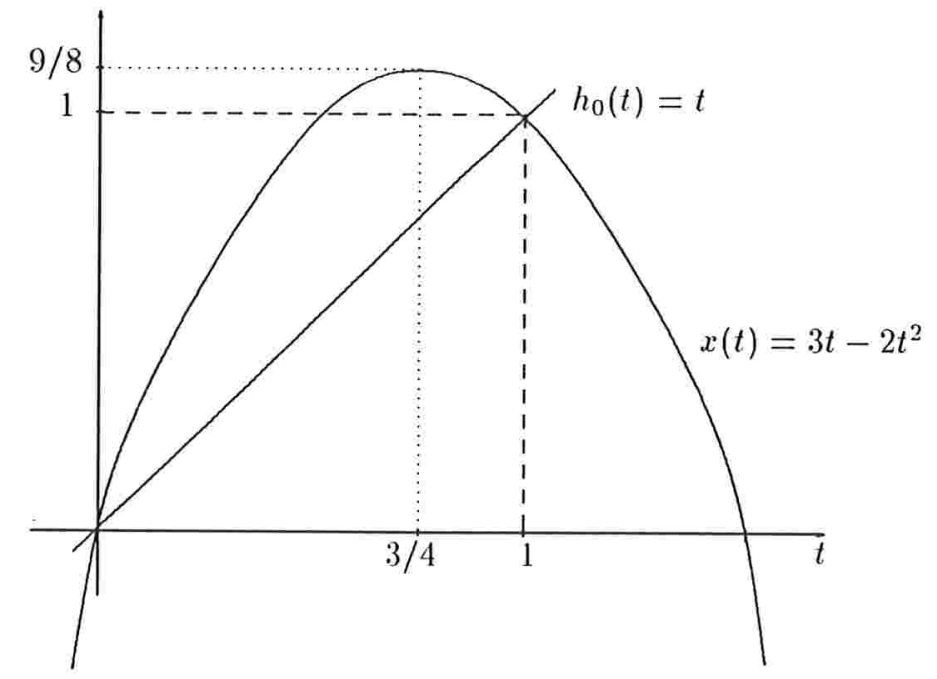

Figura 5.1.

Pela proposição 4.2 .12 sabemos que $R_{H}(x)=\left\{h_{0}\right\}$, onde $h_{0}(t)=t$. Agora vamos achar $R_{G}\left(R_{H}(x)\right)$, ou seja, $R_{G}\left(h_{0}\right)$.

Pela proposição 4.2 .9 , temos,

$$
R_{G}\left(h_{0}\right)=\left\{g_{0} \in G: g_{0} \equiv d, \text { com } 0 \leq d \leq 1\right\}
$$

e

$$
R_{G}(x)=\left\{g_{0} \in G: g_{0} \equiv c, \quad \text { com } 0 \leq c \leq \frac{9}{8}\right\} .
$$

Esquematicamente,

$$
R_{G}\left(h_{0}\right)=[0,1] \subsetneq\left[0, \frac{9}{8}\right]=R_{G}(x) .
$$

A seguir veremos que uma condição para a igualdade é $X$ ser suave.

Teorema 5.1.7. Sejam $X$ um espaço normado suave, $G$ e $H$ subespaços de $X$ tais que $G \subset H$. Então

(i) $R_{G}\left(R_{H}(x)\right)=R_{G}(x)$, para todo $x \in D\left(R_{H}\right)$;

(ii) $\left\|R_{G}(x)\right\| \leq\left\|R_{H}(x)\right\|$, para todo $x \in D\left(R_{H}\right) \cap D\left(R_{G}\right)$.

Demonstração : 
(i) Pelo lema 2.2.8 só precisamos mostrar que $R_{G}(x) \subset R_{G}\left(R_{H}(x)\right)$.

Seja $x \in D\left(R_{H}\right)$. Se $R_{G}(x)=\emptyset$, é óbvio que $R_{G}(x) \subset R_{G}\left(R_{H}(x)\right)$. Consideremos entào $g_{0} \in R_{G}(x)$. Provaremos que $g_{0} \in R_{G}\left(R_{H}(x)\right)$.

Como $X$ é suave, pelo teorema 2.4.4 podemos escrever $R_{H}(x)=\left\{h_{0}\right\}$, e temos $H \perp x-h_{0}$. Como $G \subset H$, também vale $G \perp x-h_{0}$. Além disso, $G \perp x-g_{0}$. Logo, pela proposição 2.1.5 e o teorema 2.4.2, temos

$$
G \perp\left[\left(x-g_{0}\right)-\left(x-h_{0}\right)\right]=h_{0}-g_{0} .
$$

Daí,

$$
g_{0} \in R_{G}\left(R_{H}(x)\right),
$$

obtendo o resultado desejado.

(ii) Pelo teorema 5.1.1 sabemos que $R_{G}$ é uma projeção de norma 1. Utilizando a parte (i), obtemos

$$
\left\|R_{G}(x)\right\|=\left\|R_{G}\left(R_{H}(x)\right)\right\| \leq\left\|R_{G}\right\|\left\|R_{H}(x)\right\|=\left\|R_{H}(x)\right\| .
$$

Observação 5.1.8. No teorema anterior é necessária a hipótese $x \in D\left(R_{H}\right)$ no ítem (i). De fato, considerando o espaço $X=\left(\mathbb{R}^{3},\|\|_{3}\right)$, existem subespaços $H$ de dimensào 2 para os quais $D\left(R_{H}\right)=H$, como vimos no capítulo 2 . Isso é devido ao fato de $X$ não ser Hilbert. Tomemos um desses $H_{\prime_{s}}$ e um seu subespaço $G$ de dimensão 1. Pelo teorema 2.2.5, $D\left(R_{G}\right)=X$ e portanto não está satisfeita a inclusão (i). 


\subsection{Caracterizações de espaços de Hilbert}

Nesta seção veremos mais duas caracterizações de espaços de Hilbert usando propriedades do operador $R_{G}$. Utilizaremos a notaçào $r_{G}$ para representar um seletor de $R_{G}$.

Definição 5.2.1. Sejam $G$ e $H$ dois subespaços de um espaço normado $X$. Dizemos que $G$ é $X$-isomorfo a $H$ se existir um isomorfismo $T: X \rightarrow X$ tal que $T(G)=H$.

No próximo lema veremos um tipo de isomorfismo que será utilizado no lema 5.2.3 e no teorema 5.2.4.

Lema 5.2.2. Sejam $X$ um espaço normado, $f \in X^{*} e z \in \operatorname{ker}(f)$.

Então $T: X \rightarrow X$, dado por $T(x)=x+f(x) z$ é um isomorfismo de $X$ em $X e$ $\left.T\right|_{\text {ker }(J)}$ é a identidade.

Prova :

- $T$ é obviamente linear.

- $T$ é injetora: Seja $x \in X$ tal que $T(x)=0$, ou seja,

$$
x+f(x) z=0,
$$

ou ainda,

$$
x=-f(x) z .
$$

Da igualdade acima, deduz-se que $x \in \operatorname{ker}(f)$ e daí $f(x)=0$ e $x=0$.

- $T$ é sobrejetora: Seja $y \in X$ e consideremos $x=y-f(y) z$. Provaremos que $T(x)=y$.

$$
\begin{gathered}
T(x)=T(y-f(y) z)=y-f(y) z+f(y-f(y) z) z= \\
=y-f(y) z+f(y) z-f(y) f(z) z=y .
\end{gathered}
$$

- $T$ é contínua:

$$
\|T(x)\|=\|x+f(x) z\| \leq\|x\|+\|f\|\|x\|\|z\|=\|x\|(1+\|f\|\|z\|) .
$$


- $T^{-1}$ é obviamente contínua: $T^{-1}(y)=y-f(y) z$ tem a mesma forma que $T$.

Lema 5.2.3. Sejam $X^{\prime}$ um espaço normado $\epsilon \varphi, \psi \in m . X^{*}$ não nulos. Então ker $(\varphi)$ é $X$ - isomorfo a $\operatorname{ker}(\psi)$.

\section{Prova :}

Se $\operatorname{ker}(\varphi)=\operatorname{ker}(\psi)$, entào a identidade satisfaz as condições.

Se $\operatorname{ker}(\varphi) \neq \operatorname{ker}(\psi)$, consideremos $z \in \operatorname{ker}(\psi,-\varphi)$, tal que $z \notin \operatorname{ker}(\varphi) \cup \operatorname{ker}(\psi)$. Vejamos que tal elemento existe. Suponhamos que $\operatorname{ker}(\psi-\varphi) \subset \operatorname{ker}(\varphi) \cup \operatorname{ker}(\psi)$ e seja $z_{0} \in \operatorname{ker}(\psi-\varphi)$. Se $z_{0} \in \operatorname{ker}(\varphi)$, então $z_{0} \in \operatorname{ker}\left(\iota^{\circ}\right)$ e reciprocamente. Portanto,

$$
\operatorname{ker}(\psi-\varphi) \subset \operatorname{ker}(\varphi) \cap \operatorname{ker}(\psi) .
$$

Mas isso é absurdo pois $\operatorname{ker}(\varphi) \cap \operatorname{ker}(\psi)$ é um subespaço de codimensão 2.

Seja $\bar{z}=\frac{1}{\varphi(z)} z$. Observemos que $\varphi(\bar{z})=\psi(\bar{z})=1$ e que $\bar{z} \in \operatorname{ker}(\psi-\varphi)$.

Tomemos um operador $T: X \rightarrow X$ definido por $T(x)=x+(\psi(x)-\varphi(x)) \bar{z}$. Pelo lema 5.2 .2 sabemos que $T$ é um isomorfismo de $X$ em $X$ que deixa fixo $\operatorname{ker}(\psi-\varphi)$. Resta provar que $T$ leva $\operatorname{ker}(\psi)$ em $\operatorname{ker}(\varphi)$.

Seja $w \in \operatorname{ker}(\psi)$. Então $T(w)=w-\varphi(w) \bar{z}$ e $T(w) \in \operatorname{ker}(\varphi)$ pois,

$$
\varphi(T(w))=\varphi(w)-\varphi(w) \varphi(\bar{z})=0 .
$$

Logo,

$$
T(\operatorname{ker}(\psi)) \subset \operatorname{ker}(\varphi) \text {. }
$$

Seja agora $u \in \operatorname{ker}(\varphi)$. Como $X=\operatorname{ker}(\psi) \oplus[\bar{z}]$, pois $\bar{z} \notin \operatorname{ker}(\psi)$, podemos escrever $u=w_{0}+\lambda \bar{z}$, para algum $w_{0} \in \operatorname{ker}(\psi)$ e algum $\lambda \in \mathbb{R}$. Observemos que $\varphi\left(w_{0}\right)=-\lambda$, pois $\varphi\left(w_{0}\right)=0$ e $\varphi(\bar{z})=1$. Então,

$$
T\left(w_{0}\right)=w_{0}+\left[\psi\left(w_{0}\right)-\varphi\left(w_{0}\right)\right] \bar{z}=w_{0}+\lambda \bar{z}=u
$$

e portanto $u \in T(k e r(\psi))$.

Logo,

$$
\operatorname{ker}(\varphi) \subset T(\operatorname{ker}(\psi)) \text { e } T\left(\operatorname{ker}\left(\psi^{\prime}\right)\right)=\operatorname{ker}(\varphi)
$$


Teorema 5.2.4. Seja X um espaço de Banach de dimensão maior ou igual a 3. Então, as seguintes afirmações são equivalentes:

(i) X é um espaço de Hilbert;

(ii) existe $Y$, subespaço fechado próprio e não trivial de $X$, tal que para todo subespaço fechado $H$ de $X, X$-isomorfo a $Y$ e para todo $x \in X$, temos que se $g_{1} \in P_{H}(x)$ então $\left\|g_{1}\right\| \leq\|x\|$;

(iii) para todo subespaço $L$ de $X$ de dimensão 1 e todo $x \in X$, temos que se $y \in P_{L}(x)$ então $\|y\| \leq\|x\|$.

\section{Demonstração :}

(i) $\Rightarrow$ (ii)

É imediato, pelo teorema de Pitágoras, para quaisquer $Y, H$ e $x$.

(ii) $\Rightarrow$ (iii)

Sejam $x \in X, L$ um subespaço de $X$ de dimensão 1 e $x_{0} \in L$ tal que $\left\|x_{0}\right\|=1$. Sem perda de generalidade, pela homogeneidade da melhor aproximação, consideraremos $\|x\|=1$. Seja $y \in P_{L}(x)$. Provaremos que $\|y\| \leq\|x\|$.

Se $x \in L$, então $y=x$ e nada temos a provar.

Se $x \notin L$, então $d(L, x)=\|x-y\|=d>0$.

Tomemos agora o subespaço $Y$ da hipótese. Para $\bar{x} \in Y$, não nulo, existe, pelo teorema de Hann-Banach, $f \in X^{*}$ tal que $f\left(x_{0}\right)=f(\bar{x})=1$. Consideremos o operador $K: X \rightarrow X$ definido por $K(z)=z+f(z)\left(x_{0}-\bar{x}\right)$. Pelo lema 5.2.2, temos que $K$ é um isomorfismo de $X$ em $X$. Vejamos que $K$ leva $[\bar{x}]$ em $L=\left[x_{0}\right]$.

$$
K(\bar{x})=\bar{x}+f(\bar{x})\left(x_{0}-\bar{x}\right)=\bar{x}+\left(x_{0}-\bar{x}\right)=x_{0} .
$$

Chamemos $G=K(Y)$. Como $K$ é um isomorfismo de $X$ em $X$, então $G$ é $X$-isomorfo a $Y$, e ainda $x_{0} \in G$.

Por outro lado, temos que a distância de $x$ a $L=\left[x_{0}\right]$ é d e $\|x-y\|=d$. Então, como $x_{0}$ e $x-y$ são linearmente independentes, pelo teorema de Hann-Banach, existe $\varphi \in X^{*}$ tal que $\varphi\left(x_{0}\right)=0,\|\varphi\|=1$ e $\varphi(x-y)=d$. Consideremos também $\psi \in X^{*}$ que se anule em $G$ (e portanto $G \subset \operatorname{ker}(\psi)$ ) com $\|\psi\|=1$. Pelo lema 5.2.3 sabemos que existe um isomorfismo $T: X \rightarrow X$ que leva $\operatorname{ker}(\psi)$ em $\operatorname{ker}(\varphi)$. Seja $H=T(G)$. É fácil ver que $H \subset \operatorname{ker}(\varphi)$. Como $T \circ K$ é um isomorfismo de $X$ em $X$ que leva $Y$ em $H$, temos que $H$ também é $X$-isomorfo a $Y$. 
Além disso, $x=y+(x-y)$, e daí $\varphi(x)=d$, pois $y \in L,\left.\varphi\right|_{L}=0$ e $\varphi(x-y)=d$. Portanto,

$$
\|z-x\| \geq|\varphi(z-x)|=d \text { para todo } z \in \operatorname{ker}(\varphi) .
$$

Isso implica que a distância de $x$ a $H$ é $d$ pois $y \in L \subset H$ atinge tal distância, donde,

$$
y \in P_{H}(x),
$$

o que por hipótese implica em

$$
\|y\| \leq\|x\|
$$

(iii) $\Rightarrow$ (i)

Para provar que $X$ é um espaço de Hilbert, usaremos o teorema 2.2.12, isto é, demonstraremos que dados $x, y$ em $X$ tais que $x \perp y$, tem-se que $y \perp x$. Sem perda de generalidade, pela homogeneidade da ortogonalidade, tomaremos $x$ e $y$ tais que $\|x\|=\|y\|=1$.

Sejam então, $x$ e $y$ em $X$ tais que $x \perp y$ e $\|x\|=\|y\|=1$. Provaremos que $y \perp x$, ou seja, $\|y-t x\| \geq\|y\|=1$ para todo $t \in \mathbb{R}$.

Seja $x_{0} \in P_{[x]}(y)$, ou seja, $\inf _{t \in R}\|y-t x\|=\left\|y-x_{0}\right\|$. Suponhamos que $\left\|y-x_{0}\right\|<$ $1=\|y\|$.

Como $x \perp y$, pela homogeneidade da ortogonalidade, temos que $x_{0} \perp y$. Portanto,

$$
\left\|x_{0}+k y\right\| \geq\left\|x_{0}\right\| \text { para todo } k \in \mathbb{R} .
$$

Expressando (5.1) convenientemente obtemos

$$
\inf _{t \in R}\left\|\left(x_{0}-y\right)-t y\right\| \geq\left\|\left(x_{0}-y\right)-(-y)\right\| .
$$

Mas, essa última desigualdade significa que $(-y) \in P_{[y]}\left(x_{0}-y\right)$. Então, por hipótese,

$$
\|-y\| \leq\left\|x_{0}-y\right\|
$$

e daí

$$
\|y\|<1 \text {, o que resulta absurdo. }
$$

Logo, pelo teorema 2.2.12, $\mathrm{X}$ é um espaço de Hilbert. 
Definição 5.2.5. Sejam $X$ e $Z$ espaços normados e $f: X \rightarrow Z$. Dizemos que $f$ é contrativa se

$$
\|f(x)-f(y)\| \leq\|x-y\| \text { para quaisquer } x, y \in X \text {. }
$$

A seguir veremos algumas caracterizações de espaços de Hilbert que utilizam a parte (iii) do teorema 5.2.4.

Teorema 5.2.6. Seja $X$ um espaço de Banach de dimensão maior ou igual que 3. Então, as seguintes afirmações são equivalentes:

(i) X' é um espaço de Hilbert;

(ii) $X$ é estritamente convexo e para todo subespaço fechado $G$ de $X$, temos que $D\left(R_{G}\right)=X$ e $\left(I-r_{G}\right)$ é contrativa para qualquer $r_{G}$ seletor de $R_{G}$;

(iii) X é estritamente convexo e para todo subespaço $L$ de $X$ de dimensão 1 , temos que $I-r_{L}$ é contrativa para qualquer $r_{L}$;

(iv) para todo subespaço $L$ de $X$ de dimensão 1 e todo $x \in X$, temos que

$$
P_{L}(x)=R_{L}(x)
$$

(v) para todo subespaço $L$ de $X$ de dimensão 1 e todo $x \in X$, temos que

$$
P_{L}(x) \subset R_{L}(x)
$$

\section{Demonstração :}

(i) $\Rightarrow$ (ii)

Como $X$ é um espaço de Hilbert, é estritamente convexo. Seja $G$ um subespaço fechado de $X$. Pela proposição 2.2.14, temos que $D\left(R_{G}\right)=X$. Só resta provar que $\left(I-r_{G}\right)$ é contrativa.

Pela proposição 2.2.3, sabemos que $R_{G}=P_{G}$ e portanto $r_{G}$ é a projeção ortogonal sobre $G$. Então, para $x, y \in X$ temos

$$
\left\|\left(I-r_{G}\right)(x)-\left(I-r_{G}\right)(y)\right\|=\left\|(x-y)-r_{G}(x-y)\right\| \leq\|x-y\| .
$$

Logo,

$$
\left(I-r_{G}\right) \text { é contrativa. }
$$


(ii) $\Rightarrow$ (iii)

A implicação é óbvia.

(iii) $\Rightarrow($ iv $)$

Seja $L$ um subespaço de $X$ de dimensão 1 . Notemos que $D\left(R_{L}\right)=X$ pois $L$ tem dimensão 1 (teorema 2.2.5). Tomemos $x \in X, g_{0} \in R_{L}(x)$ e $g \in L$. Usando um seletor $r_{L}$ de $R_{L}$ com $r_{L}(x)=g_{0}$, que $\left(I-r_{L}\right)$ é contrativa e que $r_{L}$ deixa fixos os elementos de $L$, temos

$$
\begin{gathered}
\left\|x-g_{0}\right\|=\left\|x-r_{L}(x)+g-r_{L}(g)\right\|= \\
=\left\|\left(I-r_{L}\right)(x)-\left(I-r_{L}\right)(g)\right\| \leq\|x-g\| .
\end{gathered}
$$

Portanto,

$$
\left\|x-g_{0}\right\| \leq\|x-g\| \text { para todo } g \in L,
$$

ou seja,

$$
g_{0} \in P_{L}(x)
$$

Logo,

$$
R_{L}(x) \subset P_{L}(x)
$$

Como $X$ é estritamente convexo, pelo corolário 3.3, pag. 110 de [Sin70], a melhor aproximaçào sobre $L$ é única. Mas $R_{L}(x) \neq \emptyset(2.2 .5)$ e então

$$
P_{L}(x)=R_{L}(x) .
$$

(iv) $\Rightarrow(v)$

A implicaçào é óbvia.

(v) $\Rightarrow$ (i)

Seja $L$ um subespaço de $X$ de dimensão 1 . Então $D\left(R_{L}\right) \neq \emptyset$. (2.2.5)

Consideremos $x \in D\left(P_{L}\right)$ e tomemos $g_{1} \in P_{L}(x)$. Como $P_{L}(x) \subset R_{L}(x), g_{1} \in R_{L}(x)$ e portanto

$$
\left\|g_{1}-g\right\| \leq\|x-g\| \text { para todo } g \in G .
$$

Logo, tomando $g=0$,

$$
\left\|g_{1}\right\| \leq\|x\|
$$


Pelo teorema 5.2.4, $X$ é um espaço de Hilbert.

Agora veremos outras caracterizações de espaços de Hilbert que utilizam o teorema 5.2.4, parte (ii).

Teorema 5.2.7. Seja X um espaço de Banach de dimensão maior ou igual a 3. Então, as seguintes afirmações são equivalentes:

(i) X é um espaço de Hilbert;

(ii) $X$ é estritamente convexo e existe um subespaço fechado própio $Y$ de $\mathrm{X}$ tal que para todo subespaço fechado $G, X$-isomorfo a $Y$, temos que $D\left(r_{G}\right)=X$ e $\left(I-r_{G}\right)$ é contrativa para qualquer $r_{G}$ seletor de $R_{G}$;

(iii) existe um subespaço fechado próprio $Y$ de $X$ tal que, para todo subespaço fechado $G X$-isomorfo a $Y$ e todo $x \in X$, temos que $P_{G}(x) \subset R_{G}(x)$.

\section{Demonstração :}

As demonstrações são análogas às do teorema 5.2.6. 


\subsection{Propriedades de $R_{C}$}

Nesta seção estudaremos o operador $R_{C}$ de melhor coaproximação em conjuntos convexos. Em toda a seção, $C$ é um subconjunto convexo de $X$.

Começamos com um exemplo interessante sobre melhor coaproximação em um conjunto convexo que generaliza o exemplo 1.2.2.

Exemplo 5.3.1. Sejam $X=\left(\mathbb{R}^{2},\|\|_{2}\right), C=\left\{\left(x_{1}, x_{2}\right) \in \mathbb{R}^{2}: x_{1}^{2}+x_{2}^{2} \leq 1\right\}$ e $x=(a, 0)$, onde $a \geq 1$. Calculemos $R_{C}(x)$.

Fazendo um raciocínio geométrico, podemos dizer que $c_{0} \in R_{C}(x)$ se, e somente se $C$ e $x$ estão em semiplanos opostos determinados pela mediatriz do segmento unindo $c_{0}$ a $x$. Destas retas, as "piores" são as tangentes a $C$.

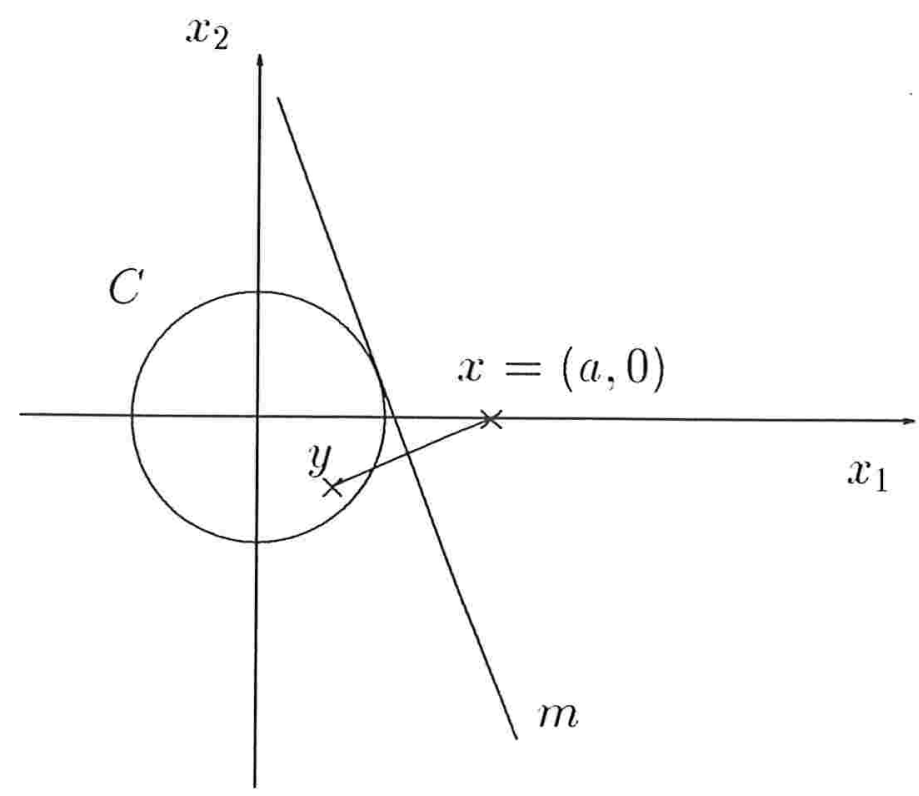

\section{Figura 5.2.}

Consideremos então só as retas $m$ tangentes a $C$ e seja y o simétrico de $x$ respeito de $m$. Resulta simples ver que para cada $m$, se $y \in C, C \cap[y, x] \subset R_{C}(x)$. 
A seguir. daremos a expressão paramétrica da curva $\gamma$ formada pelos pontos $y$, cujas operaçòes foram omitidas.

$$
\gamma(\theta)=\left(2 a \cos ^{2} \theta+2 \sin \theta-a, 2 \sin \theta-2 a \sin \theta \cos \theta\right) .
$$

A figura 5.3 representa o caso particular da curva $\gamma$ para $x=(1.8,0)$, onde a região quadriculada indica o conjunto $R_{C}(x)$.

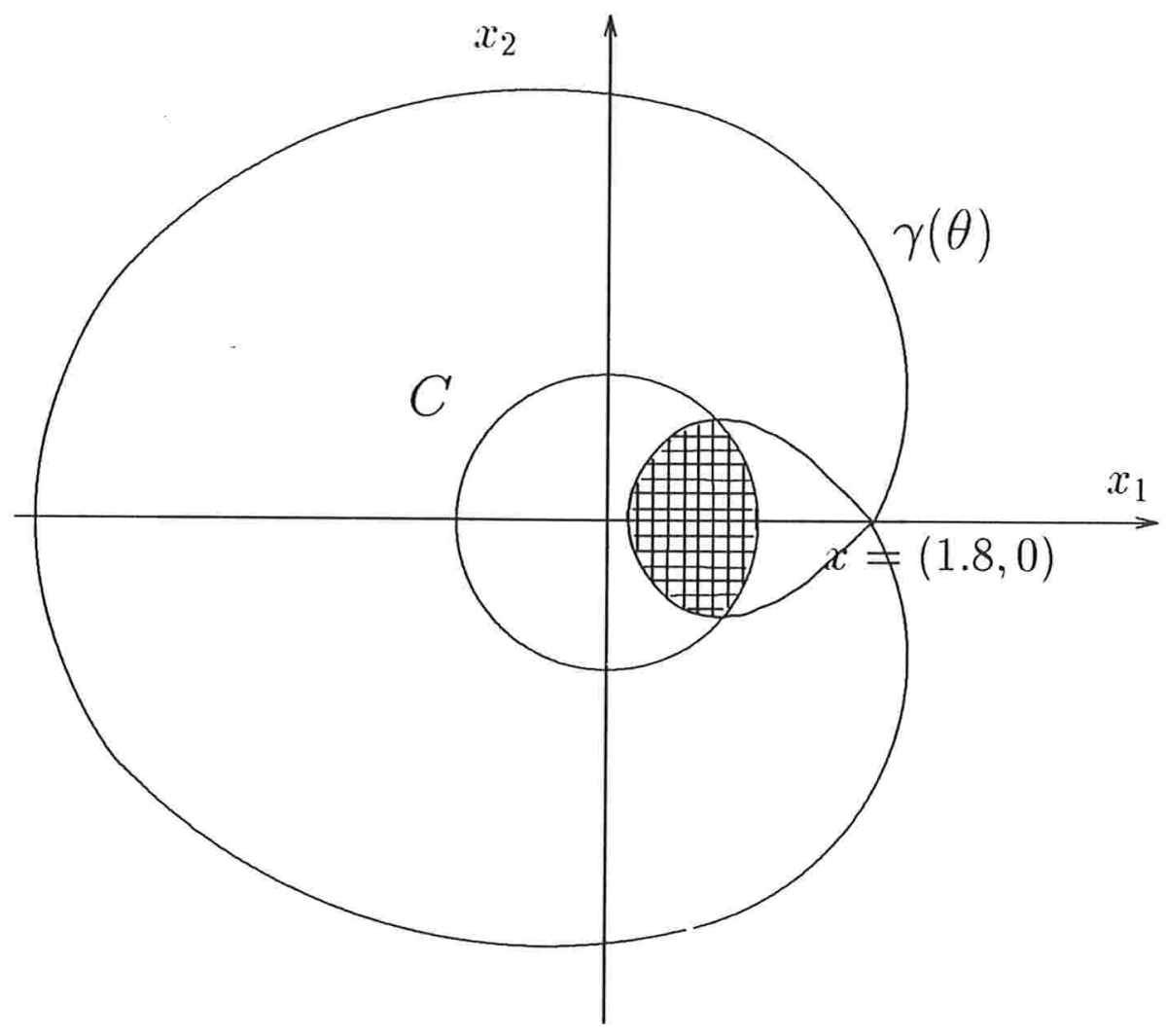

Figura 5.3.

Proposição 5.3.2. O operador $R_{C}$ tem as seguintes propriedades:

(i) $C \subset D\left(R_{C}\right)$ e $R_{C}(x)=\{x\}$ para todo $x \in C$.

(ii) Se Céfechado, então $R_{C}(x)$ é fechado, para todo $x \in X$.

(iii) Se $C$ é convexo, então $R_{C}(x)$ é convexo, para todo $x \in X$. 
(iv) $R_{C}(x)$ é limitado para todo $x \in X$.

(v) Se $x \in D\left(R_{C}\right)$ e $c_{0} \in R_{C}(x)$, então $c_{0} \in R_{C}\left(t x+(1-t) c_{0}\right)$, para todo $t \in[1,+\infty[$.

(vi) Se $x \in D\left(R_{C}\right)$ e $\operatorname{diam}(C) \leq \operatorname{dist}(x, C)$, então $C=R_{C}(x)$.

\section{Prova :}

As propriedades (i), (ii), (iii) e (iv) são nossas conhecidas: 1.1.2 e 1.1.5.

(v) Queremos provar que para todo $c \in C$ e todo $t \in[1,+\infty[$.

$\left\|c-c_{0}\right\| \leq\left\|t x+(1-t) c_{0}-c\right\|$.

Fazendo operações e utilizando o fato de que $c_{0} \in R_{C}(x)$, temos

$$
\begin{gathered}
\left\|t x+(1-t) c_{0}-c\right\|=\left\|t x+(1-t) c_{0}+c t-c-c t\right\|= \\
=\left\|t(x-c)+(1-t)\left(c_{0}-c\right)\right\| \geq\|t(x-c)\|-\left\|(1-t)\left(c_{0}-c\right)\right\|= \\
=|t|\|x-c\|-|1-t|\left\|c_{0}-c\right\| \geq|t|\left\|c_{0}-c\right\|-|1-t|\left\|c_{0}-c\right\|= \\
=(t+(1-t))\left\|c_{0}-c\right\|=\left\|c_{0}-c\right\| .
\end{gathered}
$$

(vi) Seja $c_{0} \in C$. Aplicando as definições de diâmetro e de distância,

$$
\left\|c-c_{0}\right\| \leq \sup _{z, y \in C}\|z-y\|=\operatorname{diam}(C) \leq \operatorname{dist}(x, C)=\inf _{y \in C}\|x-y\| \leq\|c-x\| .
$$

Logo, $C \subset R_{C}(x)$ e como $R_{C}(x) \subset C$, temos $C=R_{C}(x)$. 


\section{$5.4 R_{C}$ e a retração ortogonal}

Vamos definir a retração ortogonal, outra forma de aproximação introduzida em [Bru73] e relacioná-la com a melhor coaproximação.

Definição 5.4.1. Chamaremos funcional tangente à função $\tau: X \times X \rightarrow \mathbb{R}$ definida por

$$
\tau(x, y)=\lim _{t \rightarrow 0^{+}} \frac{\|x+t y\|-\|x\|}{t} .
$$

Observação 5.4.2. Este limite representa a derivada direcional pela direita no ponto $x$ na direção $y$ da função \|\| . O funcional tangente é um caso particular de $d^{+} f_{x}$ para $f(v)=\|v\|$, e portanto existe sempre (ver observação 2.3.2 e teorema 2.3.4).

Definição 5.4.3. Consideremos $C \subset X$ um subconjunto convexo. Sejam $x \in X$ e $c_{0} \in C$. Dizemos que $c_{0}$ pertence à retração ortogonal de $x$ em $C$, e indicamos $c_{0} \in Q_{C}(x)$, se

$$
\tau\left(c_{0}-c, x-c_{0}\right) \geq 0 \text { para todo } c \in C .
$$

Observação 5.4.4. Podemos escrever

$$
Q_{C}(x)=\left\{x \in C: \tau\left(c_{0}-c, x-c_{0}\right) \geq 0 \text { para todo } c \in C\right\} .
$$

Se $x \in C, \tau(x-c, 0)=0$, e portanto $x \in Q_{C}(x)$. Se $c_{0} \in Q_{C}(x)$ e $c_{0} \neq x$, tomamos $c=x$. Então,

$$
\tau\left(c_{0}-x, x-c_{0}\right)=\lim _{t \rightarrow 0^{+}} \frac{\left\|c_{0}-x+t\left(x-c_{0}\right)\right\|-\left\|c_{0}-x\right\|}{t}=-\left\|x-x_{0}\right\|<0 .
$$

Portanto $Q_{C}(x)=\{x\}$, quando $x \in C$.

Exemplo 5.4.5. Sejam $X=\left(\mathbb{R}^{2},\|\|_{2}\right), C=\left\{\left(x_{1}, x_{2}\right) \in \mathbb{R}^{2}: x_{1}^{2}+x_{2}^{2} \leq 1\right\} \mathrm{e}$ $x=(3,0)$. Veremos que $Q_{C}(x)=\{(1,0)\}$. 
Provemos primeiro que $(1,0) \in Q_{C}(x)$, ou seja, que $\tau\left(c_{0}-c, x-c_{0}\right) \geq 0$, onde $c=(a, b) \operatorname{com} a^{2}+b^{2} \leq 1, c_{0}=(1,0)$ e $x=(3,0)$.

$$
\begin{gathered}
\tau\left(c_{0}-c, x-c_{0}\right)=\tau((1-a,-b),(2,0))=\lim _{t \rightarrow 0^{+}} \frac{\|(1-a,-b)+t(2,0)\|-\|(1-a,-b)\|}{t}= \\
=\lim _{t \rightarrow 0^{+}} \frac{\|(1-a,-b)+t(2,0)\|^{2}-\|(1-a,-b)\|^{2}}{t(\|(1-a,-b)+t(2,0)\|+\|(1-a,-b)\|)}= \\
=\lim _{t \rightarrow 0^{+}} \frac{4 t(1-a+t)}{t(\|(1-a,-b)+t(2,0)\|+\|(1-a,-b)\|)}=\frac{1-a}{2\|(1-a,-b)\|} \geq 0 .
\end{gathered}
$$

Logo,

$$
c_{0} \in Q_{C}(x) .
$$

Agora provaremos que $c_{1}=(a, b) \in C$, com $(a, b) \neq(1,0)$, não pertence a $Q_{C^{\prime}}(x)$. Seja $c=(1,0)$. Então,

$$
\begin{gathered}
\tau\left(c_{1}-c, x-c_{1}\right)=\lim _{t \rightarrow 0^{+}} \frac{\left\|\left(c_{1}-c\right)+t\left(x-c_{1}\right)\right\|-\left\|c_{1}-c\right\|}{t}= \\
\lim _{t \rightarrow 0^{+}} \frac{\|(a-1+t(3-a), b(1-t))\|^{2}-\|(a-1, b)\|^{2}}{t\left(\left\|\left(c_{1}-c\right)+t\left(x-c_{1}\right)\right\|+\left\|c_{1}-c\right\|\right)}= \\
\lim _{t \rightarrow 0^{+}} \frac{t\left((3-a)(2(a-1)+t(3-a))+b^{2}(t-2)\right)}{t\left(\left\|\left(c_{1}-c\right)+t\left(x-c_{1}\right)\right\|+\left\|c_{1}-c\right\|\right)} .
\end{gathered}
$$

Logo,

$$
\tau\left(c_{1}-c, x-c_{1}\right)=-\frac{a^{2}+b^{2}-4 a+3}{\left\|c_{1}-c\right\|} .
$$

Definamos agora a função $f: C \subset \mathbb{R}^{2} \rightarrow \mathbb{R}$ por $f(a, b)=-\frac{a^{2}+b^{2}-4 a+3}{\left\|c_{1}-c\right\|}$. Depois de alguns cálculos que serão omitidos, conclui-se que $f(a, b)<0$ se $a^{2}+b^{2} \leq 1$ e $(a, b) \neq(1.0)$. Portanto,

$$
c_{1} \notin Q_{C}(x)
$$

e então

$$
Q_{C}(x)=\{(1,0)\} .
$$


Teorema 5.4.6. $Q_{C}(x) \subset R_{C}(x) \in Q_{C}(x) \subset Q_{C}\left(t x+(1-t) c_{0}\right)$ para todo $t \geq 0$.

\section{Demonstração :}

Seja $c_{0} \in Q_{C}(x)$. Então $\tau\left(c_{0}-c, x-c_{0}\right) \geq 0$, ou ainda,

$$
\lim _{t \rightarrow 0^{+}} \frac{\left\|c_{0}-c+t\left(x-c_{0}\right)\right\|-\left\|x-c_{0}\right\|}{t} \geq 0 . \text { para todo } c \in C .
$$

Como esse limite é atingido em forma decrescente (teorema 2.3.4), podemos afirmar que para $c \in C,\left\|c_{0}-c+t\left(x-c_{0}\right)\right\| \geq\left\|x-c_{0}\right\|$ para todo $t \geq 0$, e em particular para $t=1$.

Daí,

$$
\|x-c\| \geq\left\|x-c_{0}\right\| \text { para todo } c \in C,
$$

ou seja,

$$
c_{0} \in R_{C}(x) .
$$

Provemos agora que $c_{0} \in Q_{C}\left(t x+(1-t) c_{0}\right)$ para todo $t \geq 0$. Como para $t=0$ a afirmação é óbvia, podemos supor que $t>0$.

Aplicando a definição e fazendo algumas operações. temos

$$
\begin{gathered}
\tau\left(c_{0}-c, t x+(1-t) c_{0}-c_{0}\right)=\tau\left(c_{0}-c, t\left(x-c_{0}\right)\right)= \\
=\lim _{\lambda \rightarrow 0^{+}} \frac{\left\|c_{0}-c+\lambda\left[t\left(c-c_{0}\right)\right]\right\|-\left\|c_{0}-c\right\|}{\lambda}=\lim _{\lambda \rightarrow 0^{+}} \frac{\left.\| c_{0}-c+(\lambda t)\left(c-c_{0}\right)\right]\|-\| c_{0}-c \|}{\lambda t}= \\
=t \tau\left(c_{0}-c, x-c_{0}\right) \geq 0 .
\end{gathered}
$$

Teorema 5.4.7. Se C é um subespaço de X, então

$$
R_{C}=Q_{C} .
$$




\section{Demonstração :}

Se $C$ é um subespaço de $X$, sabemos que $c_{0} \in R_{C}(x)$ se, e somente se, $C \perp x-c_{0}$. Em particular,

$$
c_{0}-c \perp x-c_{0} \text { para todo } c \in C
$$

e daí.

$$
\left\|\left(c_{0}-c\right)+t\left(x-c_{0}\right)\right\| \geq\left\|c_{0}-c\right\| \text { para todo } x \in C \text { e todo } t \in \mathbb{R} .
$$

Portanto,

$$
\tau\left(c_{0}-c, x-c_{0}\right) \geq 0 \text { e } R_{C}(x) \subset Q_{C}(x) .
$$

Pelo teorema 5.4.6,

$$
R_{C}(x)=Q_{C}(x)
$$

Corolário 5.4.8. Se X é um espaço pré-hilbertiano e $C$ é um subespaço de $X$, então

$$
R_{C}=Q_{C}=P_{C} .
$$

\section{Prova :}

É conseqüência do teorema 5.4 .7 e da proposição 2.2.3.

Observação 5.4.9. Nos teoremas 5.4.6 e 5.4.7, provamos que $Q_{C}(x) \subset R_{C}(x)$ e que são iguais se $C$ for subespaço. Os exemplos 1.2 .2 e 5.4.5 mostram que pode acontecer $Q_{C}(x) \neq R_{C}(x)$.

A seguir veremos certas condições em que $Q_{C}$ e $R_{C}$ são iguais.

Teorema 5.4.10. Se para todo $c_{0} \in R_{C}(x)$ temos que $c_{0} \in R_{C}\left(c_{0}+t\left(x-c_{0}\right)\right)$, para $0 \leq t \leq 1$, então

$$
R_{C}(x)=Q_{C}(x)
$$

\section{Demonstração :}

Pelo teorema 5.4.6, só precisamos provar que $R_{C}(x) \subset Q_{C}(x)$, ou seja, que para todo $c_{0} \in R_{C}(x)$, temos $\tau\left(c_{0}-c, x-c_{0}\right) \geq 0$, para todo $c \in C$.

Como $\tau\left(c_{0}-c, x-c_{0}\right)=\lim _{t \rightarrow 0^{+}} \frac{\left\|c_{0}-c+t\left(x-c_{0}\right)\right\|-\left\|c_{0}-c\right\|}{t}$, existe sempre (observação 5.4.2), basta provar que para todo $0<t,\left\|c_{0}-c+t\left(x-c_{0}\right)\right\| \geq\left\|c_{0}-c\right\|$, para todo $c \in C$. Mas isso é fácil deduzir, pois, como $c_{0} \in R_{C}\left(c_{0}+t\left(x-c_{0}\right)\right)$, temos que para todo $0 \leq t \leq 1$ e todo $c \in C$ vale

$$
\left\|c_{0}-c\right\| \leq\left\|c_{0}+t\left(x-c_{0}\right)-c\right\|=\left\|c_{0}-c+t\left(x-c_{0}\right)\right\| .
$$


Observação 5.4.11. De fato. no teorema anterior, não é preciso que $0 \leq t \leq 1$. Basta que exista $\alpha>0$ tal que para $0 \leq t \leq \alpha$ tenhamos $c_{0} \in R_{C}\left(c_{0}+t\left(x-c_{0}\right)\right)$.

Corolário 5.4.12. $c_{0} \in Q_{C}(x)$ se e somente se, $c_{0} \in R_{C}\left(c_{0}+t\left(x-c_{0}\right)\right)$ para todo $t \geq 0$.

\section{Prova :}

É conseqüência imediata dos teoremas 5.4 .6 e 5.4.10 e da proposição 5.3.2.

Exemplo 5.4.13. Sejam $X=\left(\mathbb{R}^{3},\|\|_{\infty}\right), C=\left\{\left(x_{1}, x_{2}, 0\right) \in \mathbb{R}^{3}:\left|x_{2}\right| \leq 2\right\}$ e $x=(0,0,1)$. Resulta simples ver que $R_{C}(x)=\{(0,0,0)\}$, pois $C \subset H$, onde $H$ é o plano $x_{3}=0$ e $R_{H}(x)=\{(0,0,0)\}$. Portanto, $(0,0,0) \in R_{C}(x)$. Como se observa na figura $5.4,(0,0,0)$ é o único ponto que pertence a $R_{C}(x)$. Isso acontece porque

$$
\bigcap_{i=1}^{i=4}\left(\bar{B}_{2}\left(c_{i}\right)\right)=\left(\bigcap_{i=1}^{i=2}\left(\bar{B}_{2}\left(c_{i}\right)\right)\right) \cap\left(\bigcap_{i=3}^{i=4}\left(\bar{B}_{2}\left(c_{i}\right)\right)\right)=S_{1} \cap S_{2}=\{(0,0,0)\} .
$$

Também temos que $R_{C}(y)=\{(0,0,0)\}$ para qualquer $y=(0,0, t)$ com $0 \leq t \leq 1$. Portanto, pelo teorema 5.4.10, $Q_{C}(x)=R_{C}(x)$ e então $Q_{C}(x)=\{(0,0,0)\}$.

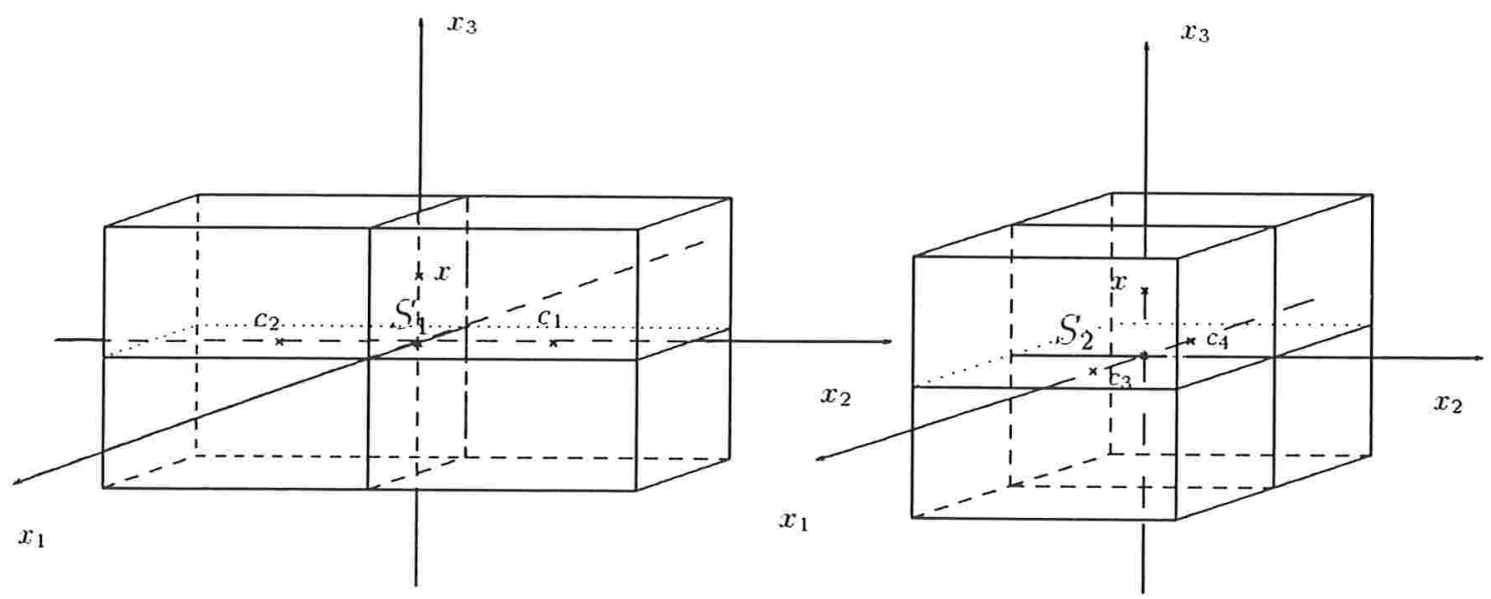

Figura 5.4.

Teorema 5.4.14. Se para quaisquer $c \in C$ e $c_{0} \in R_{C}(x)$, temos que $(1-t) c_{0}+t c \in C$, para todo $t \geq 1$, então

$$
R_{C}(x)=Q_{C}(x)
$$




\section{Demonstração :}

Novamente, pelo teorema 5.4.6, só precisamos provar que $R_{C}(x) \subset Q_{C}(x)$.

Como $c_{0} \in R_{C}(x)$, entào para todo $c \in C$

$$
\left\|c_{0}-c\right\| \leq\|c-x\| .
$$

Em particular, se $t \geq 1$, essa desigualdade vale para $c_{0}+t\left(c-c_{0}\right)$, que por hipótese é um elemento de $C$. Logo,

$$
\left\|t\left(c_{0}-c\right)\right\| \leq\left\|c_{0}+t\left(c-c_{0}\right)-x\right\|=\left\|x-c_{0}+t\left(c_{0}-c\right)\right\| .
$$

Dividindo por $t$ e tomando $s=\frac{1}{t}$ a expressão fica

$$
\left\|c_{0}-c\right\| \leq\left\|s\left(x-c_{0}\right)+c_{0}-c\right\| .
$$

Portanto,

$$
\left\|s\left(x-c_{0}\right)+c_{0}-c\right\|-\left\|c_{0}-c\right\| \geq 0, \text { para qualquer } 0 \leq s \leq 1 .
$$

Assim,

$$
\tau\left(c_{0}-c, x-c_{0}\right)=\lim _{s \rightarrow 0^{+}} \frac{\left\|c_{0}-c+s\left(x-c_{0}\right)\right\|-\left\|c_{0}-c\right\|}{s} \geq 0
$$

ou seja,

$$
c_{0} \in Q_{C}(x) .
$$

Observação 5.4.15. Na verdade, toda a semi-reta com início em $c_{0}$ passando por $c$ está contida em $C$, pois, pela convexidade de $C, c_{0}+t\left(c-c_{0}\right) \in C$ para $0 \leq t \leq 1$.

Exemplo 5.4.16. Seja $X=\left(\mathbb{R}^{2},\|\|\right)$, onde a norma \|\| é dada da seguinte maneira:

$$
\|(a, b)\|=\left\{\begin{array}{cl}
\frac{|b|}{3}, & \text { se } a=0 \\
\frac{|a|}{2}, & \text { se } b=0 \\
\frac{|a|}{2}-\frac{|b|}{3}, & \text { se }-\frac{a}{b} \geq 1, b \neq 0 \\
\frac{|a|}{2}+\frac{|b|}{3}, & \text { se }-\frac{a}{b} \leq 0, b \neq 0 \\
-\frac{|a|}{2}+\frac{|b|}{3}+\frac{|a|^{3}}{3|b|^{2}}, & \text { se } 0<-\frac{a}{b}<1, b \neq 0 .
\end{array}\right.
$$


Na figura 5.5, temos um esquema para interpretar melhor essa norma e na figura 5.6 temos uma representação da bola unitária de $X$.

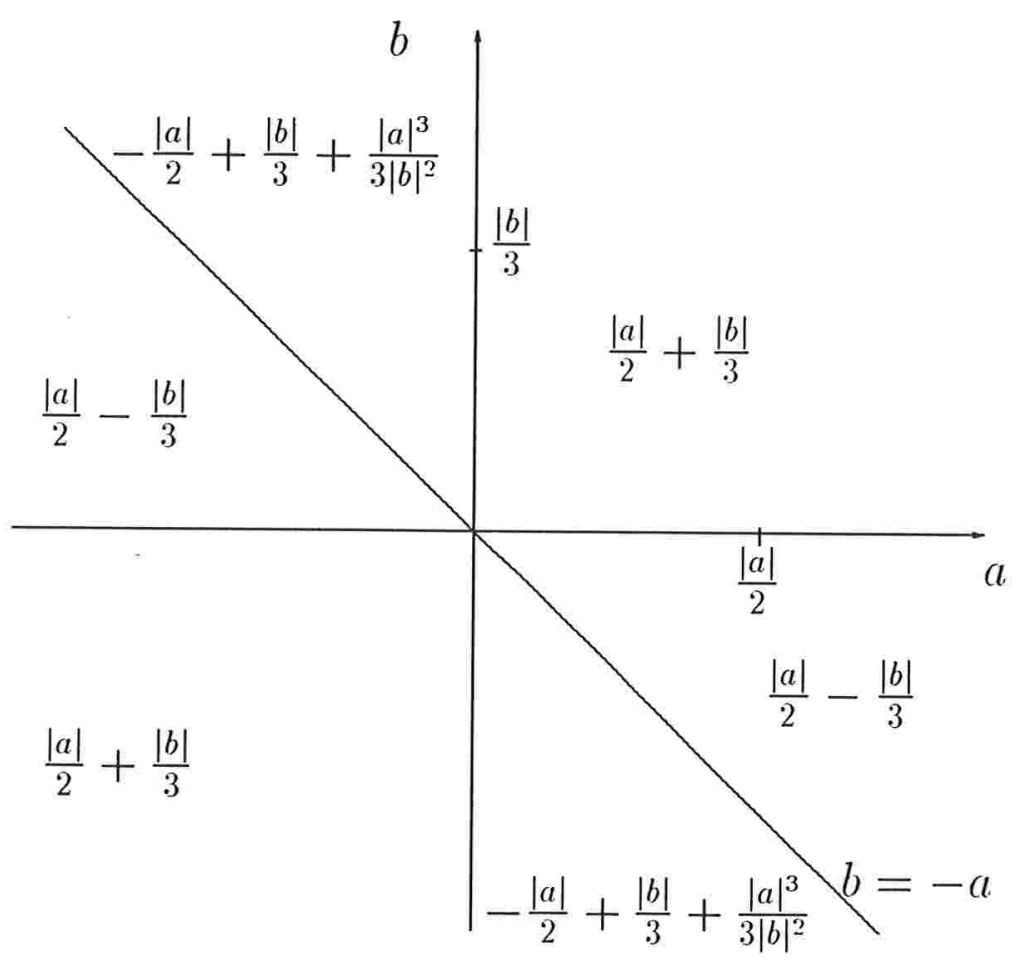

Figura 5.5. 


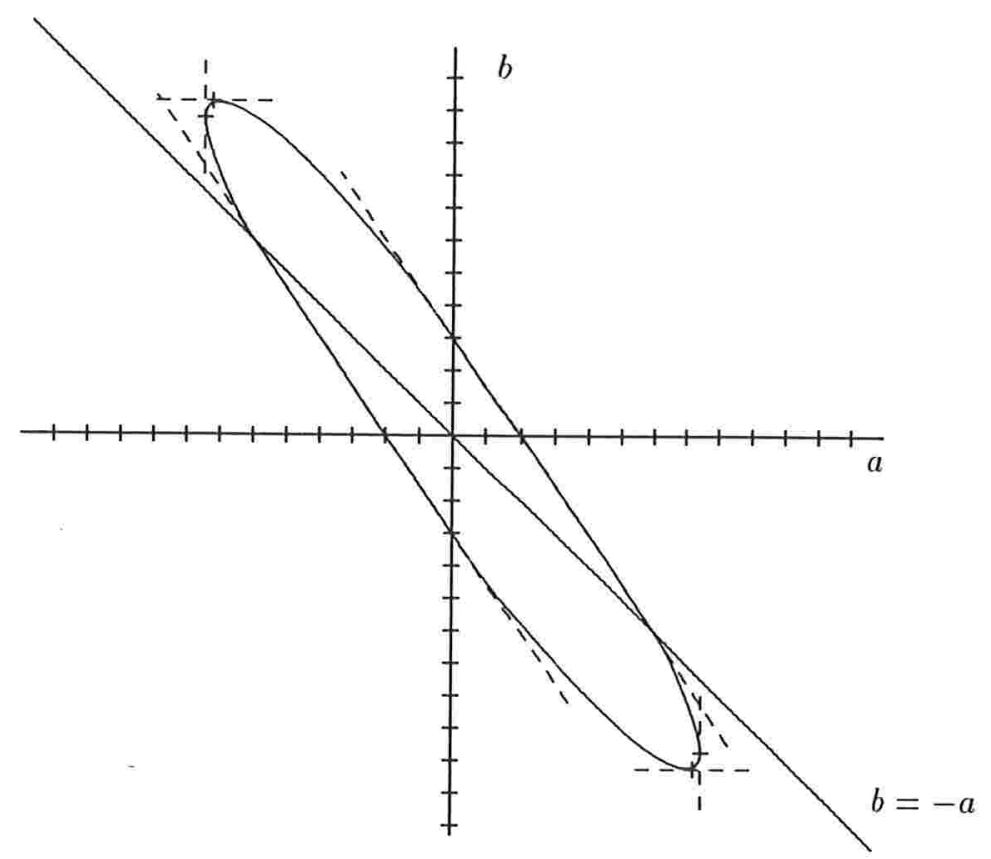

Figura 5.6.

Consideremos também $C=\left\{(a, b) \in \mathbb{R}^{2}: a \geq 1\right\}$ e $x=(0,0)$. Com essas condições deduzimos que

$$
R_{C^{\prime}}(x)=\left\{\left(1,-\frac{3}{2}\right)\right\} .
$$

Provamos isso completamente, mas as deduçòes não estão incluidas neste trabalho. Daremos, na figura 5.7 uma interpretação geométrica. 


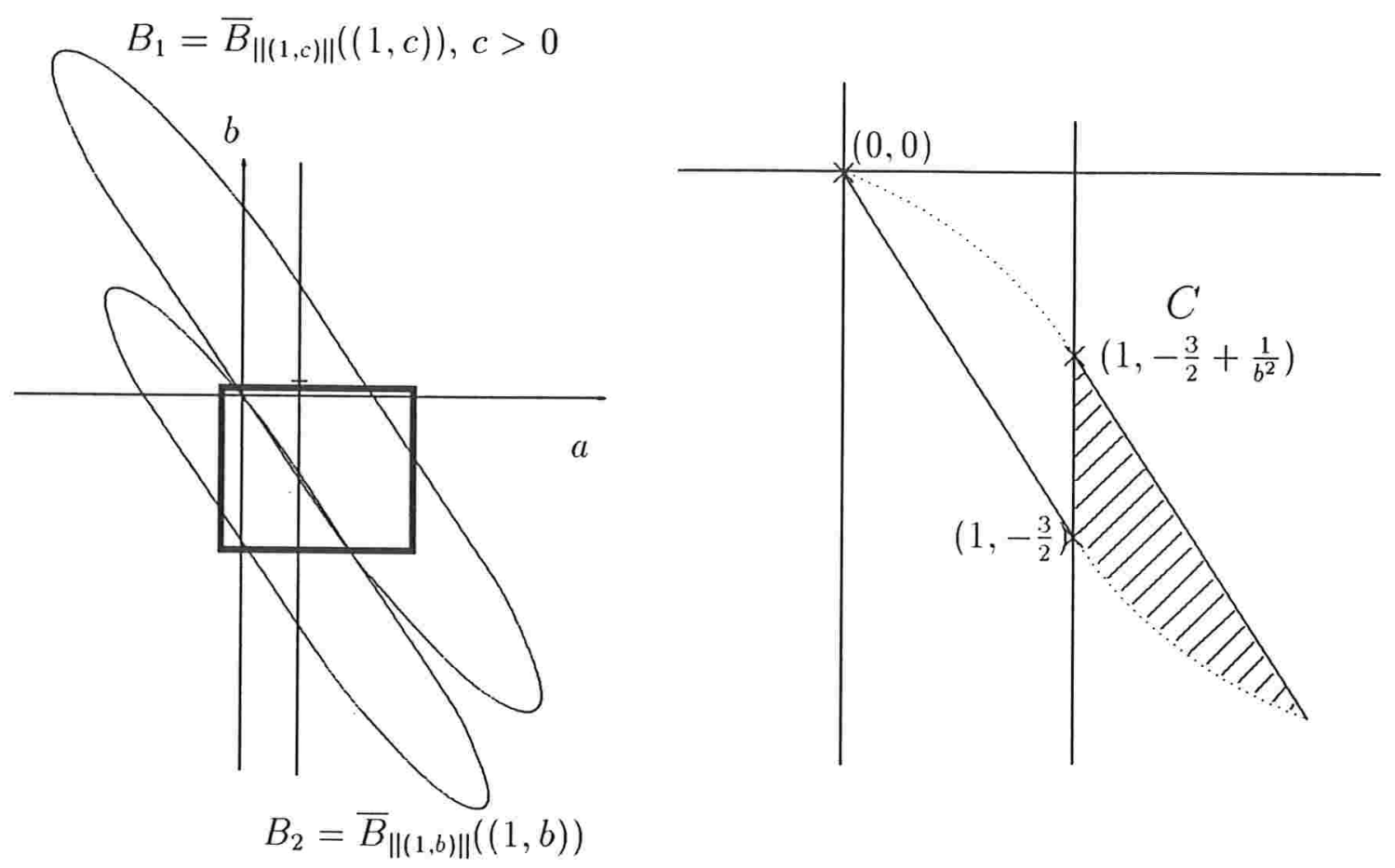

\section{Figura 5.7.}

Na figura, a região onde se interseptam $B_{1}$ e $B_{2}$, foi ampliada. Resulta bastante simples concluir que no caso de se tomar o limite quando $b \rightarrow-\infty$, o único ponto que permanecerá na intersecção de $B_{1}$ e $B_{2}$ é efetivamente $\left(1,-\frac{3}{2}\right)$.

Observamos que $C$ não é subespaço mas satisfaz as condições do teorema 5.4.14 e portanto também sabemos que

$$
Q_{C}(x)=\left\{\left(1,-\frac{3}{2}\right)\right\}
$$




\subsection{Aproximação, coaproximação e retração orto- gonal fortes}

Nesta seção estudaremos subconjuntos de $P_{C}, R_{C}$ e $Q_{C}$ especiais, cujos elementos chamaremos de aproximações fortes.

Definição 5.5.1. Seja $x \in X \backslash C$. Dizemos que $c_{0}$ pertence fortemente a $P_{C}(x)$ se existir $r>0$ tal que

$$
\|x-c\| \geq\left\|x-c_{0}\right\|+r\left\|c_{0}-c\right\| \text { para todo } c \in C \text {. }
$$

O conjunto dos elementos que satisfazem esta definição será representado por $P_{C}^{F}(x)$.

Exemplo 5.5.2. Sejam $X=\left(\mathbb{R}^{2},\|\|_{2}\right), C$ o eixo $x_{1}$ e $x=(0,1)$. É claro que $P_{C}(x)=\{(0,0)\}$. Veremos que $(0,0) \notin P_{C}^{F}(x)$.

vale

Para $(0,0)$ pertencer a $P_{C}^{F}(x)$. deve existir $r>0$ tal que para todo $t \in \mathbb{R}$

$$
\|(0,1)-(t, 0)\| \geq r\|(0,0)-(t, 0)\|+\|(0,1)-(0,0)\|,
$$

ou seja,

$$
\sqrt{1+t^{2}} \geq r|t|+1, \quad \text { para todo } t \in \mathbb{R}
$$

o que não é verdade. (figura 5.8)

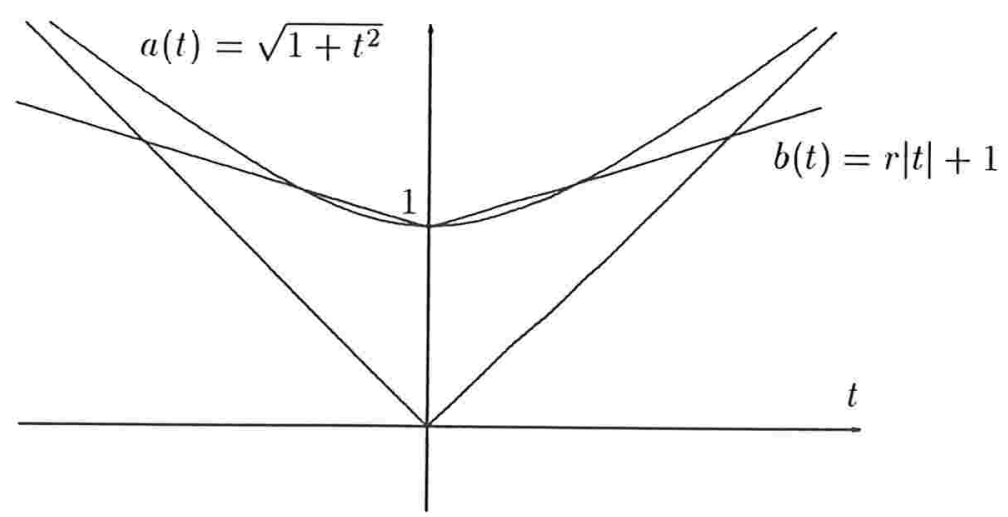

Figura 5.8. 
Exemplo 5.5.3. Seja $X=\left(\mathbb{R}^{2},\|\|_{2}\right), C=\left\{\left(x_{1}, x_{2}\right) \in \mathbb{R} \times \mathbb{R}:-x_{2} \geq\left|x_{1}\right|\right\} \mathrm{e}$ $x=(0,1)$. Claramente $P_{C}(x)=\{(0,0)\}=\left\{c_{0}\right\}$. Veremos que $c_{0} \in P_{C:}^{F}(x)$, ou seja, que existe $r>0$ tal que

$$
\|x-c\| \geq\left\|x-c_{0}\right\|+r\left\|c_{0}-c\right\| \text { para todo } c \in C .
$$

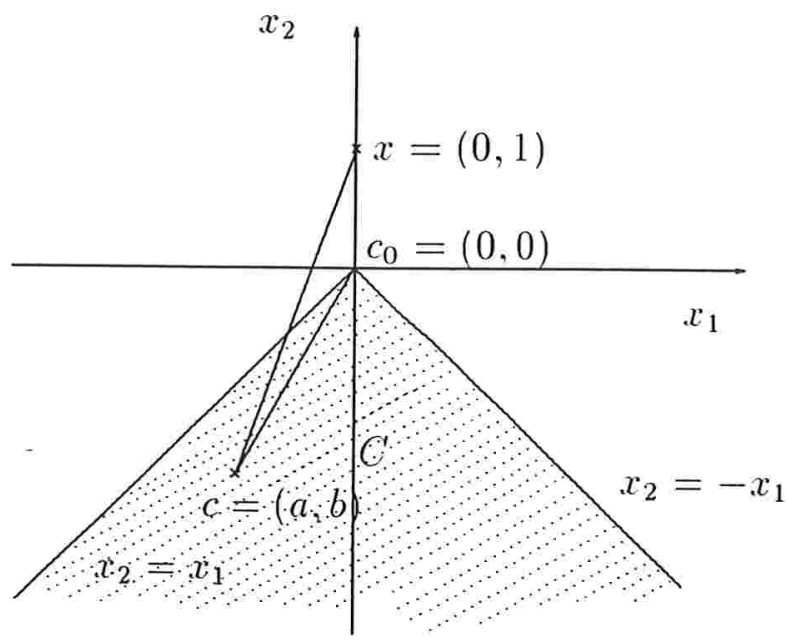

Figura 5.9. O ponto $(0,0) \in P_{C}^{F}(x)$.

Se $c=(0,0)$, então qualquer $r$ satisfaz a desigualdade. Seja então $c \in C \backslash\{(0,0)\}$, $c=(a, b)$.

Trabalhando em função dos parâmetros $a$ e $b$. a desigualdade anterior fica

$$
\sqrt{a^{2}+(b-1)^{2}} \geq 1+r \sqrt{a^{2}+b^{2}} .
$$

Consideremos $f: C \backslash\{(0,0)\} \rightarrow \mathbb{R}$ dada por $f(a \cdot b)=\frac{\sqrt{a^{2}+(b-1)^{2}}-1}{\sqrt{a^{2}+b^{2}}}$. Estudemos $f$ em $B_{\varepsilon}((0,0))$. Temos, para $\varepsilon$ suficientemente pequeno

$$
\begin{gathered}
f(a, b)=\frac{a^{2}+b^{2}-2 b}{\sqrt{a^{2}+b^{2}}\left(\sqrt{a^{2}+(b-1)^{2}}+1\right)} \geq \frac{a^{2}+b^{2}-2 b}{4 \sqrt{a^{2}+b^{2}}} \geq \\
\geq \frac{-b}{2 \sqrt{a^{2}+b^{2}}} \geq \frac{-b}{2 \sqrt{2 b^{2}}}=\frac{1}{2 \sqrt{2}} .
\end{gathered}
$$

Logo, em $C_{1}=B_{\varepsilon}(0) \cap C$, vale $f(a, b) \geq \frac{1}{2 \sqrt{2}}$. 
Vamos ver se existe $k>0$ tal que $f \geq k$ para pontos fora de $B_{\varepsilon}((0,0))$.

Continuando com o estudo da $f$, temos

$$
\begin{gathered}
f(a, b)=\frac{a^{2}+b^{2}-2 b}{\sqrt{a^{2}+b^{2}}\left(\sqrt{a^{2}+(b-1)^{2}}+1\right)} \geq \frac{a^{2}+b^{2}-2 b}{\sqrt{a^{2}+b^{2}}\left(\sqrt{b^{2}+(b-1)^{2}}+1\right)} \geq \\
\geq \frac{b^{2}(1-2 / b)}{\sqrt{2 b^{2}}\left(\sqrt{b^{2}\left(1+\left(\frac{b-1}{b}\right)^{2}\right)}+1\right)}=\frac{1-2 / b}{\sqrt{2}\left(\sqrt{1+\left(\frac{b-1}{b}\right)^{2}}-1 / b\right)} \rightarrow \frac{1}{2} \text { quando } b \rightarrow-\infty .
\end{gathered}
$$

Portanto, podemos afirmar que para algum $k_{0} \in \mathbb{R}^{+}$, vale $f(a, b) \geq \frac{1}{2 \sqrt{2}}$ para todo $c=(a, b) \in C_{2}=\left\{\left(x_{1}, x_{2}\right):\left\|\left(x_{1}, x_{2}\right)\right\|>k_{0}\right\}$.

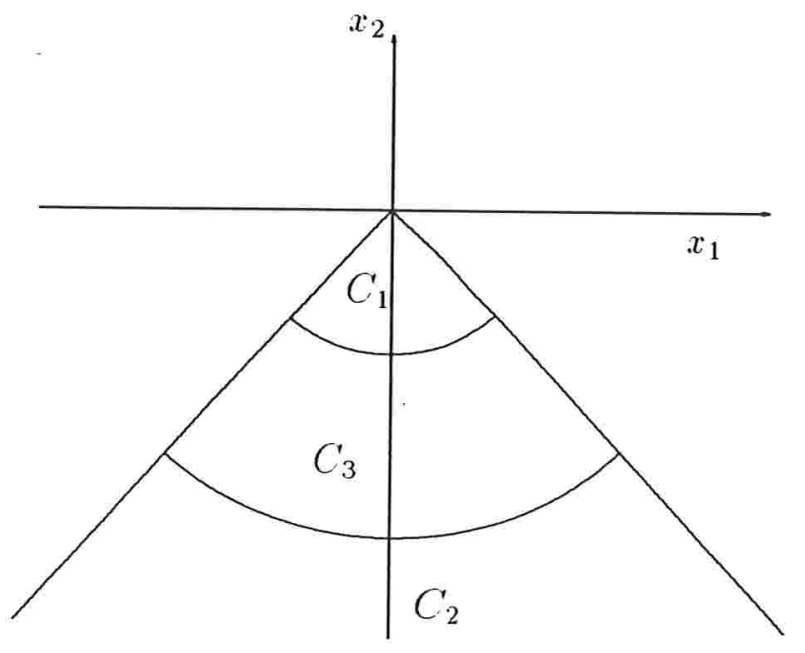

Figura 5.10.

Se $k_{0}<\varepsilon$, temos que $r=\frac{1}{2 \sqrt{2}}$ satisfaz (5.4) para todo $c \in C$. Caso contrário, resta estudar $f$ na região $C_{3}=\left\{\left(x_{1}, x_{2}\right): \varepsilon \leq\left\|\left(x_{1}, x_{2}\right)\right\| \leq k_{0}\right\}$. Como $C_{3}$ é compacto e $f$ é contínua, então existe $\left(a_{0}, b_{0}\right) \in C_{3}$ tal que $f$ atinge seu minimo nele. Seja $h$ esse valor mínimo. Também temos que tal mínimo é estritamente positivo pois $f$ o é.

Tomando

$$
r=\min \left\{h, \frac{1}{2 \sqrt{2}}\right\},
$$

temos que $r$ satisfaz as condiçòes de (๖.4). 
Exemplo 5.5.4. Sejam $\mathrm{X}=\left(\mathbb{R}^{2},\|\|_{2}\right), C=\left\{\left(x_{1}, x_{2}\right) \in \mathbb{R}^{2}: x_{1}^{2}+x_{2}^{2} \leq 1\right\} \mathrm{e}$ $x=(2,0)$. Neste caso $P_{C}(x)=\{(1,0)\}$, mas esse ponto não pertence fortemente ao conjunto.

Se $c=(1,0)=c_{0}$, então qualquer $r$ satisfaz

$$
\|x-c\| \geq\left\|x-c_{0}\right\|+r\left\|c_{0}-c\right\| .
$$

Tomemos $c=(a, b) \in C \backslash\left\{c_{0}\right\}$ tal que $a^{2}+b^{2}=1$. A desigualdade (5.5) fica

$$
\sqrt{-4 a+5} \geq 1+r \sqrt{-2 a+2} .
$$

Provaremos que não existe $r>0$ tal que

$$
\frac{\sqrt{-4 a+5}-1}{\sqrt{-2 a+2}} \geq r, \text { para todo }-1 \leq a<1 \text {. }
$$

Fazendo algumas operações, obtemos que

$$
\frac{\sqrt{-4 a+5}-1}{\sqrt{-2 a+2}}=\frac{2 \sqrt{2} \sqrt{-a+1}}{\sqrt{-4 a+5}+1} \rightarrow 0 \text { quando } a \rightarrow 1^{-} .
$$

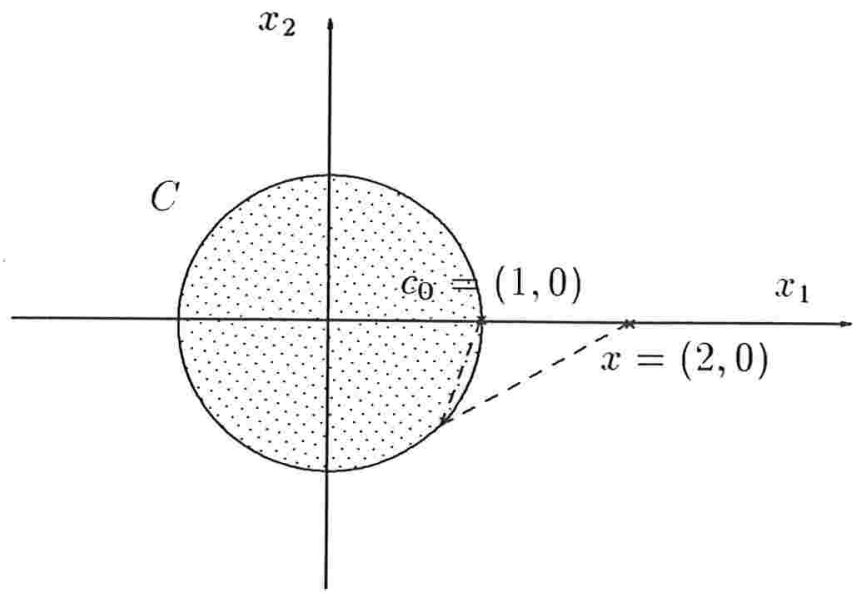

Figura 5.11. O ponto $(1,0) \notin P_{C}^{F}(x)$.

Definição 5.5.5. Seja $x \in X \backslash C$. Dizemos que $c_{0}$ pertence fortemente a $R_{C}(x)$ se existir $r>0$ tal que

$$
\|x-c\| \geq\left\|c_{0}-c\right\|+r\left\|c_{0}-x\right\| \text { para todo } c \in C .
$$

$\mathrm{O}$ conjunto dos elementos que satisfazem esta definição será representado por $R_{C}^{F}(x)$. 
Observação 5.5.6. A desigualdade (5.6) pode ser escrita como

$$
\|x-c\| \geq\left\|c_{0}-c\right\|+k \text { para todo } c \in C,
$$

onde $k>0$ qualquer, porque, de fato, $\left\|c_{0}-x\right\| \neq 0$ e não depende de $c$.

Exemplo 5.5.7. Sejam $X=\left(\mathbb{R}^{2},\|\|_{2}\right), C=\left\{\left(x_{1}, x_{2}\right) \in \mathbb{R}^{2}: x_{1}^{2}+x_{2}^{2} \leq 1\right\}$ e $x=$ $(3,0)$. Lembramos, do exemplo 1.2.2, que $R_{C}(x)=C$. Veremos que $c_{0} \in C \backslash\{(-1,0)\}$ pertence fortemente a $R_{C}(x)$ e que $(-1,0) \notin R_{C}^{F}(x)$.

Primeiro estudemos $(-1,0)$. Tomando $c=(1,0)$ temos que $\|x-c\|=2=\left\|c_{0}-c\right\|$, o que significa que $(-1,0)$ nào pertence fortemente a $R_{C}(x)$.

Seja agora $c_{0} \in C \backslash\{(-1,0)\}$. Devemos provar que existe $k>0$ tal que $\|x-c\| \geq$ $\left\|c_{0}-c\right\|+k$ para todo $c \in C$.

Tomando $c \in C$ e utilizando as definições de distância e diâmetro,

$$
\|x-c\| \geq \operatorname{dist}(x, C)=2=\operatorname{diam}(C) \geq\left\|c_{0}-c\right\| .
$$

Logo, $\|x-c\|-\left\|c_{0}-c\right\| \geq 0$. Definamos $k=\inf _{c \in C}\left\{\|x-c\|-\left\|c_{0}-c\right\|\right\}$ e suponhamos que $k=0$. Como $C$ é compacto e a norma é uma função contínua, temos que existe $c_{1} \in C$ tal que $\left\|x-c_{1}\right\|-\left\|c_{0}-c_{1}\right\|=0$ e portanto

$$
\left\|x-c_{1}\right\|=2=\left\|c_{0}-c_{1}\right\| .
$$

Daí, $c_{1}=(1,0)$ e $c_{0}=(-1,0)$, o que não é verdade.

Definição 5.5.8. Seja $x \in X \backslash C$. Dizemos que $c_{0}$ pertence fortemente a $Q_{C}(x)$ se existir $r>0$ tal que

$$
\tau\left(c_{0}-c, x-c_{0}\right) \geq r\left\|x-c_{0}\right\| \text { para todo } c \in C .
$$

O conjunto dos elementos que satisfazem esta definição será representado por $Q_{C}^{F}(x)$.

Exemplo 5.5.9. Sejam $\mathrm{X}=\left(\mathbb{R}^{2},\|\|_{2}\right), C=\left\{\left(x_{1}, x_{2}\right) \in \mathbb{R}^{2}: x_{1}^{2}+x_{2}^{2} \leq 1\right\} \mathrm{e}$ $x=(3,0)$. Veremos que $c_{0}=(-1,0)$ não pertence fortemente a $Q_{C}(x)$. 
Como vimos no exemplo 5.4.5, se $c=(a, b) \in C$,

$$
\tau\left(c_{0}-c, x-c_{0}\right)=\frac{1-a}{2 \sqrt{a^{2}+b^{2}-2 a+1}} .
$$

Tomando $c=(a, b)$ tal que $a^{2}+b^{2}=1$, temos

$$
\tau\left(c_{0}-c, x-c_{0}\right)=\frac{1-a}{2 \sqrt{2(1-a)}}=\frac{\sqrt{1-a}}{2 \sqrt{2}} \rightarrow 0 \text { quando } a \rightarrow 1^{-} .
$$

Portanto, $c_{0} \notin Q_{C}^{F}(x)$.

Exemplo 5.5.10. Sejam $X=\left(\mathbb{R}^{2},\|\|_{2}\right), C$ o eixo dos $x_{1}$ e $x=(0,1)$. Tomemos $c=(a, 0) \in C$. Veremos que $c_{0}=(0,0) \notin Q_{C}^{F}(x)$.

Fazendo operações simples, obtemos que

$$
\tau\left(c_{0}-c, x-c_{0}\right)=\lim _{t \rightarrow 0^{+}} \frac{\left\|c_{0}-c+t\left(x-c_{0}\right)\right\|-\left\|c_{0}-c\right\|}{t}=0 .
$$

Logo, $c_{0} \notin Q_{C}^{F}(x)$.

Exemplo 5.5.11. Consideremos novamente as condições de exemplo 5.5.3, ou seja, $X=\left(\mathbb{R}^{2},\|\|_{2}\right), C=\left\{\left(x_{1}, x_{2}\right) \in \mathbb{R} \times \mathbb{R}^{-}:-x_{2} \geq\left|x_{1}\right|\right\}$ e $x=(0,1)$. Veremos que $c_{0}=(0,0) \in Q_{C}^{F}(x)$.

Observemos que se $c=c_{0}$, qualquer $0 \leq r \leq 1$ serve. Portanto, podemos supor $c \neq c_{0}, c=(a, b)$. Então

$$
\begin{gathered}
\lim _{t \rightarrow 0^{+}} \frac{\left\|c_{0}-c+t\left(x-c_{0}\right)\right\|-\left\|c_{0}-c\right\|}{t}=\lim _{t \rightarrow 0^{+}} \frac{\|(-a, t-b)\|^{2}-\|(a, b)\|^{2}}{t\left(\left\|c_{0}-c+t\left(x-c_{0}\right)\right\|+\left\|c_{0}-c\right\|\right)}= \\
\quad=\lim _{t \rightarrow 0^{+}} \frac{t(t-2 b)}{t\left(\left\|c_{0}-c+t\left(x-c_{0}\right)\right\|+\left\|c_{0}-c\right\|\right)}=\frac{|b|}{\sqrt{a^{2}+b^{2}}} \geq \frac{|b|}{\sqrt{2 b^{2}}}=\frac{1}{\sqrt{2}} .
\end{gathered}
$$

Logo, $c_{0}=(0,0) \in Q_{C}^{F}(x)$. 
Teorema 5.5.12. $Q_{C}^{F}(x) \subset R_{C}^{F}(x)$.

Demonstração :

Seja $c_{0} \in Q_{C}^{F}(x)$. Sabemos que existe $r>0$ tal que para todo $c \in C$, vale

$$
\tau\left(c_{0}-c, x-c_{0}\right)=\lim _{t \rightarrow 0^{+}} \frac{\left\|c_{0}-c+t\left(x-c_{0}\right)\right\|-\left\|c_{0}-c\right\|}{t} \geq r\left\|x-c_{0}\right\| .
$$

Pela proposição 2.3.4, tomando $t=1$ obtemos para todo $c \in C$

$$
\|x-c\|-\left\|c_{0}-c\right\| \geq r\left\|x-c_{0}\right\| .
$$

Teorema 5.5.13. Se para quaisquer $c \in C$ e $c_{0} \in R_{C}(x)$, temos que $c_{0}+t\left(c-c_{0}\right) \in C$, então, se $c_{0} \in R_{C}^{F}(x)$, também $c_{0} \in Q_{C}^{F}(x)$.

\section{Demonstração :}

Provaremos que existe $r>0$ tal que $\tau\left(c_{0}-c, x-c_{0}\right) \geq r\left\|x-c_{0}\right\|$, para todo $c \in C$.

Temos

$$
\begin{gathered}
\tau\left(c_{0}-c, x-c_{0}\right)=\lim _{s \rightarrow 0^{+}} \frac{\left.\left\|c_{0}-c+s\left(x-c_{0}\right)\right\|-\left\|c_{0}-c\right\|\right)}{s}= \\
=\lim _{t \rightarrow+\infty}\left\|t\left(c_{0}-c\right)+x-c_{0}\right\|-\left\|t\left(c_{0}-c\right)\right\|= \\
=\lim _{t \rightarrow+\infty}\left\|x-\left(c_{0}+t\left(c_{0}-c\right)\right)\right\|-\left\|c_{0}-\left(c_{0}+t\left(c_{0}-c\right)\right)\right\| .
\end{gathered}
$$

Como $c_{0}+t\left(c-c_{0}\right) \in C$ e $c_{0} \in R_{C}^{F}(x)$, temos que existe $r>0$ com $\left\|x-\left(c_{0}+t\left(c_{0}-c\right)\right)\right\|-\left\|c_{0}-\left(c_{0}+t\left(c_{0}-c\right)\right)\right\| \geq r\left\|x-c_{0}\right\|$ para todo $t \geq 1$ e todo $c \in C$.

Daí,

$$
\tau\left(c_{0}-c, x-c_{0}\right) \geq r\left\|x-c_{0}\right\|
$$


Corolário 5.5.14. Se C for um subespaço. então $R_{C}^{F}(x)=Q_{C}^{F}(x)$.

\section{Demonstração :}

Imediata.

Observação 5.5.15. No caso de $C$ ser subespaço, temos que $c_{0} \in R_{C}^{F}(x)$ se e só se existe $r>0$ com

$$
\tau\left(m, x-c_{0}\right) \geq r\left\|x-c_{0}\right\| \text { para todo } m \in C \backslash\{0\} .
$$

Teorema 5.5.16. Seja $A_{r}=\left\{c_{0} \in R_{C}(x):\left\|c_{0}-c\right\|+r\left\|c_{0}-x\right\| \leq\|x-c\|, c \in C\right\}$, com $r>0$. Então $r\left(A_{r}\right) \leq(1-r) d$, onde $d=\operatorname{dist}(x, C) \in r\left(A_{r}\right)$ é o raio de Chebyshev de $A_{r}$.

\section{Demonstração :}

Lembramos a definição de raio de Chebyshev de um conjunto $A$,

$$
r(A)=\inf _{x \in X}\left[\sup _{c \in A}\|x-c\|\right]
$$

e que claramente $r(A) \leq \sup _{c \in A}\|x-c\|$ para todo $x \in X$.

Tomemos, para cada $\varepsilon>0, c_{\varepsilon} \in C$ tal que $\left\|x-c_{\varepsilon}\right\|<d+\varepsilon$.

Agora, tomando $c_{0} \in A_{r}$, também temos que

$$
\left\|c_{0}-c_{\varepsilon}\right\| \leq\left\|x-c_{\varepsilon}\right\|-r\left\|c_{0}-x\right\|<d+\varepsilon-r d=(1-r) d+\varepsilon .
$$

Por $\left({ }^{*}\right)$,

$$
r\left(A_{r}\right) \leq \sup _{c \in A_{r}}\left\|c-c_{\varepsilon}\right\| \leq(1+r) d+\varepsilon .
$$

Como $\varepsilon$ é arbitrário, concluimos

$$
r\left(A_{r}\right) \leq(1-r) d
$$

Teorema 5.5.17. Seja C um subespaço. Se $x$ tem melhor coaproximação forte em $C$, então todo elemento de $[\{x\} \cup C]$ também tem. Mais precisamente, se $c_{0} \in R_{C}^{F}(x)$, então para quaisquer $k \in \mathbb{R}$ e $c_{1} \in C$. temos $k c_{0}+c_{1} \in R_{C}^{F}\left(k x+c_{1}\right)$ e com o mesmo $r$. 


\section{Demonstração :}

Como $C$ é subespaço, por 5.5.14, provaremos que $k c_{0}+c_{1} \in Q_{C}^{F}\left(k x+c_{1}\right)$ para quaisquer $k \in \mathbb{R}$ e $c_{1} \in C$. Como para $k=0$ é imediato, consideraremos $k \neq 0$. Vamos usar 5.5.15. Seja $m \in C \backslash\{0\}$. Então

$$
\begin{gathered}
\tau\left(m, k x+c_{1}-\left(k c_{0}+c_{1}\right)\right)=\tau\left(m, k\left(x-c_{0}\right)\right)= \\
=\lim _{t \rightarrow 0^{+}} \frac{\left\|m+t k\left(x-c_{0}\right)\right\|-\left\|k\left(x-c_{0}\right)\right\|}{t}=\lim _{t \rightarrow 0^{+}}|k| \frac{\left\|\frac{m}{k}+t\left(x-c_{0}\right)\right\|-\left\|\left(x-c_{0}\right)\right\|}{t}= \\
=|k| \tau\left(\frac{m}{k}, x-c_{0}\right) \geq|k| r\left\|x-c_{0}\right\|=r\left\|k x+c_{1}-\left(k c_{0}+c_{1}\right)\right\| .
\end{gathered}
$$

Logo,

$\tau\left(m, k x+c-\left(k c_{0}+c\right)\right) \geq r\left\|k x+c-\left(k c_{0}+c\right)\right\|$ para todo $m \in C \backslash\{0\}$.

Observação 5.5.18. O exemplo 5.4.16 nos dá um exemplo de $C$ não subespaço com $R_{C}^{F}=Q_{C}^{F}$. 


\section{Referências Bibliográficas}

[B-M73] M.W.Bartelt \& H.W.McLaughlin. Characterizations of strong unicity in approximation theory, J. Approx. Theory 9, (1973), 255-266.

[Bea82] B.Beauzamy. Introduction to Banach spaces and their geometry, NorthHoland Math. Studies 68, (1982).

[Bel68] P.K.Belobrov. Some criteria for the Hilbertness of a space, Izv. Vysš. Ucčbn. Zaved. Matematika, 9 (76) (1968), 13-15 (russian).

[Bir35] G.Birkhoff. Orthogonality in linear spaces, Duke Math. Jour. vol. 1 (1935), 169-172.

[Bru73] R.E.Bruck Jr. Nonexpansive projections on subsets of Banach spaces, Pacific J. Math. 47 (1973), 341-355.

[Con82] J.B.Conway. A course in funtional analysis, Springer-Verlag, New York, (1970).

[Dav63] P.Davis. Interpolation and approximation, New York, Blaisdell, (1963).

[D-S58] N.Durford \& J.T.Schwartz. Linear operators, New York, Interscience P. Jhon Wiley, (1988).

[Fra75] C.Franchetti. Chebyshev centers and hipercycles, Boll. Un. Mat. Ital.(4) 47 (1975), 565-573.

[F-F72] C.Franchetti \& M. Furi. Some characteristic properties of real Hilbert spaces, Rev. Roumaine Math. Pures Appl. 17 (1972), 1045-1048.

[Gil65] J.R.Giles. Classes of semi-inner-product spaces, Trans. Amer. Math. Soc. 129 (1945), 436-446.

[Hol72] R.B.Holmes. A course on optimization and best approximation, SpringerVerlag, New York (1972). 
[Jam45] R.C.James. Orthogonality in normed linear spaces, Duke Math. J. 12 (1945), 291-302.

[Jam47a] R.C.James. Orthogonality and linear functionals in normed linear spaces, Trans. Amer. Math. Soc. 61 (1947), 265-292.

[Jam47b] R.C..James. Inner products in normed linear spaces, Bull. Amer. Math. Soc. 53 (1947), 559-566.

[Kak39] S.Kakutani. Some characterizations of Euclidean spaces, Jap. J. Math. 16 (1939), 93-97.

[Nar91] T.D.Narang. On best coapproximation in normed linear spaces, Rocky Moun. J. Math. 22 (1991), 265-28i.

[MutS0] S.Muthukumar. A note on best simultanєous aproximations, Indian J. Pure Appl. Math. 11 (1980), 715-719.

[Pap74] P.L.Papini. Some questions related to the concept of orthogonality in Banach spaces. Orthogonal projetions, Boll. Un. Mat. Ital. (4) 9 (1974), 386-401.

[Pap75] P.L.Papini. Some questions related to the concept of orthogonality in Banach spaces, proximity map; bases, Boll. Un. Mat. Ital. (4) 11 (1975), 44-63.

[Pap76] P.L.Papini. Approximation and strong approximation in normed spaces via tangent functionals, J. Approx. Theory 22 (1976), 111-118.

[P-S79] P.L.Papini \& I. Singer. Best coapproximation in normed linear spaces, Monatsh. Math. 88 (1979), 27-44.

[Phe89] R.R.Pheps. Convex functions, monotone operators and differenciability, Berlin, Springer-Verlag (1989).

[Sin70] I.Singer. Best approximation in normed linear spaces by elements of linear subespaces, Springer-Verlag, New York, (1970).

[Sin74] I.Singer. The theory of best approximation and funtional analysis, CBMSNSF Regional Conf. Ser. in Appl. Math. 13 (1974). 